\begin{tabular}{|c|c|c|c|c|c|c|c|}
\hline \multicolumn{8}{|c|}{ Adult Samples } \\
\hline Author (Year) & $\begin{array}{l}\text { Sample, } \\
\text { Sampling Method }\end{array}$ & $\begin{array}{l}\text { Percentage of Sexual Minority } \\
\text { Individuals, Dimensions of } \\
\text { Sexual Orientation, } \\
\text { Assessment Method }\end{array}$ & Outcome Variables & $\begin{array}{l}\text { Main Results (SM vs. HET) } \\
\text { All Effects ORs if not other specified }\end{array}$ & Subgroup Differences & $\begin{array}{l}\text { Gender } \\
\text { Differences }\end{array}$ & Note \\
\hline $\begin{array}{l}\text { American } \\
\text { College Health } \\
\text { Association, } \\
\text { National } \\
\text { College Health } \\
\text { Assessment } \\
\text { (ACHA-NCHA) }\end{array}$ & $\begin{array}{l}\text { USA, young adults, } \\
\text { random samples within } \\
\text { schools or random samples of } \\
\text { classrooms }\end{array}$ & $\begin{array}{l}\text { Paper \& web-Surveys, } \\
\text { (only paper spring } 2000 \text { - fall 2002) }\end{array}$ & & & & & \\
\hline $\begin{array}{l}\text { J. Blosnich \& } \\
\text { Bossarte } \\
(2012)^{b}\end{array}$ & $\begin{array}{l}11,046 \text { adults, } 18-24 \text { years-old } \\
\text { (only } 5 \% \text { random sample of } \\
\text { HET). } \\
\text { Fall } 2008 \text { and spring } 2009 \\
\text { surveys }\end{array}$ & $\begin{array}{l}2 \% \text { identified as } L G, 3 \% \text { as } B \text {, and } \\
2 \% \text { as } Q \text { (that were removed from } \\
\text { analysis). }\end{array}$ & $\begin{array}{l}\text { SA in the last year with single } \\
\text { item }\end{array}$ & $\begin{array}{l}\text { Ss effects for LG (3.8) and B (5.4) } \\
\text { ORs created by authors, in paper adjustment for } \\
\text { mental health diagnosis }\end{array}$ & $\begin{array}{l}\text { SO difference somewhat } \\
\text { larger for } B \text { than } L G \\
\text { participants }\end{array}$ & Not reported & \\
\hline $\begin{array}{l}\text { Duryea \& } \\
\text { Frantz (2011) }\end{array}$ & $\begin{array}{l}54,111 \text { adults, } 18-24 \text { years-old } \\
\text { Spring } 2005 \text { survey }\end{array}$ & $\begin{array}{l}+++ \\
1.2 \% \text { of women identified as } L \text { and } \\
4 \% \text { as } B ; 3.2 \% \text { of men identified as } \\
G \text { and } 1.7 \% \text { as } B \text {. }\end{array}$ & $\begin{array}{l}\text { Among those with } \geq 1 \text { times } \\
\text { binge drinking in past } 2 \text { weeks; } \\
\text { scores calculated for } 9 \text { negative } \\
\text { consequences items, i.e., } \\
\text { drinking and driving, fighting, } \\
\text { unsafe sex, injuring self or other. }\end{array}$ & $\begin{array}{l}\text { SO differences for binge drinking not given. } \\
\text { Negative consequences (effects d): } \\
\text { G men: -.2, B men: } 0.2 \\
\text { L women: }-.2 \text {, B women: } 0.2\end{array}$ & $\begin{array}{l}\text { Bs had higher levels than } \\
\text { HET for negative } \\
\text { consequences from } \\
\text { drinking, } \\
\text { LG had lower levels than } \\
\text { HET. }\end{array}$ & Comparable & \\
\hline $\begin{array}{l}\text { D. L. Kerr, } \\
\text { Santurri, \& } \\
\text { Peters (2013) }\end{array}$ & $\begin{array}{l}92,410 \text { women aged } 18 \text { to } 24 \\
\text { years-old. } \\
\text { Three random subsamples } \\
\text { from fall } 2008 \text {, spring/fall } 2009 \\
\text { surveys: } 6,689 \mathrm{HET}, 849 \mathrm{~L}, \\
2,456 \mathrm{~B} \text {. }\end{array}$ & $\begin{array}{l}+++ \\
5 \% \text { identified as } B \text { or } L . \\
Q \text { and trans were removed. }\end{array}$ & $\begin{array}{l}\text { For this paper, item on feeling } \\
\text { so depressed that it was difficult } \\
\text { function (past year), } \\
\text { overwhelming anxiety (past } \\
\text { year), diagnosis of depression } \\
\text { (lifetime), and SA (past year) }\end{array}$ & $\begin{array}{l}\text { All effects ss: } \\
\text { feeling depressed (L 2.3, B 2.8) } \\
\text { feeling anxious (L 1.7, B 2.1) } \\
\text { diagnosis of depression (L 2.4, B 3.1) } \\
\text { SA (L 4.4, B 5.1) }\end{array}$ & $\begin{array}{l}\text { Slightly larger SO } \\
\text { differences for B than L } \\
\text { women. }\end{array}$ & - & \\
\hline $\begin{array}{l}\text { D. L. Kerr, } \\
\text { Ding, \& Chaya } \\
(2014)^{b}\end{array}$ & $\begin{array}{l}65,281 \text { adults, } 18-25 \text { years- } \\
\text { old, } \\
\text { fall semesters } 2009-2011 \\
\text { (three years) }\end{array}$ & $\begin{array}{l}+++ \\
5 \% \text { identified as SM }(2 \% \text { as LG } \\
\text { and } 3.1 \% \text { as B) } \\
\text { Q were excluded }\end{array}$ & $\begin{array}{l}\text { Past month and lifetime nicotine, } \\
\text { alcohol, and drug use } \\
\text { (marijuana and any other illegal } \\
\text { drug use for this analysis), and } \\
\text { consequences of alcohol use }\end{array}$ & $\begin{array}{l}\text { Past month results, all ss if not marked nss. } \\
\text { Women: tobacco ( } \mathrm{L} 2.3, \mathrm{~B} 2.9) \text {, alcohol (L } 1.0 \mathrm{nss}, \\
\text { B 1.5), marijuana ( } \mathrm{L} 2.1, \mathrm{~B} 2.8) \text {, other illegal drug } \\
\text { use ( } \mathrm{L} 1.6, \mathrm{~B} 2.9) \text {. } \\
\text { Men: tobacco (G } 1.0 \mathrm{nss}, \mathrm{B} 1.4) \text {, alcohol (G 1.3, } \\
\text { B 1.5), marijuana (G } 1.0 \mathrm{nss}, \mathrm{B} 1.7) \text {, and other } \\
\text { illegal drug use (G } 1.3 \mathrm{nss}, \mathrm{B} 1.8) \text {. } \\
\text { Results comparable for lifetime results }\end{array}$ & $\begin{array}{l}\text { Larger effects among B } \\
\text { compared to their LG } \\
\text { counterparts. }\end{array}$ & $\begin{array}{l}\text { Somewhat larger } \\
\text { effects among } \\
\text { women than men. }\end{array}$ & \\
\hline
\end{tabular}


Online supplemental material to Plöderl \& Tremblay (2015). Mental health of sexual minorities. A systematic review. International Review of Psychiatry. http://dx.doi.org/10.3109/09540261.2015.1083949

\begin{tabular}{|c|c|c|c|c|c|c|c|}
\hline $\begin{array}{l}\text { D. Kerr, Ding, } \\
\text { Burke, \& Ott- } \\
\text { Walter (2015) }\end{array}$ & $\begin{array}{l}42,986 \text { female } \\
\text { undergraduates, three fall } \\
\text { semesters (2009-2011) }\end{array}$ & $\begin{array}{l}+++ \\
1.2 \% \text { identified as } L, 3.7 \% \text { as B }\end{array}$ & $\begin{array}{l}\text { Alcohol / various drug use, past } \\
30 \text { days, responses with use on } \\
\text { one/two or more days equated } \\
\text { to "drug use." Non-prescribed } \\
\text { prescription use of various } \\
\text { drugs, past year, equated to } \\
\text { prescription drug misuse }\end{array}$ & $\begin{array}{l}\text { Alcohol: } 1.0 \mathrm{nss}, 2.5 \mathrm{nss} \\
\text { Cigarettes: } 2.4 \mathrm{ss}, 3.2 \mathrm{ss} \\
\text { Marijuana: } 2.0 \mathrm{ss}, 2.8 \mathrm{ss} \\
\text { Inhalants: } 1.2 \mathrm{~ns}, 3.0 \mathrm{ss} \\
\text { Steroids: } 3.2 \mathrm{nss}, 1.1 \mathrm{nss} \\
\text { Cocaine: } 0.9 \mathrm{nss}, 2.5 \mathrm{ss} \\
\text { Metamphetamine: } 2.7 \mathrm{nss}, 5.4 \mathrm{ss} \\
\text { Other Amphetamine: } 1.1 \mathrm{nss}, 2.2 \mathrm{ss} \\
\text { Sedatives: } 2.2 \mathrm{ss}, 3.5 \mathrm{ss} \\
\text { Hallucigenes: } 2.9 \mathrm{ss}, 6.3 \mathrm{ss} \\
\text { Opiates: } 2.2 \mathrm{nss}, 4.6 \mathrm{ss} \\
\text { MDMA-ecstasy: } 0.9 \mathrm{nss}, 4.1 \mathrm{ss} \\
\text { Other club drug: } 1.6 \mathrm{nss}, 3.2 \mathrm{ss} \\
\text { Other illegal drug: } 2.3 \mathrm{ss}, 3.1 \mathrm{ss} \\
\text { Prescription drugs (unprescribed): } \\
\text { L 1.3-1.7, most ss; B } 1.8-2.1 \text {, all ss }\end{array}$ & $\begin{array}{l}\text { Larger effects among B } \\
\text { than L women except } \\
\text { alcohol (no difference) } \\
\text { B had ss higher levels of } \\
\text { current use of all drugs } \\
\text { included in the study than } \\
\text { L, except steroids. }\end{array}$ & - & $\begin{array}{l}\text { Adjustment } \\
\text { includes } \\
\text { partnership } \\
\text { status (in } \\
\text { addition to } \\
\text { socio- } \\
\text { demographic } \\
\text { variables) }\end{array}$ \\
\hline $\begin{array}{l}\text { Lytle, De Luca, } \\
\text { \& Blosnich } \\
(2014)\end{array}$ & $\begin{array}{l}89,199 \text { adults, } 18-24 \text { years- } \\
\text { old. } \\
\text { Fall } 2008 \text { and spring } 2009 \\
\text { surveys. }\end{array}$ & $\begin{array}{l}\text { 4.2-8.3\% identified as LGB, } \\
\text { depending on race. }\end{array}$ & $\begin{array}{l}\text { Past year SA. } \\
\text { Past year diagnosis or treatment } \\
\text { for depression. }\end{array}$ & $\begin{array}{l}\text { Depression diagnosis (all ss): } \\
\text { Black: 4.0, Asian: } 3.4, \text { Latino: } 2.6 \text {, Multiracial: } 3.0 \text {, } \\
\text { Other: } 4.3 \text {, Non-Hispanic White: } 2.8 \\
\text { SA (all ss): Blacks: } 3.9 \text {, Asian: } 3.7 \text {, Latino: } 2.8 \text {, } \\
\text { Multiracial: } 5.8, \text { Other: } 4.3 \text {, Non-Hispanic White: } \\
\text { 4.2. Further analysis is available online (Ramsay } \\
\text { \& Tremblay (2015) }\end{array}$ & None reported & None reported & $\begin{array}{l}\text { Effects } \\
\text { reported within } \\
\text { racial } \\
\text { subgroups }\end{array}$ \\
\hline $\begin{array}{l}\text { Matthews- } \\
\text { Ewald, Zullig, \& } \\
\text { Ward (2014) }\end{array}$ & $\begin{array}{l}\text { 110,412 adults, mean age } \\
22.1 \text { years-old, } \\
2008-2009 \text { surveys (no } \\
\text { semesters specified) }\end{array}$ & $\begin{array}{l}\text { +++ } \\
\text { Identification as LGBQ } \\
\text { (transgender individuals excluded) }\end{array}$ & $\begin{array}{l}\text { Outcome variable: past year } \\
\text { diagnoses or treatment for } \\
\text { anorexia or bulimia by } \\
\text { professional (yes/no). }\end{array}$ & $\begin{array}{l}\text { Ss effects among men (G: } 3.9, \mathrm{~B}: 4.2, \mathrm{Q}: 4.5) \text {, nss } \\
\text { effects among women (L: } 1.0, \mathrm{~B} 1.3, \mathrm{Q} 1.1) \text {. } \\
\text { CAVE: Controls: Cigarette use past month, binge } \\
\text { drinking ( } \geq 5 \text { drinks in a row) in past two weeks } \\
\text { (yes/no), depressive symptoms in past year }\end{array}$ & $\begin{array}{l}\text { Comparable effects for } \\
\text { LG, B, Q. }\end{array}$ & $\begin{array}{l}\text { Larger effects for } \\
\text { men than women. }\end{array}$ & $\begin{array}{l}\text { ORs adjusted } \\
\text { for drinking, } \\
\text { depression etc: } \\
\text { likely under- } \\
\text { estimations! }\end{array}$ \\
\hline $\begin{array}{l}\text { Oswalt \& Wyatt } \\
(2011)^{b}\end{array}$ & $\begin{array}{l}27,454 \text { adults, } 18-49 \text { years- } \\
\text { old, } 84.5 \% \text { between } 18-24 \\
\text { years. } \\
\text { Fall } 2009 \text { survey }\end{array}$ & $\begin{array}{l}\text { 7\% identified as LGBQ (LG 1.9\%, } \\
\text { B 2.9\%, Q } 1.5 \%) \\
\text { Transgender }(0.1 \%) \text { excluded }\end{array}$ & $\begin{array}{l}\text { Frequency of having felt sad, } \\
\text { depressed, anxiety (and more) } \\
\text { in past two weeks, past month, } \\
\text { past year, combined. } \\
\text { Past year diagnosis or treatment } \\
\text { of mental disorder (depression, } \\
\text { anxiety, panic attacks, plus } \\
\text { others). } \\
\text { SA (past year and before) }\end{array}$ & $\begin{array}{l}\text { Past year effects: } \\
\text { Felt sad } L G / B / Q: 1.7,2.0,1.8 \\
\text { Depressed } L G / B / Q: 2.1,2.6,2.3 \\
\text { Anxiety } L G / B / Q: \text { : } 2.0,2.0,1.6 \\
\text { Diagnosis past year: } \\
\text { Depression } L G / B / Q: 2.4,3.1,2.4 \\
\text { Anxiety } L G / B / Q: 2.1,2.5,1.9 \\
\text { Panic Attack } L G / B / Q: 0.25,2.6,2.3 \\
\text { SA past year: } L G / B / Q: 4.6,3.7,2.7 \\
\text { SA lifetime: } L G / B / Q ; 3.1,4.8,2.2\end{array}$ & $\begin{array}{l}\text { Comparable effects } \\
\text { between } L G \text { and } B \text {, } \\
\text { somewhat smaller effects } \\
\text { among } Q \text { than } L G B \text {. }\end{array}$ & None reported & $\begin{array}{l}\text { Results for SA } \\
\text { see Ramsay \& } \\
\text { Tremblay } \\
(2015)\end{array}$ \\
\hline $\begin{array}{l}\text { Pelts \& Albright } \\
\text { (2014) }\end{array}$ & $\begin{array}{l}702 \text { veterans, making } 3 \% \text { of } \\
\text { the } 2011 \text { fall study. }\end{array}$ & $7 \%$ identified as $L G B Q, n=45$ & $\begin{array}{l}\text { Past year diagnosis or treatment } \\
\text { of mental disorder (ADHD, }\end{array}$ & $\begin{array}{l}\text { Diagnosis/treatment (past year): } \\
\text { Depression: } 4.0 \mathrm{ss} \text {, Bipolar } 4.2 \mathrm{ss}\end{array}$ & None reported & None reported & \\
\hline
\end{tabular}


Online supplemental material to Plöderl \& Tremblay (2015). Mental health of sexual minorities. A systematic review. International Review of Psychiatry. http://dx.doi.org/10.3109/09540261.2015.1083949




Online supplemental material to Plöderl \& Tremblay (2015). Mental health of sexual minorities. A systematic review. International Review of Psychiatry. http://dx.doi.org/10.3109/09540261.2015.1083949

\begin{tabular}{|c|c|c|c|c|c|c|c|}
\hline & & & & $\begin{array}{l}\text { Anxiety Diagnosis: 2.1, 4.3, 2.9 } \\
\text { Self-harm past } 6 \text { months: 4.3, 8.0, } 2.1\end{array}$ & & & \\
\hline $\begin{array}{l}\text { Behavioral } \\
\text { Risk Factor } \\
\text { Surveillance } \\
\text { Study } \\
\text { (BRFSS) }\end{array}$ & $\begin{array}{l}\text { Probability based complex } \\
\text { sampling design, USA, } \\
\text { stratified random sample, } \\
\text { ( } \geq 18 \text { years-old) }\end{array}$ & $\begin{array}{l}\text { Computer assisted telephone } \\
\text { interviews }\end{array}$ & & & & & \\
\hline $\begin{array}{l}\text { Balsam, } \\
\text { Beadnell, \& } \\
\text { Riggs (2012) }\end{array}$ & $\begin{array}{l}\text { 103,087 adults, mean age } \\
\text { n.a., } \\
\text { Washington, 2003-2007 }\end{array}$ & $\begin{array}{l}+++ \\
3 \% \text { of women }(1.4 \% \mathrm{~L} \text { and } 1.6 \% \\
\text { B) and } 2.8 \% \text { of men }(1.9 \% \mathrm{G} \text { and } \\
0.9 \% \mathrm{~B}) \text { identified as LGB. }\end{array}$ & $\begin{array}{l}\text { Alcohol use, smoking status, } \\
\text { poor mental health ( } 21-30 \text { days } \\
\text { of past } 30 \text { days vs. less days } \\
\text { poor mental health) }\end{array}$ & \begin{tabular}{|l} 
Ss differences for all SM subgroups: \\
Men (G, B) \\
Binge drinking: $1.6,2.0$, Heavy drinking: $1.5,2.4$ \\
Smoking: $2.1,2.8$, Poor mental health: $1.8,3.4$ \\
$\quad$ Women (L, B) \\
Binge drinking: $1.5,3.6$, Heavy drinking: $1.7,2.5$ \\
Smoking: $2.2,3.3$, Poor mental health: $1.6,3.3$
\end{tabular} & $\begin{array}{l}\text { Larger SO differences for } \\
\text { bisexual compared to LG } \\
\text { men and women. } \\
\text { See also Fredricksen } \\
\text { (2013) for comparison of } L \\
\text { and B women }\end{array}$ & $\begin{array}{l}\text { Comparable SO } \\
\text { differences }\end{array}$ & \\
\hline $\begin{array}{l}\text { J. R. Blosnich, } \\
\text { Bossarte, \& } \\
\text { Silenzio (2012) }\end{array}$ & $\begin{array}{l}1,700 \text { adult veterans, } \\
\text { mean age n.a., Washington, } \\
\text { surveys } 2005-2010\end{array}$ & 3.6\% identified as LGB & $\begin{array}{l}\text { Poor mental health }>15 \text { days of } \\
\text { past } 30 \text { days }\end{array}$ & Nss effect (1.6) & None reported & None reported & \\
\hline $\begin{array}{l}\text { J. Blosnich, } \\
\text { Foynes, \& } \\
\text { Shipherd } \\
\text { (2013) }\end{array}$ & $\begin{array}{l}1,908 \text { adult female veterans, } \\
\text { mean-age n.a., surveys from } \\
10 \text { states, } 2010\end{array}$ & $5.9 \%$ identified as LB, & $\begin{array}{l}\text { Frequent mental distress ( } \geq 6 \\
\text { days in past month), smoking }\end{array}$ & $\begin{array}{l}\text { Frequent mental distress } 3.0 \\
\text { Current smoking } 2.3\end{array}$ & None reported & - & $\begin{array}{l}\text { Veteran LB } \\
\text { more at risk } \\
\text { than non- } \\
\text { veteran LB. }\end{array}$ \\
\hline $\begin{array}{l}\text { J. Blosnich, } \\
\text { Bossarte, } \\
\text { Silver, \& } \\
\text { Silenzio (2013) }\end{array}$ & $\begin{array}{l}\text { 13,927 partnered adult } \\
\text { veterans, mean 36.5-47.2 } \\
\text { years-old, depending on } \\
\text { subgroup. } \\
\text { Survey } 2004\end{array}$ & $\begin{array}{l}0.7 \% \text { indicated having a same-sex } \\
\text { partner }\end{array}$ & $\begin{array}{l}\text { Frequent mental distress ( } \geq 6 \\
\text { days in past month), smoking, } \\
\text { past month binge drinking }\end{array}$ & $\begin{array}{l}\text { All effects nss. } \\
\text { Frequent mental distress } 1.3 \\
\text { Smoking } 1.4 \\
\text { Binge drinking } 1.5\end{array}$ & & & $\begin{array}{l}\text { Among SM, } \\
\text { veterans only } \\
\text { slightly more at } \\
\text { risk than non- } \\
\text { veterans, few } \\
\text { SM individuals }\end{array}$ \\
\hline $\begin{array}{l}\text { J. R. Blosnich } \\
\text { \& Silenzio } \\
\text { (2013) }\end{array}$ & $\begin{array}{l}11,665 \text { adult veterans, mean } \\
\text { age > } 60 \text { years-old, survey } \\
\text { from } 10 \text { different states }\end{array}$ & 2.0\% identified as LGB, & $\begin{array}{l}\text { Smoking, binge drinking past } \\
\text { month }\end{array}$ & $\begin{array}{l}\text { Smoking: } 1.3, \text { nss } \\
\text { Binge drinking: } 0.8, \text { nss }\end{array}$ & None reported & None reported & \\
\hline $\begin{array}{l}\text { J. R. Blosnich, } \\
\text { Farmer, Lee, } \\
\text { Silenzio, \& } \\
\text { Bowen (2014) }\end{array}$ & $\begin{array}{l}\text { 93,414 adults, mean age } 35.1- \\
47.3 \text {, depending on subgroup, } \\
\text { USA, } \\
\text { Survey from } 10 \text { states }\end{array}$ & $\begin{array}{l}+++ \\
2.4 \% \text { identified as LGB (35\% of } \\
\text { these as B), other/don't know and } \\
\text { those who refused were excluded }\end{array}$ & $\begin{array}{l}\text { Frequent mental distress ( } \geq 6 \\
\text { days in past month), } \\
\text { smoking, past month binge } \\
\text { drinking }\end{array}$ & $\begin{array}{l}\text { Women } \\
\text { Mental distress B } 1.5 \text { nss, L n.a., smoking: B } 2.1 \\
\text { ss, L } 1.9 \text { ss, binge drinking: B } 1.7 \text { ss, L } 1.6 \text { ss. } \\
\quad \text { Men } \\
\text { Mental distress: B } 2.9 \mathrm{ss}, \mathrm{G} 1.9 \mathrm{ss} \text {, smoking: B } \\
\text { and G } 1.9 \mathrm{ss} \text {, binge drinking: B 1.4, G } 1.2\end{array}$ & $\begin{array}{l}\text { Somewhat larger SO } \\
\text { difference for B men and } \\
\text { women compared their LG } \\
\text { counterparts }\end{array}$ & $\begin{array}{l}\text { Comparable SO } \\
\text { differences }\end{array}$ & \\
\hline J. R. Blosnich & 20,060 adults, mean age 39.7 & $2.7 \%$ self-identified as LGB & Frequent mental distress: & Ss effect, 1.7 & None reported & None reported & \\
\hline
\end{tabular}


Online supplemental material to Plöderl \& Tremblay (2015). Mental health of sexual minorities. A systematic review. International Review of Psychiatry. http://dx.doi.org/10.3109/09540261.2015.1083949

\begin{tabular}{|c|c|c|c|c|c|c|}
\hline $\begin{array}{l}\text { \& Andersen } \\
(2015)\end{array}$ & $\begin{array}{l}\text { (SM) and } 46.7 \text { (HET) years- } \\
\text { old, survey } 2010 \text { from Maine, } \\
\text { Washington, and Wisconsin. }\end{array}$ & & $\begin{array}{l}\geq 14 \text { days self-reported poor } \\
\text { mental health in past month } \\
\text { (yes/no). }\end{array}$ & Adjusted for demographic controls & & \\
\hline $\begin{array}{l}\text { Conron, } \\
\text { Mimiaga, \& } \\
\text { Landers } \\
\text { (2010), b } \\
\text { see also } \\
\text { Landers, } \\
\text { Mimiaga, \& } \\
\text { Conron (2011) } \\
\text { for overall } \\
\text { effects }\end{array}$ & $\begin{array}{l}\text { 67,359 adults, } 18-64 \text { years- } \\
\text { old, Massachusetts surveys } \\
2001-2008\end{array}$ & $\begin{array}{l}+++ \\
2 \% \text { identified as } L G, 1 \% \text { as B. } \\
Q(0.5 \%) \text { and "other " }(0.5 \%) \text { were } \\
\text { excluded }\end{array}$ & $\begin{array}{l}\text { Feeling sad/blue } \geq 15 \text { days in } \\
\text { past month, current smoking, } \\
\text { binge drinking and illicit drug use } \\
\text { past month }\end{array}$ & \begin{tabular}{|l} 
Women (L, B) \\
Feeling sad/blue: $1.0 \mathrm{nss}, 2.4 \mathrm{ss}$ \\
Current smoking: $1.9 \mathrm{ss}, 1.6 \mathrm{ss}$ \\
Binge drinking: $1.4 \mathrm{nss}, 1.5 \mathrm{ss}$ \\
Illicit drug use: $2.1 \mathrm{ss}, 9.1 \mathrm{ss}$ \\
\multicolumn{1}{c}{ Men (G, B) } \\
Feeling sad/blue: $1.5 \mathrm{nss}, 2.1 \mathrm{nss}$ \\
Current smoking: $2.4 \mathrm{ss}, 2.0 \mathrm{ss}$ \\
Binge drinking: $1.1 \mathrm{nss}, 0.8 \mathrm{nss}$ \\
Illicit drug use: $3.1 \mathrm{ss}, 2.3 \mathrm{ss}$
\end{tabular} & $\begin{array}{l}\text { B men and women had } \\
\text { higher OR for mental } \\
\text { distress than } L G \text { but were } \\
\text { comparable for smoking } \\
\text { and binge drinking. }\end{array}$ & $\begin{array}{l}\text { B women had } \\
\text { larger SO } \\
\text { differences than } B \\
\text { men but } L \text { women } \\
\text { were comparable } \\
\text { to } G \text { men. }\end{array}$ \\
\hline $\begin{array}{l}\text { Dilley, } \\
\text { Simmons, } \\
\text { Boysun, } \\
\text { Pizacani, \& } \\
\text { Stark (2010); b } \\
\text { also Balsam, et } \\
\text { al. (2012) }\end{array}$ & $\begin{array}{l}\text { 79,500 adults, mean age n.a., } \\
\text { Washington 2003-2006. }\end{array}$ & $\begin{array}{l}+++ \\
2.4 \% \text { of women }(1.2 \% \mathrm{~L} \text { and } 1.2 \% \\
\text { B) and } 2.4 \% \text { of men }(1.6 \% \mathrm{G} \text { and } \\
0.8 \% \mathrm{~B}) \text { identified as } \mathrm{LGB}\end{array}$ & $\begin{array}{l}\text { Heavy drinking ( } \geq 2 \text { drinks per } \\
\text { day in past month), current } \\
\text { smoking, poor mental health ( } \geq \\
10 \text { days of past } 30 \text { days vs. less } \\
\text { days poor mental health) }\end{array}$ & $\begin{array}{l}\text { Smoking : L 2.4, B women 2.5, G 2.2, B men 2.3, } \\
\text { all ss. } \\
\text { Heavy drinking: L } 1.8 \text { ss, B women } 2.9 \text { ss, G } 1.2 \\
\text { nss, B men } 2.0 \text { nss). } \\
\text { Poor mental health: L 1.7, B women } 3.1, \text { G } 2.3 \text {, } \\
\text { B men 2.1, all ss }\end{array}$ & $\begin{array}{l}\text { Larger effects for B for } \\
\text { heavy drinking but not for } \\
\text { smoking and poor mental } \\
\text { health }\end{array}$ & $\begin{array}{l}\text { Comparable but } \\
\text { somewhat larger } \\
\text { effect among } \\
\text { women for heavy } \\
\text { drinking. }\end{array}$ \\
\hline $\begin{array}{l}\text { Fredriksen- } \\
\text { Goldsen, Kim, } \\
\text { Barkan, } \\
\text { Muraco, \& } \\
\text { Hoy-Ellis } \\
(2013)^{\mathrm{b}}\end{array}$ & $\begin{array}{l}96,992 \text { older adults }(\geq 50 \\
\text { years), } \\
\text { Washington 2003-2010, }\end{array}$ & $\begin{array}{l}1.5 \% \text { of women }(1.0 \% \mathrm{~L} \text { and } 0.6 \% \\
\text { B) and } 1.9 \% \text { of men }(1.3 \% \mathrm{G} \text { and } \\
0.5 \% \mathrm{~B}) \text { identified as } \mathrm{LGB} \text {. }\end{array}$ & $\begin{array}{l}\text { Excessive drinking, i.e., } \geq 4 \\
\text { drinks ( } \geq 5 \text { for men) in a row in } \\
\text { past month, smoking status, } \\
\text { poor mental health ( } \geq 14 \text { days of } \\
\text { past } 30 \text { days vs. less days poor } \\
\text { mental health) }\end{array}$ & $\begin{array}{l}\text { All effects ss (women, men) } \\
\text { Smoking: } 1.6,1.7 \\
\text { Excessive drinking : } 1.4,1.7 \\
\text { Poor mental health: } 1.4,1.8\end{array}$ & None reported & Comparable \\
\hline $\begin{array}{l}\text { Matthews \& } \\
\text { Lee (2014) b }\end{array}$ & $\begin{array}{l}\text { 9,876 adults, mean age n.a., } \\
\text { North Carolina }\end{array}$ & $\begin{array}{l}2.3 \% \text { of men ( } G \text { and } B \text { together) } \\
\text { and } 1.8 \% \text { of women ( } L \text { and B } \\
\text { together) identified as } L G B\end{array}$ & $\begin{array}{l}\text { Poor mental health in past } \\
\text { month, depression told by } \\
\text { doctor, current smoking, past } \\
\text { month binge drinking ( } \geq 4 \text { drinks } \\
\text { women, } \geq 5 \text { men) in a row }\end{array}$ & $\begin{array}{l}\text { Poor mental health } 2.6 \mathrm{ss} \text { (men), } 2.6 \mathrm{ss} \text { (women) } \\
\text { Depression } 3.6 \mathrm{ss}, 3.0 \mathrm{ss} \\
\text { Smoking } 0.44 \mathrm{nss}, 2.0 \mathrm{ss} \\
\text { Binge drinking } 0.50 \mathrm{nss}, 1.6 \mathrm{nss}\end{array}$ & Not reported & $\begin{array}{l}\text { Comparable for } \\
\text { depression and } \\
\text { poor mental } \\
\text { health. Larger } \\
\text { effect for women } \\
\text { for substance use }\end{array}$ \\
\hline $\begin{array}{l}\text { Rothman, } \\
\text { Sullivan, } \\
\text { Keyes, \& } \\
\text { Boehmer } \\
(2012)^{b}\end{array}$ & $\begin{array}{l}\text { 5,658 adults, } 18-60 \text { years-old, } \\
\text { Massachusetts, } 2002\end{array}$ & 3.1\% identified as LGB & $\begin{array}{l}\text { Frequent poor mental health and } \\
\text { depression ( } \geq 15 \text { days in past } \\
\text { month), any past month } \\
\text { smoking, binge drinking in past } \\
\text { month, illicit drug use (past } \\
\text { month, lifetime) }\end{array}$ & $\begin{array}{l}\text { Lifetime illicit drug use: } 3.5 \mathrm{ss} \text {, } \\
\text { past month illicit drug use } 3.0 \mathrm{ss} \text {, } \\
\text { binge drinking } 1.6 \mathrm{nss}, \\
\text { smoking } 3.1 \mathrm{ss} \text {, } \\
\text { poor mental health } 1.4, \mathrm{nss}, \\
\text { depression } 2.4, \mathrm{ss} \text {. }\end{array}$ & None reported & None reported \\
\hline
\end{tabular}


Online supplemental material to Plöderl \& Tremblay (2015). Mental health of sexual minorities. A systematic review. International Review of Psychiatry. http://dx.doi.org/10.3109/09540261.2015.1083949

\begin{tabular}{|c|c|c|c|c|c|c|c|}
\hline $\begin{array}{l}\text { Canadian } \\
\text { Communiy } \\
\text { Health Survey } \\
2003\end{array}$ & $\begin{array}{l}\text { Representative sample of the } \\
\text { Canadian population }\end{array}$ & & & & & & \\
\hline $\begin{array}{l}\text { Brennan, Ross, } \\
\text { Dobinson, } \\
\text { Veldhuizen, \& } \\
\text { Steele (2010) }\end{array}$ & $\begin{array}{l}49,901 \text { adults, } 18-59 \text { years- } \\
\text { old, } \\
\text { survey } 2003\end{array}$ & $\begin{array}{l}+++ \\
1.3 \% \\
\text { identified as } L G, 0.6 \% \text { as B }\end{array}$ & $\begin{array}{l}\text { Presence of mood or anxiety } \\
\text { disorders, poorer perceived } \\
\text { mental health, daily smoking } \\
\text { (yes/no), risky drinking (> } 8 \\
\text { drinks per week) }\end{array}$ & $\begin{array}{l}\text { Mood/anxiety disorder (all ss): G 3.1, B } 2.4 \\
\text { Poor mental health (all nss): G 1.6, B: } 1.5 \\
\text { Daily smoking: G and B 1.2, nss. } \\
\text { Risky drinking: G } 0.7, \text { B 1.3, nss. }\end{array}$ & $\begin{array}{l}\text { For mood/anxiety larger } \\
\text { effects for } \mathrm{G} \text { than } \mathrm{B} \text {, but } \\
\text { reversed for risky drinking, } \\
\text { comparable for poor mental } \\
\text { health or smoking. }\end{array}$ & - & \\
\hline $\begin{array}{l}\text { Pakula \& } \\
\text { Shoveller } \\
(2013)^{b}\end{array}$ & $\begin{array}{l}\text { 76,630 adults, } \\
18-59 \text { years-old, } \\
2007-2008\end{array}$ & $\begin{array}{l}2.72 \% \text { identified as LGB. } \\
\text { Computer assisted in person and } \\
\text { telephone interview }\end{array}$ & $\begin{array}{l}\text { Self reported mood } \\
\text { disorder diagnosis (diagnosed } \\
\text { by a professional) }\end{array}$ & $\begin{array}{l}\text { Mood disorder ( } 2.8, \mathrm{ss}) \\
\text { In adjusted model (age, education, regular GP, } \\
\text { community belonging, life stress AOR } 3.5 \text { among } \\
\text { men and } 2.6 \text { among women (all ss). }\end{array}$ & None reported & $\begin{array}{l}\text { Somewhat larger } \\
\text { effect for males in } \\
\text { the adjusted } \\
\text { model }\end{array}$ & \\
\hline $\begin{array}{l}\text { Steele, Ross, } \\
\text { Dobinson, } \\
\text { Veldhuizen, \& } \\
\text { Tinmouth } \\
\text { (2009) }\end{array}$ & $\begin{array}{l}61,713 \text { adult women, } 18-59 \\
\text { years-old, } \\
2003\end{array}$ & $\begin{array}{l}+++ \\
1.6 \% \text { identified as SM }(0.7 \% \text { as } \\
\text { HO, } 0.9 \% \text { as B), labels were } \\
\text { explained with sexual relations, } \\
\text { e.g., "bisexual" (sexual relations } \\
\text { with people of both sexes). }\end{array}$ & $\begin{array}{l}\text { Mood or anxiety disorder } \\
\text { diagnosed by health } \\
\text { professional, perceived mental } \\
\text { health (single item), } \\
\text { smoking (daily vs. not daily), } \\
\text { drinking (> } 8 \text { drinks a week) }\end{array}$ & $\begin{array}{l}\text { Mood/anxiety disorder: HO } 1.4 \mathrm{ss,} \mathrm{B} 4.2 \mathrm{ss.} \\
\text { Poor mental health: } \mathrm{HO} 1.2 \mathrm{nss}, \mathrm{B} 4.4 \mathrm{ss} \\
\text { Smoking: HO 1.9, B 2.4, all ss. } \\
\text { Drinking: HO 2.6, B 2.3, all ss. }\end{array}$ & $\begin{array}{l}\text { B larger effect than } \mathrm{HO} \text { for } \\
\text { mental health but less so } \\
\text { for substance use }\end{array}$ & - & $\begin{array}{l}\text { Few SM } \\
\text { women in } \\
\text { sample. }\end{array}$ \\
\hline $\begin{array}{l}\text { Tjepkema } \\
(2008)^{\mathrm{b}}\end{array}$ & $\begin{array}{l}159,824 \text { adults, } 18-59 \text { years- } \\
\text { old, } \\
\text { surveys } 2003 \text { and } 2005\end{array}$ & $\begin{array}{l}+++ \\
1.1 \% \text { identified as LG, } 0.8 \% \text { as B. } \\
\text { See Steele at al. (2009) }\end{array}$ & $\begin{array}{l}\text { Mood or anxiety disorder } \\
\text { diagnosed by health } \\
\text { professional, perceived mental } \\
\text { health (single item), } \\
\text { disability in past } 2 \text { weeks due to } \\
\text { mental health or alcohol. }\end{array}$ & $\begin{array}{l}\text { All effects ss if not noted otherwise } \\
\text { Self-perceived fair to poor mental health: } \\
\text { Men, G, B: } 1.3 \text { nss, } 2.3 \text {, Women, L, B: } 1.3 \text { nss, } 3.7 \\
\text { Mood Disorder } \\
\text { Men, G, B: 3.0, 3.1; Women, L, B: } 1.5,4.0 \\
\text { Anxiety Disorder } \\
\text { Men, G, B: } 3.0,3.6 \text {; Women, L, B: } 1.5,3.5 . \\
\text { Mental Disability Day, past } 2 \text { weeks, } \\
\text { Men, G, B: } 2.5,4.8 \text {, Women: L, B: } 1.9 \text { nss, } 3.5\end{array}$ & $\begin{array}{l}\text { Larger effects among B, } \\
\text { especially among women. }\end{array}$ & Comparable & $\begin{array}{l}\text { Few SM in } \\
\text { sample }\end{array}$ \\
\hline $\begin{array}{l}\text { California } \\
\text { Quality of Life } \\
\text { Survey I } \\
\text { California } \\
\text { Health } \\
\text { Interview }\end{array}$ & $\begin{array}{l}\text { Adults, USA. } \\
\text { Random sample, complex } \\
\text { sampling design, } \\
\text { Telephone interview }\end{array}$ & & & & & & \\
\hline $\begin{array}{l}\text { J. R. Blosnich, } \\
\text { Mays, \& } \\
\text { Cochran } \\
(2014)\end{array}$ & $\begin{array}{l}444 \text { adult veterans, mean age } \\
52 \text { years-old, surveys } \\
2008 / 2009 \text { and 2012/2013 }\end{array}$ & $29 \%$ identified as LGB & $\begin{array}{l}\text { Lifetime SA from the WHO } \\
\text { Diagnostic Interview for } \\
\text { Suicidality }\end{array}$ & $\begin{array}{l}\text { SA: } 3.0, \text { ss; } \\
\text { Unadjusted: } 2.2, \text { ss } \\
\text { (In paper Relationship used for adjusted OR) }\end{array}$ & Not reported & Not reported & \\
\hline
\end{tabular}


Online supplemental material to Plöderl \& Tremblay (2015). Mental health of sexual minorities. A systematic review. International Review of Psychiatry. http://dx.doi.org/10.3109/09540261.2015.1083949

7

\begin{tabular}{|c|c|c|c|c|c|c|c|}
\hline $\begin{array}{l}\text { Boehmer, } \\
\text { Miao, \& } \\
\text { Ozonoff (2012) }\end{array}$ & $\begin{array}{l}\text { 10,942 adult cancer survivors, } \\
20-85 \text { years-old (mean ca. } \\
50 \text { ), California health interview } \\
\text { survey, 2001, 2003, 2005 }\end{array}$ & $\begin{array}{l}+++ \\
3.5 \% \text { identified as LGB (34\% of } \\
\text { these as B), }\end{array}$ & $\begin{array}{l}\text { Smoking (only current smoking } \\
\text { reported here) and amount of } \\
\text { alcohol in past month }\end{array}$ & $\begin{array}{l}\text { Any alcohol past month, all nss. } \\
\text { Women: L 1.3, B 1.1, Men: G 0.9, B } 1.2 \\
\text { Smoking: Women: L } 1.3 \text { nss, B } 2.2 \text { ss, } \\
\text { Men: G } 1.5 \text { nss, B } 2.5 \text { ss. }\end{array}$ & $\begin{array}{l}\text { Slightly elevated SO } \\
\text { differences for smoking } \\
\text { among B individuals }\end{array}$ & $\begin{array}{l}\text { Comparable } \\
\text { effects }\end{array}$ & \\
\hline $\begin{array}{l}\text { Boehmer, } \\
\text { Miao, } \\
\text { Linkletter, \& } \\
\text { Clark (2012) }\end{array}$ & $\begin{array}{l}163,221 \text { adults, USA, 20-85 } \\
\text { years-old (mean ca. 50), } \\
\text { California health interview } \\
\text { surveys 2001, 2003, 2005, } \\
2007\end{array}$ & $\begin{array}{l}+++ \\
3.4 \% \text { identified as LGB }(40 \% \text { of } \\
\text { these as B) }\end{array}$ & $\begin{array}{l}\text { Smoking (only current smoking } \\
\text { reported here), amount of } \\
\text { alcohol in past month }\end{array}$ & $\begin{array}{l}\text { Women (all ss). } \\
\text { Smoking: L 2.7, B 3.5, Alcohol: L 2.3, B 2.0s, } \\
\text { Binge drinking: L 1.9, B 2.1. } \\
\text { Men: } \\
\text { Smoking: G } 1.8 \text { ss, B } 1.2 \text { nss, Alcohol: G } 3.2 \text { ss, } \\
\text { B } 0.9 \text { nss, Binge drinking: G 1.3, B 0.8, all nss. }\end{array}$ & $\begin{array}{l}\text { Among women, } \\
\text { comparable SO differences } \\
\text { between } L \text { and } B \text {. } \\
\text { Among men, } G \text { men had } \\
\text { somewhat larger } S O \\
\text { differences than } B \text { men. }\end{array}$ & $\begin{array}{l}\text { Men had } \\
\text { somewhat smaller } \\
\text { SO differences } \\
\text { than women. }\end{array}$ & $\begin{array}{l}\text { OR from } \\
\text { regression } \\
\text { model } 1 \text { was } \\
\text { used } \\
\text { (controlling for } \\
\text { age) }\end{array}$ \\
\hline $\begin{array}{l}\text { Cochran \& } \\
\text { Mays (2009), }, \\
\text { same study as } \\
\text { Grella, } \\
\text { Cochran, } \\
\text { Greenwell, \& } \\
\text { Mays (2011) }\end{array}$ & $\begin{array}{l}\text { 2,272 adults, } 18-72 \text { years old } \\
\text { (mean } 39.2-42.5 \text { depending } \\
\text { on subgroups), from the } \\
\text { California Quality of Life } \\
\text { survey 2004/2005 }\end{array}$ & $\begin{array}{l}+++ \\
12 \% \text { were classified as SM, i.e., } \\
\text { identified as LGB }(9.5 \%) \text { or } \\
\text { heterosexual identified but with } \\
\text { homosexual experiences (HetHo, } \\
2.2 \%) \text {. }\end{array}$ & \begin{tabular}{|l|} 
Past year DSM-IV disorders \\
(MD, GAD, Panic attack, AD, \\
DD, any disorder) with CIDI-SF, \\
past month psychological \\
distress with Kesslers \\
Psychological Distress scale \\
(cutoff $\geq 15$ ).
\end{tabular} & \begin{tabular}{l} 
Adjusted RR (Table 4) \\
\multicolumn{1}{c}{ Men (G, B, HetHo) } \\
MD: $2.2 \mathrm{ss}, 1.5 \mathrm{nss}, 3.6 \mathrm{ss}$, \\
GAD: $2.3 \mathrm{ss}, 2.2 \mathrm{ss}, 2.5 \mathrm{ss}$ \\
Panic attack: $2.7 \mathrm{ss}, 1.2 \mathrm{nss}, 1.9 \mathrm{nss}$ \\
AD: $1.7 \mathrm{nss}, 1.9 \mathrm{nss}, 5.7 \mathrm{ss}$ \\
DD: $1.9 \mathrm{nss}, 1.6 \mathrm{nss}, 5.7 \mathrm{nss}$ \\
$\geq 1$ disorder: $1.9,1.7,3.3$, all ss \\
Distress: $2.0 \mathrm{ss}, 2.1 \mathrm{ss}, 2.8 \mathrm{ss}$ \\
\multicolumn{1}{c}{ Women: (L, B, HetHo) } \\
MD: $1.6 \mathrm{ss}, 1,8 \mathrm{ss}, 1.0 \mathrm{nss}$ \\
GAD: $1.2 \mathrm{nss}, 2.5 \mathrm{ss}, 1.3 \mathrm{nss}$ \\
Panik attack: $1.0 \mathrm{ss}, 2.4 \mathrm{ss}, 1.5 \mathrm{nss}$ \\
AD: $1.7 \mathrm{nss}, 1.4 \mathrm{ss}, 4.1 \mathrm{ss}$ \\
DD: $1.7 \mathrm{nss}, 2.4 \mathrm{ss}, 5.0 \mathrm{ss}$ \\
$\geq 1$ disorder: $1.1 \mathrm{nss}, 1.9 \mathrm{ss}, 1.4 \mathrm{nss}$ \\
Distress: $1.7 \mathrm{ss}, 3.1 \mathrm{ss}, 1.2 \mathrm{nss}$
\end{tabular} & $\begin{array}{l}\text { Among men, HetHo had } \\
\text { the largest SO differences, } \\
\text { B and G were comparable. } \\
\text { Among Women, B had } \\
\text { somewhat elevated SO } \\
\text { differences. } \\
\text { See Grella et al. (2011) for } \\
\text { overall effects. }\end{array}$ & $\begin{array}{l}\text { Overall there } \\
\text { were larger } \\
\text { effects for men } \\
\text { than women. } \\
G \text { men had } \\
\text { somewhat larger } \\
\text { effects than L } \\
\text { women, same } \\
\text { was true for } \\
\text { HoHet, but } \\
\text { reversed effect } \\
\text { among B. }\end{array}$ & \\
\hline $\begin{array}{l}\text { Cochran, } \\
\text { Grella, \& Mays } \\
(2012)^{\mathrm{b}}\end{array}$ & $\begin{array}{l}2,671 \text { adults, mean age } 46.5 \\
\text { years, } \\
\text { California Quality of Life } \\
\text { Survey } 2007\end{array}$ & $\begin{array}{l}4.5 \% \text { were classified as SM } \\
\text { because of same-sex sexual } \\
\text { partners since age } 18 \text { or in past } \\
\text { year }(2.1 \%) \text { or if they considered } \\
\text { themselves as LGB }(2.4 \%)\end{array}$ & $\begin{array}{l}\text { Drug and alcohol use, weekly } \\
\text { binge drinking, drug use in past } \\
\text { year }\end{array}$ & $\begin{array}{l}\text { Marijuana use: } 3.2, \mathrm{ss} \\
\text { Other illicit drug use: } 2.4 \mathrm{ss} \\
\text { Binge drinking: } 1.7, \mathrm{ss}\end{array}$ & None reported & None reported & \\
\hline $\begin{array}{l}\text { Grella et al, } \\
\text { (2001), (same } \\
\text { study as } \\
\text { Cochran \& } \\
\text { Mays, 2009) }\end{array}$ & $\begin{array}{l}\text { 2,079 adults from the } \\
\text { California Quality of Life } \\
\text { survey, 2004/2005 }\end{array}$ & $\begin{array}{l}13.8 \% \text { (unweighted) and } 5.2 \% \\
\text { (weighted) of men and } 15.3 \% \\
\text { (unweighted) and } 7.0 \% \text { (weighted) } \\
\text { of women were classified as SM, } \\
\text { i.e., identified as LGB or were } \\
\text { heterosexual identified but with } \\
\text { homosexual experiences. }\end{array}$ & $\begin{array}{l}\text { See Cochran \& Mays (2009), for } \\
\text { this analysis MD, panic attacks, } \\
\text { PTSD, and GAD were combined } \\
\text { as well as AD with DD. }\end{array}$ & $\begin{array}{l}\text { Mental health disorders: } \\
\text { Men } 2.2 \mathrm{ss} \text {, Women } 2.2 \mathrm{ss} \\
\text { AD/DD: } \\
\text { Men 1.8, Women 6.6. }\end{array}$ & None reported & $\begin{array}{l}\text { Larger effect for } \\
\text { AD/DD among } \\
\text { women. }\end{array}$ & $\begin{array}{l}\text { Discrepancies } \\
\text { between } \\
\text { percentage of } \\
\text { SM in text and } \\
\text { in Table } 1 \\
\text { where }>20 \% \\
\text { were SM. }\end{array}$ \\
\hline
\end{tabular}


Online supplemental material to Plöderl \& Tremblay (2015). Mental health of sexual minorities. A systematic review. International Review of Psychiatry. http://dx.doi.org/10.3109/09540261.2015.1083949

\begin{tabular}{|c|c|c|c|c|c|c|c|}
\hline $\begin{array}{l}\text { Wight, Leblanc, } \\
\text { \& Lee Badgett } \\
\text { (2013) }\end{array}$ & $\begin{array}{l}36,774 \text { adults, } 18-70 \text { years- } \\
\text { old, } \\
\text { California Health Interview } \\
\text { Study } 2009\end{array}$ & $\begin{array}{l}3.2 \% \text { identified as LGB, and } \\
7.1 \% \text { of them were in same-sex } \\
\text { marriages, } 12.4 \text { in registered } \\
\text { domestic partnerships (RDP). }\end{array}$ & $\begin{array}{l}\text { Kesslers Psychological Distress } \\
\text { scale. }\end{array}$ & $\begin{array}{l}\text { Overall, ss effect (effect size n.a.). } \\
\text { Married HET had lowest levels, followed by LGB } \\
\text { same-sex married, HET unmarried, LGB in RDP, } \\
\text { and other LGB. No ss effect between LGB same- } \\
\text { sex Married, LGB in RDP and HET of any marital } \\
\text { status. } \\
\text { In paper, results are adjusted for self-rated health. } \\
\text { However, results remain the same if not adjusted } \\
\text { for self-related health (Wight, pers. } \\
\text { communication, April 2015) }\end{array}$ & & None reported & \\
\hline $\begin{array}{l}\text { Christchurch } \\
\text { Birth Cohort }\end{array}$ & $\begin{array}{l}\text { Complete birth cohort of } \\
\text { Christchurch, NZ, followed up } \\
\text { several times. }\end{array}$ & & & & & & \\
\hline $\begin{array}{l}\text { Fergusson, } \\
\text { Horwood, \& } \\
\text { Beautrais } \\
(1999)^{b}\end{array}$ & $\begin{array}{l}1,007 \text { young adults, } 21 \text { years- } \\
\text { old. }\end{array}$ & $\begin{array}{l}2.8 \% \text { SM individuals, } 2.0 \% \\
\text { identified as LGB and } 0.8 \% \text { as } \\
\text { heterosexuals but having } \\
\text { heterosexual relationships since } \\
\text { age } 16 \text {. } \\
\text { Interview }\end{array}$ & $\begin{array}{l}\text { Mental disorders 14-21 years; } \\
\text { past year mental disorders } \\
\text { (DSM-III-R) with Diagnostic } \\
\text { Interview for Children (at age } \\
\text { 15/16), mental disorders (DSM- } \\
\text { IV) with CIDl at ages } 18 \text { and } 21 . \\
\text { SA (lifetime) }\end{array}$ & $\begin{array}{l}\text { GAD: } 2.8, \text { ss } \\
\text { MD: } 4.0, \text { s } \\
\text { Conduct disorder: } 3.8, \text { ss } \\
\text { Nicotine dependence : } 5.0, \text { ss } \\
\text { Other substance abuse: } 1.9, \text { nss } \\
\text { Multiple disorders: } 5.9, \text { ss. } \\
\text { SA: } 6.2, \text { ss }\end{array}$ & None reported & None reported & \\
\hline $\begin{array}{l}\text { Fergusson, } \\
\text { Horwood, } \\
\text { Ridder, \& } \\
\text { Beautrais } \\
(2005)^{b}\end{array}$ & $\begin{array}{l}967 \text { young adults, } 25 \text { years } \\
\text { old. }\end{array}$ & $\begin{array}{l}+++ \\
12.4 \% \text { SM }(2.8 \% \text { moHO and } 9.6 \% \\
\text { moHET), based on a latent-class } \\
\text { variable created from } \\
\text { behavior/attraction/identity } \\
\text { dimensions at ages } 21 \text { and } 25 .\end{array}$ & $\begin{array}{l}\text { Mental disorders (DSM-IV) } 21- \\
25 \text { years; with CIDI at ages } 18, \\
21 \text { and } 25, \text { suicide attempts in } \\
\text { past } 5 \text { years with single item. }\end{array}$ & $\begin{array}{l}\text { Effects all ss if not specified as nss } \\
\text { Men: (moHO, moHET) } \\
\text { MD: } 14.1,4.4 \text {, Anx. Disorder: } 46.6,3.6 \\
\text { AD: n.a., } 2.2 \text { nss, DD: } 6.0,3.2, \text { SA: } 24.8 \text {, n.a. } \\
\text { Women (moHO, moHET) } \\
\text { MD: } 3.1,1.9 \text {, Anx. disorder: } 2.5 n s s, 1.9 n s s \\
\text { AD: n.a., } 2.0 \text { nss, DD: } 4.7,4.7, \text { SA: } 7.7,3.2 \text { nss }\end{array}$ & $\begin{array}{l}\text { Larger effects for moHO } \\
\text { compared to moHET } \\
\text { individuals }\end{array}$ & $\begin{array}{l}\text { Larger effects } \\
\text { among men, } \\
\text { especially moHO } \\
\text { men. }\end{array}$ & $\begin{array}{l}\text { Unusual } \\
\text { categorization, } \\
\text { resulting in } \\
\text { large group of } \\
\text { moHET }\end{array}$ \\
\hline \begin{tabular}{|l} 
Danish \\
Registry
\end{tabular} & $\begin{array}{l}\text { All adults of Denmark, linking } \\
\text { mortality data with the register } \\
\text { of marital status. }\end{array}$ & & & & & & \\
\hline $\begin{array}{l}\text { Qin, Agerbo, \& } \\
\text { Mortensen } \\
(2003)^{a, b}\end{array}$ & Mortality data 1981-1997 & Current marital status & Suicides & Ss effect, 4.3 & None reported & None reported & \\
\hline $\begin{array}{l}\text { Mathy, } \\
\text { Cochran, } \\
\text { Olsen, \& Mays } \\
(2011)^{b}\end{array}$ & Mortality data 1990-2001 & $\begin{array}{l}\text { Current or past registered } \\
\text { domestic same-sex partnership } \\
\text { (255 in year } 1990 \text { up to } 2348 \text { in } \\
\text { mid of } 2001 \text { among men and } 862- \\
3521 \text { among women, respectively) }\end{array}$ & Suicides & $\begin{array}{l}\text { Men: incidence RR } 8.2 \mathrm{ss} \\
\text { Women: incidence RR 1.7, nss. } \\
\text { Reference group: current or past (heterosexually) } \\
\text { married individuals }\end{array}$ & None reported & $\begin{array}{l}\text { Larger effect } \\
\text { among men }\end{array}$ & \\
\hline
\end{tabular}


Online supplemental material to Plöderl \& Tremblay (2015). Mental health of sexual minorities. A systematic review. International Review of Psychiatry. http://dx.doi.org/10.3109/09540261.2015.1083949

9

\begin{tabular}{|c|c|c|c|c|c|c|c|}
\hline $\begin{array}{l}\text { Frisch \& } \\
\text { Simonsen } \\
(2013)^{a, b}\end{array}$ & Mortality data 2000-2010 & $\begin{array}{l}\text { See Mathy et al. (2010) } \\
0.1 \% \text { of men and women were in a } \\
\text { same-sex registered partnership; } \\
1.9 \% \text { of women and } 1.0 \% \text { of men } \\
\text { were cohabiting in same } \\
\text { household at least one year. }\end{array}$ & Suicides & $\begin{array}{l}\text { For same-sex registered partners, ss incidence } \\
\text { Hazard Ratios (HR): Men: } 4.1 \text {, Women: } 6.4 \\
\text { Reference group: current or past heterosexually } \\
\text { married individuals. } \\
\text { For same-sex cohabiting individuals, HR } 3.2, \text { ss, } \\
\text { among men and HR } 1.8 \text { nss among women. }\end{array}$ & None reported & $\begin{array}{l}\text { Smaller effect } \\
\text { among men }\end{array}$ & \\
\hline $\begin{array}{l}\text { Dutch Survey } \\
\text { of General } \\
\text { Practice }\end{array}$ & $\begin{array}{l}\text { Adults, random sample from } \\
\text { lists of } 104 \text { general } \\
\text { practitioners }\end{array}$ & $\begin{array}{l}\text { Item on sexual preference (women } \\
\text { exclusively, predominantly women, } \\
\text { both women and men, men } \\
\text { predominantly, men only). } \\
\text { Predominantly and exclusively } \\
\text { categories were combined to HET } \\
\text { and HO. } \\
\text { Questionnaire }\end{array}$ & & & & & \\
\hline $\begin{array}{l}\text { T. G. M. } \\
\text { Sandfort, } \\
\text { Bakker, } \\
\text { Schellevis, \& } \\
\text { Vanwesenbeec } \\
\text { k (2006) }\end{array}$ & $\begin{array}{l}\text { 9,511 adults } \geq 18 \text { years-old } \\
\text { (mean 48.0-48.8 depending } \\
\text { on subgroup) }\end{array}$ & $\begin{array}{l}\text { 2.4\% identified as SM (1.5 HO, } 0.9 \\
\text { B) }\end{array}$ & $\begin{array}{l}\text { Mental health assessed with the } \\
\text { General Health Questionnaire } \\
\text { (GHQ), } \\
\text { current and ever smoking, soft } \\
\text { and hard drug use (ever), } \\
\text { binge drinking: } \geq 1 \text { day in past } 6 \\
\text { months. }\end{array}$ & 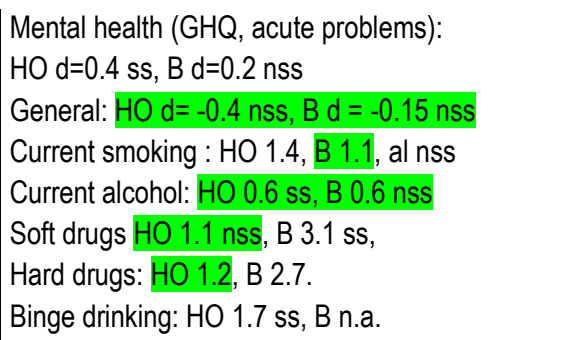 & $\begin{array}{l}\text { HO and B comparable for } \\
\text { smoking and alcohol. } \\
\text { Larger effect among B for } \\
\text { drug use. }\end{array}$ & $\begin{array}{l}\text { Larger effect for } \\
\text { women among B }\end{array}$ & \\
\hline \begin{tabular}{l|} 
T. G. M. \\
Sandfort, \\
Bakker, \\
Schellevis, \& \\
Vanwesenbeec \\
k (2009)
\end{tabular} & $\begin{array}{l}\text { 9,684 adults, } \geq 18 \text { years-old } \\
\text { (mean } 45.7-57.2 \text { depending } \\
\text { on subgroup) }\end{array}$ & $\begin{array}{l}+++ \\
2.4 \% \text { identified as SM (1.5 HO, } 0.9 \\
\text { B) }\end{array}$ & $\begin{array}{l}\text { Acute mental health problems } \\
\text { assessed with the GHQ. } \\
\text { Past year or lifetime episode of } \\
\text { severe depression or anxiety } \geq \\
2 \text { weeks. }\end{array}$ & 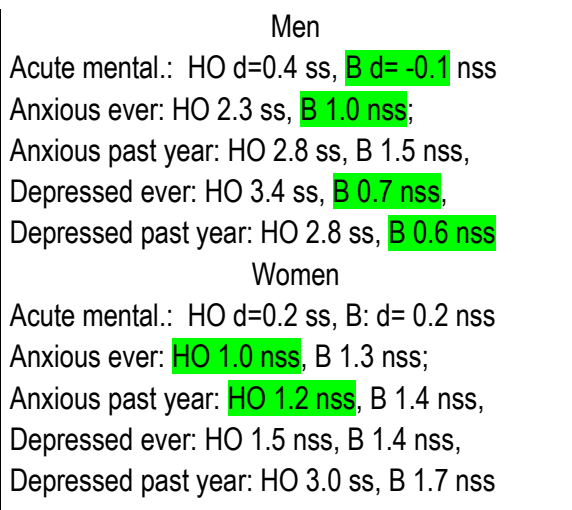 & $\begin{array}{l}\text { Among men, larger effects } \\
\text { for HO but not B (where } \\
\text { sometimes reversed } \\
\text { effects). } \\
\text { Comparable among } \\
\text { women. }\end{array}$ & $\begin{array}{l}\text { Larger effects for } \\
\text { HO men than } \\
\text { women, } \\
\text { smaller effect } \\
\text { among B men } \\
\text { than women. }\end{array}$ & \\
\hline $\begin{array}{l}\text { Los Angeles } \\
\text { County Health } \\
\text { Survey }\end{array}$ & $\begin{array}{l}\text { Adult women, USA, } \\
\text { Los Angeles. } \\
\text { Random Sample }\end{array}$ & $\begin{array}{l}\text { Sexual Identity. } \\
\text { Telephone Interview }\end{array}$ & & & & & \\
\hline $\begin{array}{l}\text { Diamant, Wold, } \\
\text { Spritzer, \& }\end{array}$ & $\begin{array}{l}4,697 \text { women, mean age } 42 \\
\text { years-old. }\end{array}$ & $\begin{array}{l}+++ \\
1.9 \% \text { LB }(0.8 \% \mathrm{~L})\end{array}$ & $\begin{array}{l}\text { Singe items on alcohol and } \\
\text { tobacco use }\end{array}$ & $\begin{array}{l}\text { All effect adjusted RRs (Table 2) } \\
\text { Current smoking: L } 1.7 \mathrm{ss}, \text { B } 1.7 \mathrm{ss}\end{array}$ & $L$ and $B$ comparable & - & $\begin{array}{l}\text { Low \% of SM } \\
\text { women, those }\end{array}$ \\
\hline
\end{tabular}


Online supplemental material to Plöderl \& Tremblay (2015). Mental health of sexual minorities. A systematic review. International Review of Psychiatry. http://dx.doi.org/10.3109/09540261.2015.1083949

\begin{tabular}{|c|c|c|c|c|c|c|c|}
\hline $\begin{array}{l}\text { Gelberg (2000) } \\
\text { b }\end{array}$ & Survey, 1997. & & & $\begin{array}{l}\text { Any alcohol: L } 1.1 \text { nss, B } 1.2 \mathrm{ss} \\
\text { Alcohol almost daily: L } 2.3 \mathrm{nss}, \text { B } 2.8 \mathrm{ss} \\
\geq 3 \text { drinks per sitting: L } 2.2 \mathrm{ss}, \text { B } 2.2 \mathrm{ss} \\
\geq 3 \text { drinks almost daily: L } 5.4 \mathrm{ss}, \text { B } 5.8 \mathrm{nss}\end{array}$ & & & $\begin{array}{l}\text { not sure of } \\
\text { their SO were } \\
\text { excluded }\end{array}$ \\
\hline $\begin{array}{l}\text { Diamant \& } \\
\text { Wold (2003) }\end{array}$ & $\begin{array}{l}\text { 4,135 women, mean age } 38 \\
\text { years old. } \\
\text { Survey } 1999 .\end{array}$ & $\begin{array}{l}+++ \\
2.7 \% \text { LB (1.7\% L) }\end{array}$ & $\begin{array}{l}\text { Single items on mental poor } \\
\text { mental health in past month ( } \geq 1 \\
\text { days), feeling depressed (all or } \\
\text { most of the time in past month), } \\
\text { receiving diagnosis of } \\
\text { depression or related disorder } \\
\text { by doctor, current smoking }\end{array}$ & $\begin{array}{l}\text { Unadjusted ORs (in paper adjustment for } \\
\text { smoking, obesity etc.) } \\
\text { Poor mental health: L L } 2.1 \text { ss, B } 1.3 \text { nss } \\
\text { Feeling depressed: L } 1.5 \text { nss, B } 1.7 \text { nss } \\
\text { Depression diagnosis: L } 2.3 \text { ss, B } 1.3 \text { nss } \\
\text { Smoking: L } 2.4 \text { ss, B } 2.7 \text { ss }\end{array}$ & $\begin{array}{l}\text { Smaller effects for B } \\
\text { compared to } L\end{array}$ & & \\
\hline $\begin{array}{l}\text { Midwestern } \\
\text { University, } \\
\text { under- } \\
\text { graduates }\end{array}$ & $\begin{array}{l}\text { Adults, USA, undergraduates } \\
\text { from a large Midwestern } \\
\text { university, 2003. } \\
\text { Random sample from } \\
\text { University register. }\end{array}$ & & & & & & \\
\hline $\begin{array}{l}\text { McCabe, } \\
\text { Hughes, } \\
\text { Bostwick, \& } \\
\text { Boyd }(2005)^{b}\end{array}$ & 9161 adults, age n.a. & $\begin{array}{l}+++ \\
12.1 \% \text { of women and } 9.1 \% \text { of men } \\
\text { identified as SM (moHET, B, } \\
\text { moLG, LG). } \\
17.6 \% \text { of women and } 8.9 \% \text { of men } \\
\text { were classified as SM on their } \\
\text { attraction (moHET, B, moHO, HO). } \\
3.7 \% \text { of men and } 3.9 \% \text { of women } \\
\text { were SM based on lifetime sexual } \\
\text { behavior (B, HO). } \\
\text { Web Survey }\end{array}$ & $\begin{array}{l}\text { Alcohol (binge drinking in past } \\
\text { two weeks), past year and past } \\
\text { month marijuana use, } \\
\text { other illicit drug use in past year }\end{array}$ & 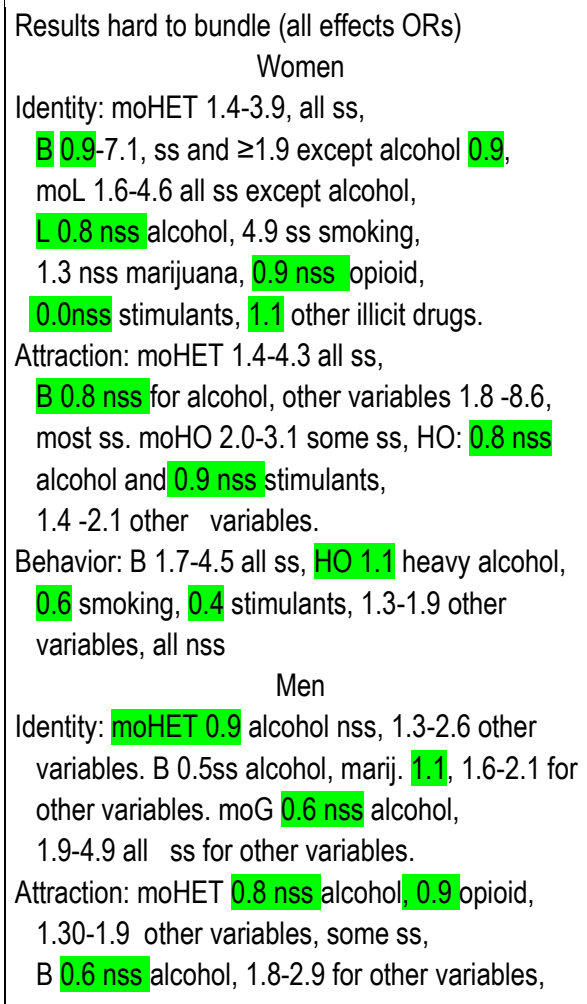 & $\begin{array}{l}\text { Complex pattern. } \\
\text { Generally all SM subgroups } \\
\text { more at risk, some ss, but } \\
\text { also some reversed effects } \\
\text { (including some ss). }\end{array}$ & $\begin{array}{l}\text { No clear } \\
\text { difference }\end{array}$ & \\
\hline
\end{tabular}




\begin{tabular}{|c|c|c|c|c|c|c|c|}
\hline & & & & $\begin{array}{l}\text { some ss. moHO } 0.5 \mathrm{ss} \text { alcohol,1.4-3.1 other } \\
\text { variables, some ss, } \mathrm{HO} 0.9 \text { alcohol, } 1.3-2.4 \text { for } \\
\text { other variables, some ss. } \\
\text { Behavior, B } 0.4 \mathrm{ss} \text { for alcohol, } 1.3-2.8 \text { for other } \\
\text { variables, some ss. HO } 0.6 \mathrm{ss} \text { alcohol, } 1.1 \mathrm{nss} \\
\text { opioids, } 1.3-1.8, \text { some ss, for other variables }\end{array}$ & & & \\
\hline $\begin{array}{l}\text { McCabe, } \\
\text { Hughes, } \\
\text { Bostwick, } \\
\text { Morales, \& } \\
\text { Boyd (2012) }\end{array}$ & $\begin{array}{l}2077 \text { adults, age n.a., who } \\
\text { completed both the 3- and } 5 \\
\text { category item on SO. }\end{array}$ & $\begin{array}{l}+++ \\
11.4 \% \text { identified as SM: LG } \\
(1.1 \%), \text { moLG }(0.9 \%), B(1.3 \%), \\
\text { moHET }(8.1 \%) . \\
\text { One forth of participants received } \\
\text { an additional item on identity (LG, } \\
\text { B, HET). Here, } 3.6 \% \text { identified as } \\
\text { LGB (1.7\% L, } 1.9 \% \text { B). } \\
\text { Web survey }\end{array}$ & $\begin{array}{l}\text { Past month cigarette use, binge } \\
\text { drinking ( } \geq 5 \text { drinks in a row, } \geq 4 \\
\text { for women) in past two weeks, } \\
\text { marijuana use in past year, } \\
\text { other illicit drug use in past year }\end{array}$ & 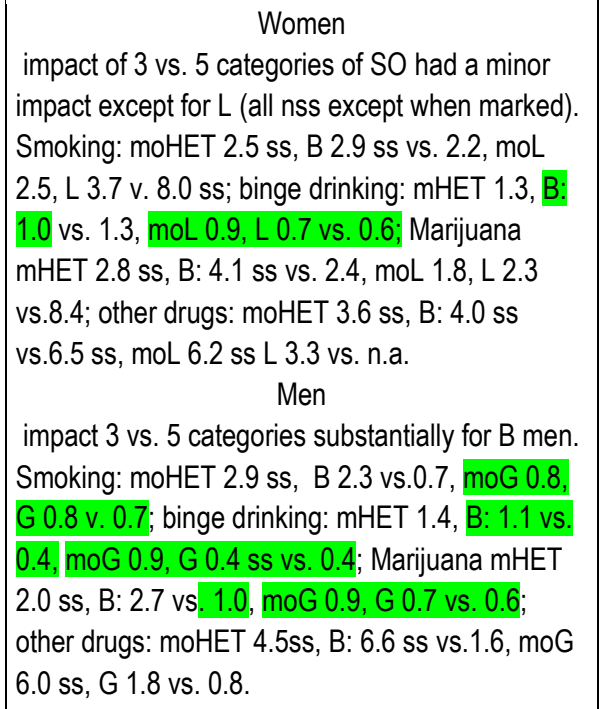 & $\begin{array}{l}\text { For women, comparable } \\
\text { effects, rather independent } \\
\text { of } 3 \text { vs. } 5 \text { categories } \\
\text { although among L increase } \\
\text { of effects for 5-categories. } \\
\text { For men, larger effects for } \\
\text { B than G in 3-category SO, } \\
\text { but B and G comparable in } \\
5 \text {-category SO and moHET } \\
\text { had largest effect. }\end{array}$ & \begin{tabular}{|l|} 
Women had \\
larger effects for \\
smoking, no clear \\
difference for \\
other outcomes
\end{tabular} & $\begin{array}{l}\text { Impact of } \\
\text { categories (3: } \\
\text { LGB vs. 5: } \\
\text { moHET/B/moL } \\
\text { G/LG) }\end{array}$ \\
\hline $\begin{array}{l}\text { National } \\
\text { Alcohol Surviey } \\
\text { (NAS) }\end{array}$ & $\begin{array}{l}\text { Adults, USA, } \\
\text { random sample }\end{array}$ & Telephone Interview & & & & & \\
\hline $\begin{array}{l}\text { Drabble, } \\
\text { Midanik, \& } \\
\text { Trocki (2005) }\end{array}$ & $\begin{array}{l}3,723 \text { adults, mean } 30.2-45.3 \\
\text { years-old (depending on } \\
\text { subgroup) } \\
1999-2001\end{array}$ & $\begin{array}{l}+++ \\
4.5 \% \text { SM in sample: } 1.2 \% \mathrm{HO} \\
\text { identified, } 1.1 \% \text { as B, } 2.0 \% \text { as } \\
\text { heterosexual with same-sex } \\
\text { sexual relationship in the past } 5 \\
\text { years (HetHo), and } 95.5 \% \mathrm{HET} \\
\text { identified with only HET } \\
\text { relationships in the past } 5 \text { years } \\
\text { telephone interview }\end{array}$ & $\begin{array}{l}\text { Items on drinking status, mean \# } \\
\text { drinks past year, mean \# of days } \\
\text { with } \geq 5 \text { drinks (heavy drinkers), } \\
\text { drunkenness } \geq 2 \text { times past, } \\
\text { drinking with social } \\
\text { consequences } \geq 2 \text { past year, } \\
\text { DSM-IV AD }\end{array}$ & $\begin{array}{l}\text { Women } \\
\text { All SM women less abstinent (0.4, nss). } \\
\text { Heavy drinking: L 5.2, B 5.8, HetHo } 3.3 \\
\text { Other variables: elevated for LB women. } \\
\text { AD: L } 5.3 \text { ss, B } 8.3 \text { ss, HetHo } 1.8 \text { nss } \\
\quad \text { Men } \\
\text { Male descriptives not given because of nss except } \\
\text { that G were less likely abstinent (0.3). } \\
\text { AD: G 2.1, B 1.2, HetHo 2.2, all nss }\end{array}$ & $\begin{array}{l}\text { Women: comparable levels } \\
\text { of alcohol use between } \\
\text { subgroups, for alcohol } \\
\text { dependency, B women } \\
\text { somewhat elevated } \\
\text { compared to L and HetHo. } \\
\text { Men: Larger effects for G } \\
\text { compared to G }\end{array}$ & $\begin{array}{l}\text { SO differences } \\
\text { larger among SM } \\
\text { women than SM } \\
\text { men. }\end{array}$ & \\
\hline $\begin{array}{l}\text { Ericksen \& } \\
\text { Trocki (1994) b }\end{array}$ & $\begin{array}{l}1,861 \text { adults, mean age } 44 \\
\text { years-old, } \\
\text { survey } 1990\end{array}$ & $\begin{array}{l}1.8 \% \text { of men and } 2.1 \% \text { of women } \\
\text { identified as } \mathrm{HO} \text { or } \mathrm{B} \text {, } \\
\text { telephone interview }\end{array}$ & $\begin{array}{l}\text { Problem drinking when } \geq 3 \\
\text { positive items in an 8-item index } \\
\text { about } A D \text { smptoms. }\end{array}$ & $\begin{array}{l}\text { Men: } 9.7 \text {, ss } \\
\text { Women: } 1.3 \text {, nss }\end{array}$ & None reported & $\begin{array}{l}\text { Substantially } \\
\text { larger effects for } \\
\text { men than women }\end{array}$ & \\
\hline Midanik, & 7,612 adults, $\geq 18$ years-old & +++ & Only DSM-IV AD reported here. & Women & For identity, $L G$ and $B$ are & For identity, larger & Term "inter- \\
\hline
\end{tabular}


Online supplemental material to Plöderl \& Tremblay (2015). Mental health of sexual minorities. A systematic review. International Review of Psychiatry. http://dx.doi.org/10.3109/09540261.2015.1083949

\begin{tabular}{|c|c|c|c|c|c|c|c|}
\hline $\begin{array}{l}\text { Drabble, } \\
\text { Trocki, \& Sell } \\
(2007)^{\mathrm{b}}\end{array}$ & $\begin{array}{l}\text { (mean age n.a.). } \\
\text { Surveys 1999-2001 }\end{array}$ & $\begin{array}{l}2.1 \% \text { of women and } 2.4 \% \text { of men } \\
\text { identified as LGB; } 3.1 \% \text { of women } \\
\text { and } 4.6 \% \text { of men had sexual } \\
\text { intercourse in the past } 5 \text { years }\end{array}$ & & \begin{tabular}{c} 
identity: L $5.5 \mathrm{ss}$, B 8.5 ss, \\
behavior: HO 1.2 nss, B 6.1 ss \\
\multicolumn{1}{c}{ Men (all nss) } \\
identity: G 1.9, B 0.9, \\
behavior: HO 1.8, B 2.3.
\end{tabular} & $\begin{array}{l}\text { comparable. } \\
\text { For behavior, B had larger } \\
\text { effects. }\end{array}$ & $\begin{array}{l}\text { effects among } \\
\text { women but } \\
\text { comparable for } \\
\text { behavior. }\end{array}$ & $\begin{array}{l}\text { course" } \\
\text { appropriate? }\end{array}$ \\
\hline $\begin{array}{l}\text { Netherlands } \\
\text { Mental Health } \\
\text { Survey and } \\
\text { Incidence } \\
\text { Study } \\
\text { (NEMESIS) }\end{array}$ & $\begin{array}{l}\text { Representative of population } \\
\text { in Netherlands. } \\
\text { Stratified random sample. }\end{array}$ & & $\begin{array}{l}\text { Composite International } \\
\text { Diagnostic Interview (CIDI). } \\
\text { Administered by trained } \\
\text { interviewers who are not } \\
\text { clinicians. }\end{array}$ & & & & \\
\hline $\begin{array}{l}\text { de Graaf, } \\
\text { Sandfort, \& ten } \\
\text { Have }(2006)^{\text {b }}\end{array}$ & $\begin{array}{l}7046 \text { adults (18-64 years), } \\
\text { survey } 1996\end{array}$ & $\begin{array}{l}2.8 \% \text { of men and } 1.4 \% \text { of women } \\
\text { had } \mathrm{HO} \text { contacts in the past year. } \\
7 \% \text { of those men and } 14 \% \text { of } \\
\text { those women were B active in the } \\
\text { past year, F2F interview }\end{array}$ & Lifetime SA from the CIDI & $\begin{array}{l}\text { Men 10.2, ss } \\
\text { Women: } 1.5, \text { nss }\end{array}$ & Not reported & $\begin{array}{l}\text { Large effect for } \\
\text { men but not } \\
\text { women. }\end{array}$ & \\
\hline $\begin{array}{l}\text { T. G. M. } \\
\text { Sandfort, de } \\
\text { Graaf, Bijl, \& } \\
\text { Schnabel } \\
(2001)^{b}\end{array}$ & $\begin{array}{l}\text { 5,998 adults, } 18-64 \text { years old } \\
\text { (mean ca. 40) }\end{array}$ & $\begin{array}{l}2.8 \% \text { of men and } 1.4 \% \text { of women } \\
\text { had } \mathrm{HO} \text { contacts in the past year. } \\
7 \% \text { of those men and } 14 \% \text { of } \\
\text { those women were B active in the } \\
\text { past year. } \\
\text { Telephone or } F 2 F(? ? ?) \text { interview. }\end{array}$ & & 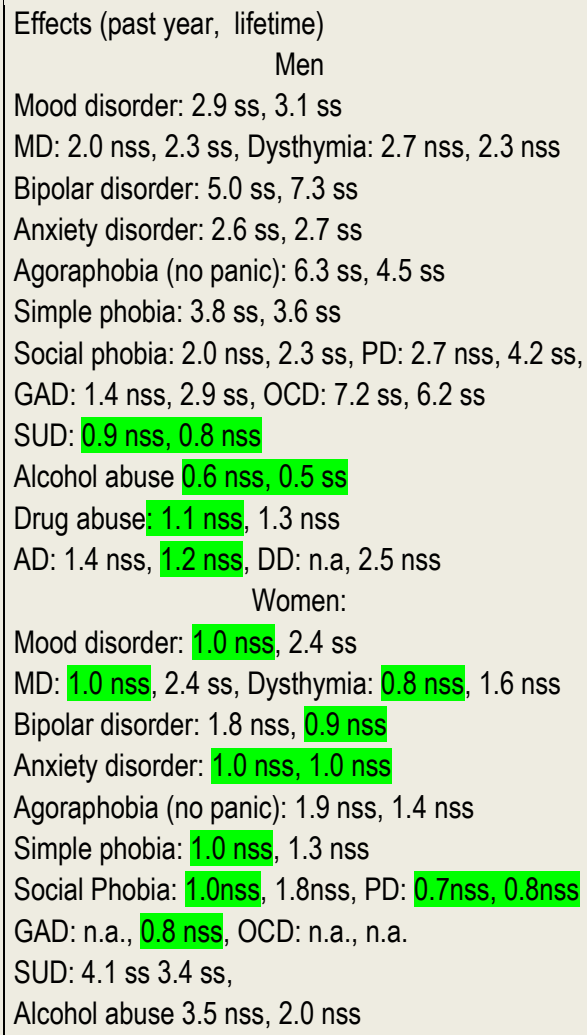 & None reported & $\begin{array}{l}\text { For mood and } \\
\text { anxiety disorders, } \\
\text { larger SO } \\
\text { differences } \\
\text { among men than } \\
\text { women, reversed } \\
\text { for substance use } \\
\text { disorders }\end{array}$ & \\
\hline
\end{tabular}




\begin{tabular}{|c|c|c|c|c|c|c|c|}
\hline & & & & $\begin{array}{l}\text { Drug abuse: n.a., } 1.9 \text { nss } \\
\text { AD: } 3.7 \text { nss, } 3.6 \text { ss, DD: } 4.4 \text { nss, } 8.0 \text { ss }\end{array}$ & & & \\
\hline $\begin{array}{l}\text { Gevonden et } \\
\text { al. (2014) }\end{array}$ & $\begin{array}{l}\text { 5,927 (NEMESIS 1) and 5,300 } \\
\text { (NEMESIS 2) adults, 18-64 } \\
\text { years-old ( mean ca. 40) }\end{array}$ & $\begin{array}{l}2.0 \% \text { (NEMESIS } 1 \text { ) and } 2.2 \% \\
\text { (NEMESIS 2) were classified as } \\
\text { SM based on HO contact in the } \\
\text { past year. NEMESIS } 2 \text { also } \\
\text { assessed sexual attraction and } 2 \% \\
\text { were then SM. }\end{array}$ & $\begin{array}{l}\text { Any psychotic symptom, CIDI } \\
\text { interview only or, if possible, } \\
\text { follow-up telephone interview } \\
\text { with SCID in case of positive } \\
\text { responses to psychotic } \\
\text { symptoms in CIDI. }\end{array}$ & $\begin{array}{l}\text { All effects ss } \\
\text { NEMESIS } 1: 3.3 \\
\text { NEMESIS 2: } \\
2.7 \text { for behavior } \\
2.3 \text { for attraction }\end{array}$ & None reported & None reported & \\
\hline $\begin{array}{l}\text { National Health } \\
\text { Interview } \\
\text { (NHIS) }\end{array}$ & $\begin{array}{l}\text { Representative sample of the } \\
\text { civilian, noninstitutionalized } \\
\text { adult population in the US. } \\
\text { Surveys } 1997 \text { and } 2012 \text {. }\end{array}$ & $\begin{array}{l}\text { All responses are self-reported in a } \\
\text { face-to-face interview }\end{array}$ & & & & & \\
\hline $\begin{array}{l}\text { Gonzales \& } \\
\text { Henning-Smith } \\
(2015)\end{array}$ & $\begin{array}{l}\text { 49,462 older cohabiting adults, } \\
\geq 50 \text { years-old, } \\
\text { surveys } 1997 \text { and } 2012 \text {. }\end{array}$ & $\begin{array}{l}0.5 \% \text { were living together with } \\
\text { someone of own sex in same } \\
\text { household who was described as } \\
\text { husband, wife, or partner, } \\
\text { F2F interview. }\end{array}$ & $\begin{array}{l}\text { Mental distress in past month } \\
\text { with Kesslers scale (cutoff } \geq 7 \\
\text { points, combined mild to severe } \\
\text { distress). }\end{array}$ & $\begin{array}{l}\text { Compared to HET married individuals, ss effects } \\
\text { among SM men (1.9) and women (1.6). However, } \\
\text { compared to unmarried partnered HETs, near } \\
\text { zero effect among men and reversed effect } \\
\text { among women. }\end{array}$ & None reported & $\begin{array}{l}\text { Smaller effect } \\
\text { among women }\end{array}$ & $\begin{array}{l}\text { Small number } \\
\text { of SO } \\
\text { individuals }\end{array}$ \\
\hline $\begin{array}{l}\text { Reczek, Liu, \& } \\
\text { Spiker (2014) }\end{array}$ & $\begin{array}{l}\text { 181,581 adults, } 18-65 \text { years- } \\
\text { old, } 1997-2011 \text { surveys. }\end{array}$ & $\begin{array}{l}\text { Same-sex married ( } 0.1 \%): 124 \\
\text { men }(15.2 \% \text { of male same-sex } \\
\text { couples), } 90 \text { women }(11.5 \%) \text { of } \\
\text { female same-sex couples). } \\
\text { Same-sex cohabiting }(0.8 \%): 693 \\
\text { men, } 692 \text { women. }\end{array}$ & $\begin{array}{l}\text { Alcohol use: lifetime abstainers, } \\
\text { current light drinking (0-3 drinks } \\
\text { per week), current moderate (4- } \\
14 \text { drinks weekly), current heavy } \\
\text { drinking (> } 14 \text { weekly drinks). } \\
\text { Psychological distress with the } \\
\text { Kessler scale }\end{array}$ & 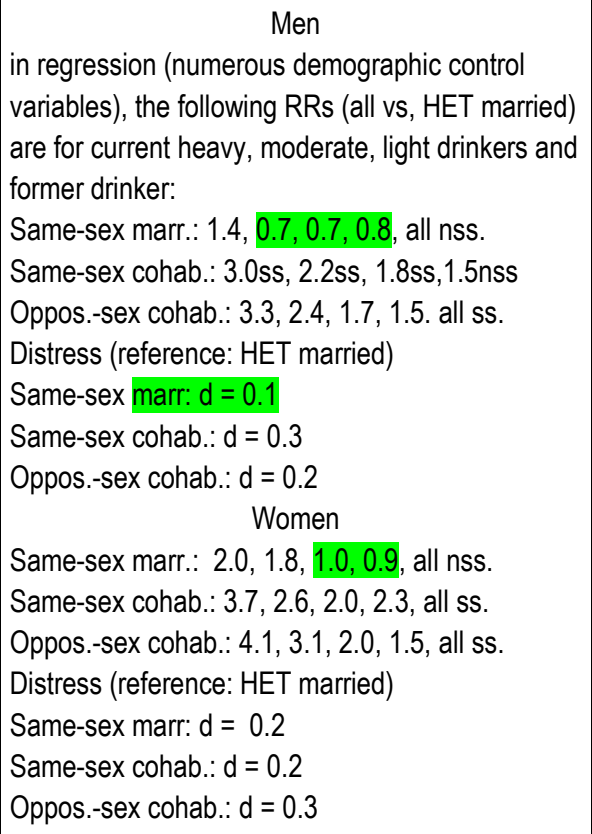 & $\begin{array}{l}\text { Similar results for same-sex } \\
\text { and opposite sex } \\
\text { cohabiting. Given that } \\
\text { same-sex married couples } \\
\text { form a small minority of all } \\
\text { same-sex couples (13.6\%), } \\
\text { and that opposite sex } \\
\text { married form the majority of } \\
\text { opposite sex couples ( } 81.6, \\
\text { Goodwin et al., 2010, 2002 } \\
\text { data), all same-sex couples } \\
\text { would more closely } \\
\text { approximate the RR } \\
\text { results of same-sex } \\
\text { cohabiting couples ( } 86.4 \% \\
\text { of SM couples) vs. HET } \\
\text { married couples ( } 81.6 \% \text { of } \\
\text { HET couples). } \\
\text { See Goodwin, Mosher, \& } \\
\text { Chandra (2010) }\end{array}$ & $\begin{array}{l}\text { Larger effects } \\
\text { among women. } \\
\text { Reversed effects } \\
\text { among men but } \\
\text { not among } \\
\text { women. }\end{array}$ & \\
\hline $\begin{array}{l}\text { National } \\
\text { Epidemiologic }\end{array}$ & $\begin{array}{l}\text { Adults } \geq 20 \text { years, USA, } \\
\text { random sample, complex }\end{array}$ & Home based F2F interviews & $\begin{array}{l}\text { AUDADIS-IV Interview to assess } \\
\text { DSM-IV disorders, in past year }\end{array}$ & & & & \\
\hline
\end{tabular}


Online supplemental material to Plöderl \& Tremblay (2015). Mental health of sexual minorities. A systematic review. International Review of Psychiatry. http://dx.doi.org/10.3109/09540261.2015.1083949

\begin{tabular}{|c|c|c|c|c|c|c|c|}
\hline $\begin{array}{l}\text { Survey on } \\
\text { Alcohol and } \\
\text { Related } \\
\text { Conditions } \\
\text { (NESARC) }\end{array}$ & $\begin{array}{l}\text { sampling design, } \\
\text { representative of the US } \\
\text { population }\end{array}$ & & and lifetime, SA with one item & & & & \\
\hline $\begin{array}{l}\text { Barnes, } \\
\text { Hatzenbuehler, } \\
\text { Hamilton, \& } \\
\text { Keyes (2014) }\end{array}$ & 34,653 adults, wave II & $1.7 \%$ identified as LGB & Past year and lifetime disorders & $\begin{array}{l}\text { All effects ss, (< bachelor, } \geq \text { bachelor ) } \\
\text { Past year disorders: } \\
\text { Any axis I disorder: } 3.5,1.8 \\
\text { any mood disorder: } 3.0,1.4 \\
\text { any anxiety disorder: } 2.9,1.6 \\
\text { any substance disorder: } 3.3,2.3 \\
\text { any comorbidity, } 3.9,2.3 \\
\text { Comparable results for lifetime }\end{array}$ & None reported & $\begin{array}{l}\text { Larger effects for } \\
\text { women for any } \\
\text { axis I disorder } \\
\text { and for any } \\
\text { substance } \\
\text { disorder } \\
\text { (in online } \\
\text { supplemental) }\end{array}$ & $\begin{array}{l}\text { Unadjusted } \\
\text { results used, } \\
\text { (adjusting for } \\
\text { marriage is } \\
\text { problematic) }\end{array}$ \\
\hline $\begin{array}{l}\text { Bolton \& } \\
\text { Sareen (2011) } \\
\text { b }\end{array}$ & 34,345 adults, Wave II & $\begin{array}{l}+++ \\
2 \% \text { identified as LGBQ }(0.9 \% \text { LG, } \\
0.6 \% \mathrm{~B}, 0.5 \% \mathrm{Q})\end{array}$ & $\begin{array}{l}\text { Lifetime DSM-IV disorders and } \\
\text { SA (any mood disorder, MD, } \\
\text { dysthymia, mania, hypomania, } \\
\text { any anxiety disorder, PD (with / } \\
\text { without agoraphobia), social } \\
\text { phobia, specific phobia, GAD }\end{array}$ & 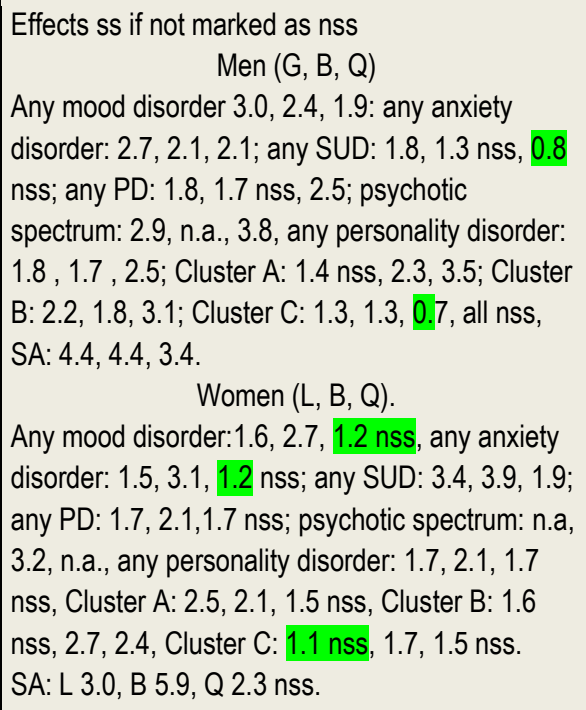 & $\begin{array}{l}\text { Among men, similar or } \\
\text { somewhat smaller SO } \\
\text { differences for B compared } \\
\text { to G men. Q men had } \\
\text { smaller SO differences } \\
\text { except for PD. } \\
\text { Among women, similar or } \\
\text { somewhat larger SO } \\
\text { differences for B than for L } \\
\text { women and generally } \\
\text { smaller SO differences for } \\
\text { Q women. }\end{array}$ & $\begin{array}{l}\text { Complex pattern, } \\
\text { for substance } \\
\text { abuse disorders } \\
\text { larger effects } \\
\text { among women, }\end{array}$ & $\begin{array}{l}\text { Adjusting for } \\
\text { marital status, } \\
\text { but changes in } \\
\text { results are } \\
\text { minor. }\end{array}$ \\
\hline $\begin{array}{l}\text { Bostwick, } \\
\text { Boyd, Hughes, } \\
\text { \& McCabe } \\
(2010)^{\text {b }}\end{array}$ & 34,653 adults, Wave II & $\begin{array}{l}+++ \\
5.8 \% \text { were nonheterosexual } \\
\text { attracted ( } 1.4 \% \text { only same-sex, } \\
0.4 \% \text { mostly same-sex, } 1.0 \% \\
\text { equal to both sexes, } 3 \% \text { mostly } \\
\text { opposite sex); } \\
4.4 \% \text { were nonheterosexual active } \\
\text { in lifetime ( } 1.5 \% \text { only same-sex, } \\
1.9 \% \text { B), } 1.9 \% \text { identified as LBGQ } \\
(0.8 \% \text { LG, } 0.6 \% \text { B, } 0.5 \% \text { Q) }\end{array}$ & $\begin{array}{l}\text { Any lifetime and any past year } \\
\text { DSM-IV mood and anxiety } \\
\text { disorders. }\end{array}$ & $\begin{array}{l}\text { Men: } \\
\text { Identity (G, B, Q) } \\
\text { Lifetime mood disorder: } 2.4,2.1,2.1 \text {, all ss } \\
\text { Lifetime MD: } 3.4,3.1,1.9 \text {, all ss } \\
\text { Lifetime anxiety disorder: } 2.7,2.7 .2 .2 \text {, all ss } \\
\text { Past year mood disorder: } 1.8 \mathrm{ss}, 1.7 \mathrm{nss}, 4.0 \mathrm{ss} \\
\text { Past year anx. disorder: } 2.3 \mathrm{ss}, 2.4 \mathrm{ss}, 2.0 \mathrm{nss} \\
\text { Attraction (HO, moHO, B, moHET) } \\
\text { Lifetime mood disorder: } 1.7,2.6,2.3,1.7 \text {, all ss } \\
\text { Lifetime MD: } 2.1,2.8,2.3,1.5 \text {, all ss. } \\
\text { Lifetime anx: } 2.2 \mathrm{ss}, 3.3 \mathrm{ss}, 1.6 \mathrm{nss}, 1.5 \mathrm{ss}\end{array}$ & $\begin{array}{l}\text { Men: behaviorally B men } \\
\text { but not B identified man } \\
\text { had somewhat larger SO } \\
\text { differences, and also } \\
\text { moHO attracted men. } \\
\text { Women: B women had the } \\
\text { largest SO differences } \\
\text { among all dimensions and } \\
\text { there were reversed SO } \\
\text { differences for HO attracted }\end{array}$ & $\begin{array}{l}\text { Smaller SO } \\
\text { differences } \\
\text { among women. } \\
\text { HO behaving and } \\
\text { attracted men had } \\
\text { higher risk than } \\
\text { heterosexuals but } \\
\text { the reversed was } \\
\text { true among } \\
\text { women (small } \\
\text { effects). }\end{array}$ & $\begin{array}{l}\text { Effects } \\
\text { adjusted for } \\
\text { marital status } \\
\text { and health } \\
\text { insurance } \\
\text { status - may } \\
\text { be biased } \\
\text { Results for } \\
\text { lifetime MD are } \\
\text { given }\end{array}$ \\
\hline
\end{tabular}




\begin{tabular}{|c|c|c|c|c|c|c|c|}
\hline & & & & 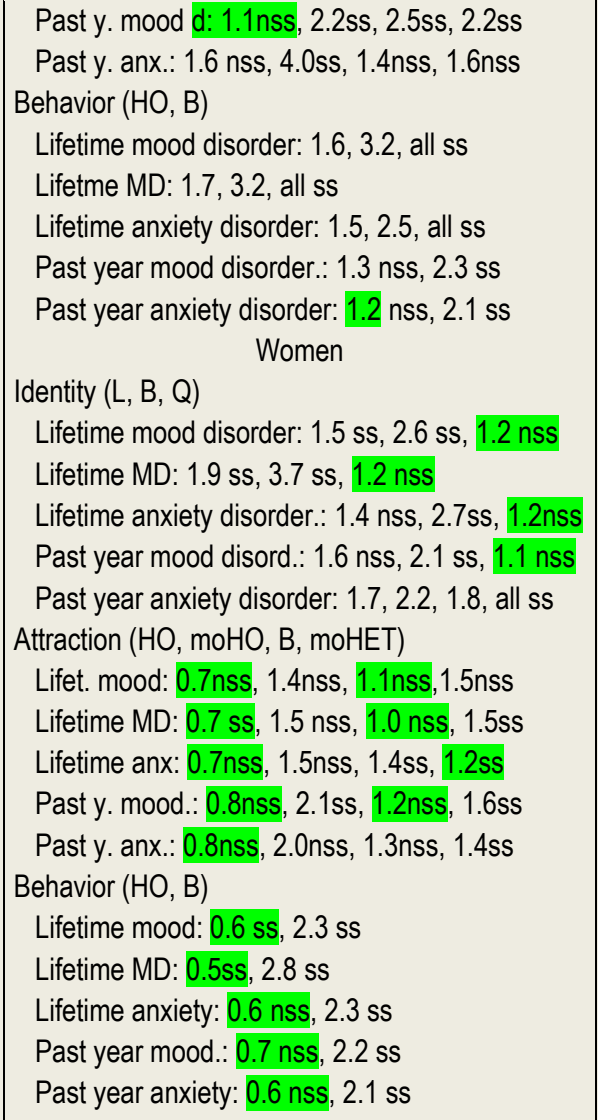 & and $\mathrm{HO}$ behaving women. & & unadjusted \\
\hline $\begin{array}{l}\text { Gattis, Sacco, } \\
\& \\
\text { Cunningham- } \\
\text { Williams (2012) }\end{array}$ & $\begin{array}{l}\text { 34,058/34,012 (depending on } \\
\text { definition of SO) adults, Wave } \\
\text { II }\end{array}$ & $\begin{array}{l}\text { 1.6\% were classified as } \\
\text { behaviorally concordant } \mathrm{HO} \\
\text { (becoHO), i.e., identified as } \mathrm{HO} \\
\text { and were ever } \mathrm{HO} \text { active, and } \\
2.0 \% \text { were behaviorally } \\
\text { heterosexual discordant } \\
\text { (bediscHET), i.e., identified as } \\
\mathrm{HET} \text { and were ever } \mathrm{HO} \text { active. } \\
\text { Similarly, } 1.4 \% \text { were concordant } \\
\mathrm{HO} \text { regarding sexual attraction } \\
\text { (attrcoHO) and } 1.6 \% \text { were } \\
\text { discordant for sexual attraction } \\
\text { and identity (attrdiscHET). }\end{array}$ & $\begin{array}{l}\text { Lifetime DSM-IV disorders } \\
\text { including MD, GAD, PTSD and } \\
\text { substance use disorders } \\
\text { (alcohol, narcotics, depressants, } \\
\text { stimulants, hallucinogen, } \\
\text { inhalants). }\end{array}$ & $\begin{array}{l}\text { HET concordant and HO concordant were used } \\
\text { as baseline. } \\
\text { Behavioral Discordance: } \\
\text { Men: bediscHET: SUD 1.3-3.0 (except AD } 0.8 \\
\text { ss and depressants } 1.1 \text { nss), MD } 1.9 \text { nss, GAD } \\
1.3 \text { nss, PTSD } 2.1 \text { ss. compared to HET. } \\
\text { BediscHET had had lower risk than beconHO } \\
\text { (0.3-0.9, ss for AD 0.5 and GAD 0.4). } \\
\text { Women: bediscHET SUD 3.3-8.3: MD, GAD and } \\
\text { PTSD (1.4-2.4, all ss). BediscHET had smaller } \\
\text { risk than beconHO (0.7-1.0, all nss except MD) for } \\
\text { all disorders except inhalants (2.5, nss) and PTSD } \\
\text { (1.1, nss). } \\
\text { Attraction discordance: } \\
\text { Men: attrdiscHET SUD 0.2-0.4, all nss except }\end{array}$ & $\begin{array}{l}\text { HET identified with HO } \\
\text { experience generally were } \\
\text { between concordant HETs } \\
\text { (lowest risk) and } \\
\text { concordant } \mathrm{HO} \text { (highest } \\
\text { risk). } \\
\text { In contrast, HET identified } \\
\text { with } \mathrm{HO} \text { attraction were at } \\
\text { generally at lower risk than } \\
\text { concordant HET and much } \\
\text { lower than concordant HO, } \\
\text { except among men for MD, } \\
\text { GAD, and PTSD } \\
\text { (comparable levels with }\end{array}$ & $\begin{array}{l}\text { Concerning } \\
\text { behavioral } \\
\text { discordance, } \\
\text { discordant men } \\
\text { were closer to } \\
\text { HETS and } \\
\text { discordant women } \\
\text { were closer to } \\
\text { concordant HO. } \\
\text { More complex } \\
\text { pattern for } \\
\text { attraction } \\
\text { discordance }\end{array}$ & $\begin{array}{l}\text { Multiple } \\
\text { measure of SO }\end{array}$ \\
\hline
\end{tabular}


Online supplemental material to Plöderl \& Tremblay (2015). Mental health of sexual minorities. A systematic review. International Review of Psychiatry. http://dx.doi.org/10.3109/09540261.2015.1083949

\begin{tabular}{|c|c|c|c|c|c|c|c|}
\hline & & & & $\begin{array}{l}\text { AD; MD 1.4, GAD 1.3, PTSD 1.1 (all nss), } \\
\text { attrdiscHET had lower risk compared with } \\
\text { attrconHO (0.1-0.5), most ss. } \\
\text { Women: attrdiscHET SUD }(0.7-0.9 \text {, all nss), MD } \\
\text { (0.4, nss), GAD, PTSD (all 0.6, ss) and they were } \\
\text { at lower risk than attrconHO }(0.2-0.3 \text {, nearly all ss) }\end{array}$ & concordant HETS). & & \\
\hline $\begin{array}{l}\text { Hatzenbuehler, } \\
\text { Keyes, \& Hasin } \\
\text { (2009), b same } \\
\text { as Barnes, et } \\
\text { al. (2014) }\end{array}$ & $\begin{array}{l}34,653 \text { adults, } \\
\text { wave II }\end{array}$ & Similar to Barnes et al (2014) & Similar to Barnes et al (2014) & $\begin{array}{l}\text { All effects ss except for Dysthymia } 1.4 \text {. } \\
\text { Any disorder: } 2.4 \text {, more than to disorders: } 3.7 \text {, } \\
\text { any mood disorder: } 2.3, \text { MD: } 2.5 \text {, mania: } 2.0 \text {, } \\
\text { any anxiety disorder: } 2.3, \text { GAD: } 2.4 \text {, } \\
\text { social anxiety: } 2.9 \text {, specific phobia: } 1.9, \text { PTSD: } \\
2.2, \text { PD: } 3.5 \text {, any SUD: } 2.6, \text { AD: } 2.9, \text { DD: } 5.7 \text {. }\end{array}$ & None reported & None reported & $\begin{array}{l}\text { ORs slightly } \\
\text { larger than in } \\
\text { paper } \\
\text { (adjusted for } \\
\text { marriage } \\
\text { status) }\end{array}$ \\
\hline $\begin{array}{l}\text { Hughes, } \\
\text { McCabe, } \\
\text { Wilsnack, } \\
\text { West, \& Boyd } \\
\text { (2010) }\end{array}$ & $\begin{array}{l}34,653 \text { adults, } \\
\text { wave II }\end{array}$ & $\begin{array}{l}+++ \\
2 \% \text { identified as LGBQ }(0.9 \% \text { LG, } \\
0.6 \% \mathrm{~B}, 0.5 \% \mathrm{Q})\end{array}$ & $\begin{array}{l}\text { Any SUD in past year (AD and } \\
\text { DD dependency/abuse) }\end{array}$ & $\begin{array}{l}\text { Effects all RRs: } \\
\text { Women: L } 4.4 \text { ss, B } 4.2 \text { ss, Q 2.2, nss. } \\
\text { Men: G } 2.0 \text { ss, B } 1.8 \text { nss, Q } 1.1 \text { nss }\end{array}$ & $\begin{array}{l}\text { LG comparable with } B \text {, } \\
\text { smaller effects for } Q\end{array}$ & $\begin{array}{l}\text { Larger SO } \\
\text { difference for } \\
\text { women than men }\end{array}$ & \\
\hline $\begin{array}{l}\text { McCabe, } \\
\text { Hughes, } \\
\text { Bostwick, } \\
\text { West, \& Boyd } \\
(2009)^{b}\end{array}$ & $\begin{array}{l}34653 \text { adults, } \\
\text { Wave II }\end{array}$ & $\begin{array}{l}+++ \\
2 \% \text { identified as LGBQ, } 4 \% \text { had a } \\
\text { least one lifetime same-sex sexual } \\
\text { partner, and } 6 \% \text { were sexually } \\
\text { attracted to some degree to the } \\
\text { same sex. }\end{array}$ & $\begin{array}{l}\text { Past year AD, marijuana } \\
\text { dependency, other DD (past } \\
\text { year heavy drinking, marijuana } \\
\text { use and other drug use not } \\
\text { reported for simplicity). }\end{array}$ & 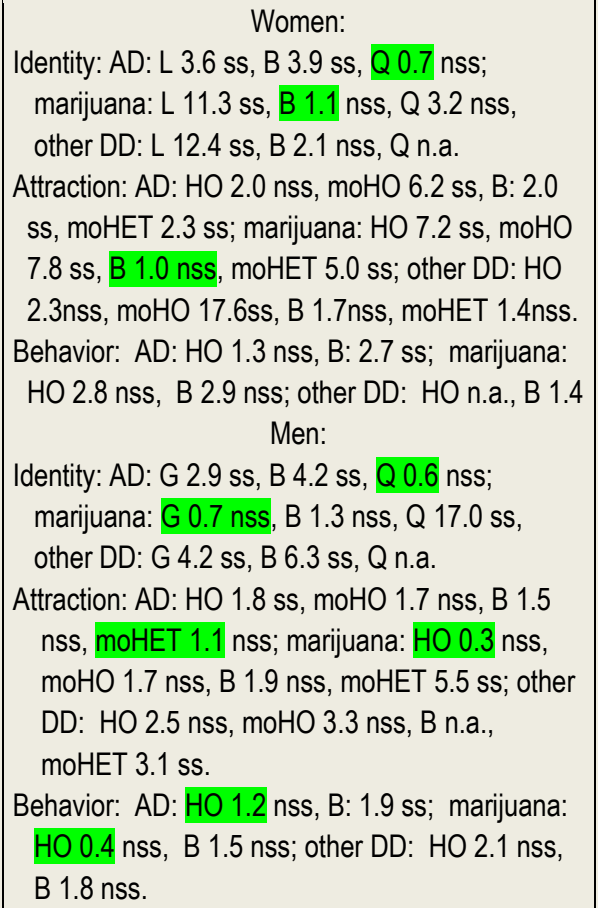 & $\begin{array}{l}\text { Among women, B generally } \\
\text { smaller effects than } L \text { or } \\
\text { moL (except behavior) and } \\
\text { not sure somewhat smaller } \\
\text { effects. } \\
\text { Large effects among } \\
\text { moHET attracted and L } \\
\text { identified. } \\
\text { Among men, B identified } \\
\text { somewhat larger effects } \\
\text { than } G \text { but comparable for } \\
\text { attraction and behavior. } \\
\text { No clear pattern for } Q \\
\text { identified or other } \\
\text { subgroups. }\end{array}$ & $\begin{array}{l}\text { Somewhat larger } \\
\text { effects among } \\
\text { women but not for } \\
\text { all subgroups. }\end{array}$ & \\
\hline National Health & Adults, cluster-stratified, & At home visits and in mobile & & & & & \\
\hline
\end{tabular}


Online supplemental material to Plöderl \& Tremblay (2015). Mental health of sexual minorities. A systematic review. International Review of Psychiatry. http://dx.doi.org/10.3109/09540261.2015.1083949

\begin{tabular}{|c|c|c|c|c|c|c|c|}
\hline $\begin{array}{l}\text { and Nutrition } \\
\text { Examination } \\
\text { Survey } \\
\text { (NHANES) }\end{array}$ & $\begin{array}{l}\text { multistage probability sample, } \\
\text { USA }\end{array}$ & $\begin{array}{l}\text { trailers, Questionnaires, Interviews } \\
\text { (mental disorders), sensitive } \\
\text { information with ACASI and CAPI } \\
\text { (since 1992/1993) }\end{array}$ & & & & & \\
\hline $\operatorname{CDC}(2013) b$ & $\begin{array}{l}\text { 7,011 men ( } 20-59 \text { years-old). } \\
\text { Surveys } 1999-2008\end{array}$ & $\begin{array}{l}5.1 \% \text { of men were classified as } \\
\text { SM based on } \mathrm{HO} \text { contacts, } \\
\text { lifetime. }\end{array}$ & $\begin{array}{l}\text { Having ever used a needle to } \\
\text { take street drugs or having used } \\
\text { a needle to inject drugs not } \\
\text { prescribed by a doctor. }\end{array}$ & $\begin{array}{l}\text { SM men were overrepresented among injecting } \\
\text { drug users compared to nonusers (10.5 vs. } 4.7 \% \text {, } \\
\text { RR 2.2, ss?). }\end{array}$ & None reported & - & \\
\hline $\begin{array}{l}\text { Cochran \& } \\
\text { Mays }(2000 \mathrm{a})^{\mathrm{b}} \\
\text { "Lifetime } \\
\text { prevalence..." }\end{array}$ & $\begin{array}{l}3,648 \text { men (17-39 years-old), } \\
\text { Surveys 1988-1994 }\end{array}$ & $\begin{array}{l}2.2 \% \text { of men reported "sexual } \\
\text { intercourse" with other men, } 87 \% \\
\text { of them also reported intercourse } \\
\text { with women. }\end{array}$ & $\begin{array}{l}\text { Lifetime Affective DSM-III } \\
\text { Disorders and SA with } \\
\text { Diagnostic Interview Schedule, }\end{array}$ & $\begin{array}{l}\text { MD } 2.4 \text { nss, single episode MD } 1.9 \text { nss, } \\
\text { recurrent MD } 3.6 \text { nss, Bipolar I } 0.6 \text { nss, } \\
\text { Atypical bipolar } 2.3 \text { nss, Dysthymia } 1.2 \text { nss } \\
\text { SA: } 5.4 \text { ss }\end{array}$ & None reported & - & $\begin{array}{l}\text { Low \% SM, } \\
\text { "sexual } \\
\text { intercourse" } \\
\text { problematic }\end{array}$ \\
\hline $\begin{array}{l}\text { Cochran \& } \\
\text { Mays (2011) }\end{array}$ & $\begin{array}{l}5,574 \text { men (17-59 years-old), } \\
\text { survey } 1988-1994, \text { matched } \\
\text { with National Death Index in } \\
18 \text { year follow up period }\end{array}$ & $\begin{array}{l}1 \% \text { were classified as SM based } \\
\text { on any same-sex sexual contact }\end{array}$ & Death by suicide & $\begin{array}{l}\text { No SM men died by suicide compared to } 0.3 \% \text { of } \\
\text { heterosexual men, very weak evidence, } \\
\text { Bayesian OR } 2.3 \text { (Credible Interval } 0.1 \text { - 13.4) } \\
\text { (see Plöderl et al., } 2013 \text { for calculation details). }\end{array}$ & None reported & - & $\begin{array}{l}\text { Low \% SM, } \\
\text { "sexual } \\
\text { intercourse" } \\
\text { problematic }\end{array}$ \\
\hline $\begin{array}{l}\text { Farmer, } \\
\text { Bucholz, Flick, } \\
\text { Burroughs, \& } \\
\text { Bowen (2013) }\end{array}$ & $\begin{array}{l}7,078 \text { men ( } \geq 20 \text { years-old), } \\
\text { USA, } \\
\text { Surveys 2001-2010 }\end{array}$ & $\begin{array}{l}5.2 \% \text { identified as LGB or had at } \\
\text { least one same-sex sexual partner } \\
\text { in life. Q and "something else" } \\
\text { were excluded }\end{array}$ & $\begin{array}{l}\text { Current smoking status, illicit } \\
\text { drug use excluding marijuana } \\
\text { (ever, lifetime), current risky } \\
\text { drinker based on NIAAA criteria }\end{array}$ & $\begin{array}{l}\text { Near similar rate of smoking status, } \\
\text { drug use (1.8 ss), } \\
\text { reversed effect for risky drinking }(0.9, \mathrm{nss}) \text {. }\end{array}$ & None reported & - & $\begin{array}{l}\text { Mixture of SO } \\
\text { dimensions. }\end{array}$ \\
\hline $\begin{array}{l}\text { Farmer, } \\
\text { Jabson, } \\
\text { Bucholz, \& } \\
\text { Bowen }(2013)^{b}\end{array}$ & $\begin{array}{l}5,793 \text { adult women }(\geq 20 \\
\text { years-old), USA, Surveys } \\
2001-2008\end{array}$ & $\begin{array}{l}8.2 \% \text { identified as LGB (or } \\
\text { "something else") or had at least } \\
\text { one same-sex sexual partner in } \\
\text { life, } Q \text { were excluded }\end{array}$ & $\begin{array}{l}\text { Current smoking status, illicit } \\
\text { drug use excluding marijuana } \\
\text { (ever, lifetime), current risky } \\
\text { drinker based on NIAAA criteria }\end{array}$ & $\begin{array}{l}\text { All effects ss } \\
\text { Smoking (2.1), drug use (2.2), and risky drinking } \\
(2.8) \text {. }\end{array}$ & None reported & $\begin{array}{l}\text { Comparable } \\
\text { (Farmer et al. } \\
\text { 2013a) for drug } \\
\text { use, larger effects } \\
\text { for women for } \\
\text { smoking/ drinking }\end{array}$ & $\begin{array}{l}\text { Mixture of SO } \\
\text { dimension, } \\
\text { "something } \\
\text { else"-defined } \\
\text { included }\end{array}$ \\
\hline $\begin{array}{l}\text { Przedworski, } \\
\text { McAlpine, } \\
\text { Karaca- } \\
\text { Mandic, \& } \\
\text { VanKim (2014) } \\
\text { b }\end{array}$ & $\begin{array}{l}\text { Women subsample, women }(\geq \\
20 \text { years-old) } \\
2001-2010\end{array}$ & $\begin{array}{l}+++ \\
9.3 \% \text { were classified as } L \text { women } \\
\text { who have sex with women (WSM), } \\
\text { B WSW, and HET WSW }\end{array}$ & $\begin{array}{l}\text { Alcohol, average } \geq 7 \text { drinks per } \\
\text { week in past year), } \geq 1 \text { day binge } \\
\text { drinking in past year, smoking (> } \\
100 \text { cigarettes, lifetime). }\end{array}$ & $\begin{array}{l}\text { Effects are all unadjusted RRs not including } \\
\text { relationship status, provided by the author (pers. } \\
\text { comm.) All effecst ss if not marked as nss. } \\
\text { Heavy alcohol use: L 2.2, B 2.0, HET WSW } 1.8 \\
\text { Binge drinking: L 1.4 nss, B 1.5, HET WSW } 1.6 \\
\text { Smoking: L 1.5, B 1.7, HET WSW } 1.8\end{array}$ & $\begin{array}{l}\text { Comparable effects among } \\
\text { all subgroups. }\end{array}$ & - & \\
\hline $\begin{array}{l}\text { Nurses Health } \\
\text { Study }\end{array}$ & $\begin{array}{l}\text { Registered female nurses, } \\
\text { USA }\end{array}$ & & & & & & $\begin{array}{l}\text { Very few SM } \\
\text { women in } \\
\text { sample }\end{array}$ \\
\hline $\begin{array}{l}\text { Case et al. } \\
(2004)^{b}\end{array}$ & $\begin{array}{l}\text { 90,823 women ( } 28-43 \text { years- } \\
\text { old), survey } 1995\end{array}$ & $\begin{array}{l}1.2 \% \mathrm{SM} \text { women, } 0.8 \% \text { of the } \\
\text { women identified as } \mathrm{L}, 0.3 \% \mathrm{~B} \text {, }\end{array}$ & $\begin{array}{l}\text { Medical Outcome Study Short } \\
\text { Form (SF-36 mental index } \leq\end{array}$ & $\begin{array}{l}\text { All effects RR (Prevalence Ratios) } \\
\text { Current smoking: L 2.0, B 2.2, } 0 \text { 1.3, all ss }\end{array}$ & $\begin{array}{l}\text { No difference for smoking,. } \\
\text { Comparable effects for }\end{array}$ & - & \\
\hline
\end{tabular}


Online supplemental material to Plöderl \& Tremblay (2015). Mental health of sexual minorities. A systematic review. International Review of Psychiatry. http://dx.doi.org/10.3109/09540261.2015.1083949

\begin{tabular}{|l|l|l|}
\hline & & $\begin{array}{l}\text { and 0.1\% that none applied, } \\
\text { postal questionnaire }\end{array}$ \\
\hline $\begin{array}{l}\text { Jun et al. } \\
\text { (2010) }\end{array}$ & $\begin{array}{l}\text { Around 62,200 (\# differs by } \\
\text { outcome variable), 25-42 } \\
\text { years-old. } \\
\text { Survey 2001 }\end{array}$ & $\begin{array}{l}+++ \\
\text { Identified as L (0.8\%) or B }(0.3) \\
\text { ("other" respondents and those } \\
\text { who preferred not to answer were } \\
\text { excluded) }\end{array}$ \\
\hline
\end{tabular}

52), ever using antidepressants. Smoking status and number of cigarettes per day

Alcohol: estimated grams daily, Heavy drinking defined as $\geq 60$ drinks per month

Early Smoking (by age 14) and between 15 and 19

Early drinking (by ages 15-17)
Current $\geq 15$ cig.daily: $L$ 1.1ss, B 1.0nss

L 1.9 ss, B 2.7 ss, 00.5 nss

Heavy drinkers: L 1.7 ss, B 3.2ss, 0 0.7nss

SF MH Index: $L$ 1.4, B 1.6, 0 1.3, all ss

Antidepressants: L 1.8, B 1.7, 0 1.4, all ss

Early smoking (RRs $L$ 2.2, B 3.2). Smoking age 15-19 and early drinking (both RRs L: 1.5, B 1.6).

\# Cigarettes daily (15-19 years):

$L d=0.2 \mathrm{ss}, B d=0.3 \mathrm{ss}$

Grams daily alcohol ( $15-19$ years):

$L d=0.3 \mathrm{ss}, B d=0.1 \mathrm{nss}$
Alcohol use ( $>15 \mathrm{~g} /$ day):

amounts of alcohol.

$B$ more likely heavy

drinkers.

Comparable for depression

and use of antidepressants

No ss difference between $\mathrm{L}$.

Early alcohol and $B$

smoking

\section{SEPARATE STUDIES}

\begin{tabular}{|c|c|c|c|c|c|c|c|}
\hline $\begin{array}{l}\text { Bagley \& } \\
\text { Tremblay } \\
\text { (1997), (1998) }\end{array}$ & $\begin{array}{l}750 \text { adult men, age } 18-27 \\
\text { years-old, Canady, Calgary, } \\
1992 . \\
\text { Stratified random sample. }\end{array}$ & \begin{tabular}{|l|}
+++ \\
$10.9 \%$ identified as homo- or \\
bisexual or had same sex contacts \\
in the past 6 months. \\
Computer questionnaire in private \\
space.
\end{tabular} & $\begin{array}{l}\text { Depression (CES-D Scale, Cut- } \\
\text { Off } \geq 28 \text { ), items on lifetime SA } \\
\text { (with intent to die). }\end{array}$ & $\begin{array}{l}\text { SA: } 14.4, \text { ss } \\
\text { Depression ( } \geq 28 \text { ): } \\
\text { Sexual active: } \mathrm{HO}: 2.5 \mathrm{nss}, \mathrm{B}: 4.6 \mathrm{ss} \\
\text { Celibate: } \mathrm{HO} 3.1 \mathrm{nss} \\
\text { Depression (dimensional): } \mathrm{ss} \text {, effect size } \\
\text { Sexually active: } \mathrm{HO} d=0.1, \mathrm{~B} d=0.2 \\
\text { Celibate: } \mathrm{HO}: \mathrm{d}=0.2\end{array}$ & $\begin{array}{l}\text { B slightly more SA and } \\
\text { depression. }\end{array}$ & - & $\begin{array}{l}\text { Stronger } \\
\text { definition of SA }\end{array}$ \\
\hline $\begin{array}{l}\text { Berg et al. } \\
(2014)\end{array}$ & $\begin{array}{l}24,055 \text { College students, } \\
\text { USA, mean age } 23.5 \text { years- } \\
\text { old, from } 6 \text { southeast colleges, } \\
\text { random sample }\end{array}$ & $\begin{array}{l}2.9 \% \text { identified as } \mathrm{HO}, 3.7 \% \text { as } \mathrm{B} \text {, } \\
\text { web survey }\end{array}$ & $\begin{array}{l}\text { Alcohol and drug use similar to } \\
\text { the YRBS, alcohol or drug use } \\
\text { before sexual intercourse in this } \\
\text { study }\end{array}$ & $\begin{array}{l}\text { Alcohol/drugs prior to last sexual intercourse: } \\
\text { HO } 1.7 \text { and B 2.0, all ss }\end{array}$ & $\begin{array}{l}\text { Comparable SO- } \\
\text { differences for B and HO } \\
\text { participants }\end{array}$ & None reported & $\begin{array}{l}\text { Low response } \\
\text { rate }(21 \%)\end{array}$ \\
\hline $\begin{array}{l}\text { Bloomfield } \\
\text { (1993) }\end{array}$ & $\begin{array}{l}445 \text { adult women, } 18-50 \\
\text { years-old, USA, San } \\
\text { Francisco, 1987, random } \\
\text { sample }\end{array}$ & $\begin{array}{l}15 \% \text { identified as } \mathrm{B}(1 \%), \mathrm{moHO} \text {, } \\
\text { or } \mathrm{HO}, 85 \% \text { as moHET or } \mathrm{HET} \text {, } \\
\text { Mailed questionnaires }\end{array}$ & $\begin{array}{l}\text { Single items (Cahalan et al., } \\
\text { 1996) on amount of alcohol in } \\
\text { past month, perceived drinking } \\
\text { status (non-drinker, problem } \\
\text { drinker etc.), frequency bar- } \\
\text { going, alcohol before sexual } \\
\text { encounter }\end{array}$ & $\begin{array}{l}\text { SM more problematic levels: } \\
\text { Recovering alcoholics } 4.8 \mathrm{ss} \\
\text { Frequent bar goers, BF = } 18.29 \text {. } \\
\text { Perceived problem drinker: } 26.1 \mathrm{ss} \\
\text { Heavy drinking } 2.1 \mathrm{nss} \\
\text { Evidence for zero effects: BF } 4.8 \text { for \# of drinks, } \\
\text { BF } 4.1 \text {, mean drinks per day BF } 5.7 \text {, and drinking } \\
\text { before sexual encounter, BF } 3.2\end{array}$ & Not reported & - & \\
\hline $\begin{array}{l}\text { Bloomfield, } \\
\text { Wicki, } \\
\text { Wilsnack, } \\
\text { Hughes, \& } \\
\text { Gmel (2011) }\end{array}$ & $\begin{array}{l}34,270 \text { adults (mostly }>18 \\
\text { years-old), } 14 \text { different } \\
\text { countries } 2000-2007 \text {, } \\
\text { GENACIS study. For every } \\
\text { SM individual, } 15 \text { HET were } \\
\text { matched. Different sampling }\end{array}$ & $\begin{array}{l}0.7 \% \text { categorized as SM according } \\
\text { to having romantic same-sex } \\
\text { partners recently or in the past } \\
\text { year. } \\
\text { Questionnaire }\end{array}$ & $\begin{array}{l}\text { High volume drinking according } \\
\text { to WHO and heavy/binge } \\
\text { drinking by using amount of } \\
\text { alcohol for certain time periods }\end{array}$ & $\begin{array}{l}\text { For high volume drinking, and for all countries } \\
\text { together, ss effect for SM women (2.2) but not } \\
\text { men (1.0 nss). Ss effect for women only in North } \\
\text { America (3.6) and New Zealand but not in Europe. } \\
\text { For men, only ss effect in New Zealand. } \\
\text { For binge drinking, SO women had higher odds }\end{array}$ & None reported & $\begin{array}{l}\text { SO difference } \\
\text { rather among } \\
\text { women but } \\
\text { not/less/reversed } \\
\text { among men }\end{array}$ & $\begin{array}{l}\text { Few individuals } \\
\text { classified as } \\
\text { SM }\end{array}$ \\
\hline
\end{tabular}


Online supplemental material to Plöderl \& Tremblay (2015). Mental health of sexual minorities. A systematic review. International Review of Psychiatry. http://dx.doi.org/10.3109/09540261.2015.1083949

\begin{tabular}{|c|c|c|c|c|c|c|c|}
\hline & $\begin{array}{l}\text { (regional, national, probability/ } \\
\text { replacement) and modes of } \\
\text { administration). }\end{array}$ & & & $\begin{array}{l}\text { overall }(2.5, \mathrm{ss}) \text { and in most regions }(1.9-23.6) \\
\text { whereas partly reversed effect for men } 0.9, \text { nss } \\
(0.9-1.7 \text { in different regions) }\end{array}$ & & & \\
\hline $\begin{array}{l}\text { Booth et al. } \\
(2012)\end{array}$ & $\begin{array}{l}\text { 1,004 female veterans, USA, } \\
20-52 \text { years-old, } \\
\text { Midwestern Veterans Affairs } \\
\text { register. }\end{array}$ & $\begin{array}{l}11.1 \% \text { had partnership with } \\
\text { females or with both males and } \\
\text { females before, during, or after } \\
\text { military service; computer assisted } \\
\text { telephone interview }\end{array}$ & $\begin{array}{l}\text { Current MD according DSM-IV } \\
\text { with CIDI-SF, current PTSD with } \\
\text { the self-report PTSD scale, } \\
\text { lifetime SUD with the Substance } \\
\text { Abuse Outcomes Module }\end{array}$ & $\begin{array}{l}\text { SM status correlated significantly with depression } \\
(r=.12), \text { PTSD }(r=0.10) \text {, current SUD }(r=0.06) \text {. }\end{array}$ & None reported & - & \\
\hline $\begin{array}{l}\text { Bowring, Vella, } \\
\text { Degenhardt, } \\
\text { Hellard, \& Lim } \\
\text { (2015) }\end{array}$ & $\begin{array}{l}3793 \text { (66\% female), 16-29 } \\
\text { years-old (median 18.9), } \\
\text { 2011-2013, music festival } \\
\text { Melbourne Australia; }\end{array}$ & $\begin{array}{l}9 \% \text { of males and } 11 \% \text { of females } \\
\text { were identifying as } \mathrm{GLBQ} \text { or } \\
\text { Queer, } \\
\text { questionnaire }\end{array}$ & $\begin{array}{l}\text { Drinking alcohol per week, } \\
\text { smoking, illicit drug use, ever } \\
\text { injecting drugs, substance use, } \\
\text { sexual risk behavior, mental } \\
\text { health problems }\end{array}$ & $\begin{array}{l}\text { All effects ss. } \\
\text { Drinking } \geq 6 \text { drinks in a row weekly } 1.6, \\
\text { Smoking } 2.1 \text {, recent drug use } 1.7 \\
\text { ever injected drugs } 5.7 \\
\text { mental health problems in the past six months } 2.7 ; \\
\text { rated mental health as fair or poor } 3.0\end{array}$ & None reported & None reported & $\begin{array}{l}\text { Not sure if } \\
\text { prespecified } \\
\text { sample but } \\
\text { rates of SM } \\
\text { comparable to } \\
\text { population. }\end{array}$ \\
\hline $\begin{array}{l}\text { Browne, Clubb, } \\
\text { Wang, \& } \\
\text { Wagner (2009) }\end{array}$ & $\begin{array}{l}1837 \text { African American male } \\
\text { freshmen (17-19 years-old); } \\
2001 \text { HBCU Survey; south } \\
\text { and mid Atlantic US }\end{array}$ & $\begin{array}{l}6 \% \text { of men had same-sex sexual } \\
\text { contacts, } \\
\text { Self-administered survey } \\
\text { questionnaire. }\end{array}$ & $\begin{array}{l}\text { Substance use before recent } \\
\text { sexual encounter }\end{array}$ & Ss effect 1.8 & None reported & - & \\
\hline $\begin{array}{l}\text { Burgard, } \\
\text { Cochran, \& } \\
\text { Mays }(2005)\end{array}$ & $\begin{array}{l}11,204 \text { adult women (> } 18 \\
\text { years-old), California Womens } \\
\text { Health Survey, three waves } \\
\text { combined: } 1998,1999,2000 . \\
\text { Random digit dialing } \\
\text { telephone sample. }\end{array}$ & $\begin{array}{l}3.8 \% \text { of the women had at least } \\
\text { one HO partner in the past } 5 \text { years } \\
\text { (wave 1998), or lifetime (waves } \\
1999,2000 \text { ). } \\
\text { Among the HO experienced } \\
\text { women, in the } 1999-2000 \\
\text { subsample, } 33.5 \% \text { reported only } \\
\text { female sexual partners in the } \\
\text { past year, } 16.6 \% \text { both male and } \\
\text { female sexual partners, } 11.6 \% \\
\text { no sexual partners, and } 38.3 \% \\
\text { only male sexual partners. } \\
\text { Assessed by telephone interview. }\end{array}$ & $\begin{array}{l}\text { Single items for any current } \\
\text { smoking status and on alcohol } \\
\text { use in past month, binary } \\
\text { variables created: any alcohol, } \\
\text { once or more a week, } 5-7 \text { days } \\
\text { per week, engaged in binge } \\
\text { drinking, engaged in heavy } \\
\text { drinking ( } \geq 5 \text { binge drinking). } \\
\text { Ordinal variables: Number } \\
\text { drinking days, Number of drinks } \\
\text { per drinking day, Number of } \\
\text { binge drinking day. }\end{array}$ & $\begin{array}{l}\text { Smoking status: } 1.8, \mathrm{ss}, \\
\text { \#drinking days: } d=0.2 \mathrm{ss} \\
\text { \#drinks per drinking day: } d=0.3 \mathrm{ss} \\
\text { \#binge drinking days: } d=0.3 \mathrm{ss} \\
\text { Alcohol at least weekly } 1.8 \mathrm{ss}, \\
\text { Average } 5-7 \text { days drinking / week: } 1.1 \text { nss } \\
\text { Binge drinking: } 2.2 \mathrm{ss} \\
\text { Heavy drinking: } 2.7 \mathrm{ss} \\
\text { For alcohol, the effects were larger among 26-35 } \\
\text { year olds }\end{array}$ & $\begin{array}{l}\text { No difference for smoking } \\
\text { For alcohol, the SO } \\
\text { differences were larger } \\
\text { among B active women }\end{array}$ & - & \\
\hline $\begin{array}{l}\text { Burgess, Tran, } \\
\text { Lee, \& van Ryn } \\
(2007)\end{array}$ & $\begin{array}{l}7884 \text { adults, mean age ca. } 40 \\
\text { years-old, SHAPE survey, } \\
2003, \text { Minneapolis Minnesota. } \\
\text { Stratified random sample of } \\
\text { households, and one person } \\
\text { randomly from households. }\end{array}$ & $\begin{array}{l}3.7 \% \text { of women and } 9.1 \% \text { of men } \\
\text { identified as LGBT. } \\
\text { Telephone interviews. }\end{array}$ & $\begin{array}{l}\text { Mental health diagnoses by } \\
\text { doctor (ever), past month days } \\
\text { with psychological distress, } \\
\text { \# smoking and binge drinking in } \\
\text { past month. }\end{array}$ & $\begin{array}{l}\text { Ss effects for smoking (RR 1.5), } \\
\text { Past month binge drinking (OR } 1.6 \mathrm{ss} \text { ), } \\
\text { mean \# cigarettes, mean \# days with poor mental } \\
\text { health, diagnoses of depression (RR } 2.1 \mathrm{ss} \text { ), } \\
\text { anxiety disorders (RR } 2.1 \mathrm{ss} \text { ) }\end{array}$ & None reported & None reported & \\
\hline Chakraborty, & 7461 adults, & $8.7 \%$ were SM based on & Neurotic symptoms, common & All effects ss if not marked as nss. & Comparable effects for the & None reported & \\
\hline
\end{tabular}


Online supplemental material to Plöderl \& Tremblay (2015). Mental health of sexual minorities. A systematic review. International Review of Psychiatry. http://dx.doi.org/10.3109/09540261.2015.1083949

\begin{tabular}{|c|c|c|c|c|c|c|c|}
\hline $\begin{array}{l}\text { McManus, } \\
\text { Brugha, } \\
\text { Bebbington, \& } \\
\text { King (2011) }\end{array}$ & $\begin{array}{l}\text { representative sample of the } \\
\text { population living in private UK } \\
\text { households (APMS, 2007) }\end{array}$ & $\begin{array}{l}\text { identification (LG, B, moHET) and } \\
8.9 \% \text { based on having had any } \\
\text { same gender sexual partners. } \\
\text { Interviewer-administered } \\
\text { structured interview and computer } \\
\text { assisted questionnaire. }\end{array}$ & $\begin{array}{l}\text { mental disorders (CIS-R) in the } \\
\text { week prior to the interview, } \\
\text { probable psychosis (SCAN), } \\
\text { lifetime SA, past year AD and } \\
\text { DD. }\end{array}$ & $\begin{array}{l}\text { Identity: neurotic disorders } 1.5, \mathrm{MD} 1.8, \mathrm{GAD} 1.5 \text {, } \\
\mathrm{OCD} 2.2 \text {; phobic disorder } 1.9 \text {, probable psychosis } \\
3.8, \mathrm{SA} 2.2, \mathrm{AD} 2.1 \text {, and } \mathrm{DD} 1.7 \text {. } \\
\text { Behavior: neurotic disorders } 1.5, \mathrm{MD} 1.9, \mathrm{GAD} \\
1.5, \mathrm{OCD} 2.8 \text {; phobic disorder } 1.9, \text { probable } \\
\text { psychosis } 3.1, \mathrm{SA} 1.9, \mathrm{AD} 1.3 \mathrm{nss}, \mathrm{DD} 1.4 \mathrm{nss} \text {. }\end{array}$ & $\begin{array}{l}\text { identity and behavior } \\
\text { measures of } S O \text { except for } \\
A D \text { and } D D \text { (smaller effects } \\
\text { for behavioral measures) }\end{array}$ & & \\
\hline $\begin{array}{l}\text { Cochran, } \\
\text { Mays, Alegria, } \\
\text { Ortega, \& } \\
\text { Takeuchi } \\
\text { (2007) }\end{array}$ & $\begin{array}{l}4649 \text { Latino and Asian, } \geq 18 \\
\text { years-old, National Latino and } \\
\text { Asian American Survey } \\
\text { (NLAAS) }\end{array}$ & $\begin{array}{l}4.8 \% \text { were classified as SM based } \\
\text { on LGB identity or past year } \\
\text { sexual behavior. } \\
\text { F2F interview. }\end{array}$ & $\begin{array}{l}\text { Past year and lifetime } \\
\text { psychiatric morbidity (depressive } \\
\text { disorders, anxiety disorders, } \\
\text { substance use disorder, eating } \\
\text { disorder); } \\
\text { SA using modules from the } \\
\text { WMH-CIDI }\end{array}$ & 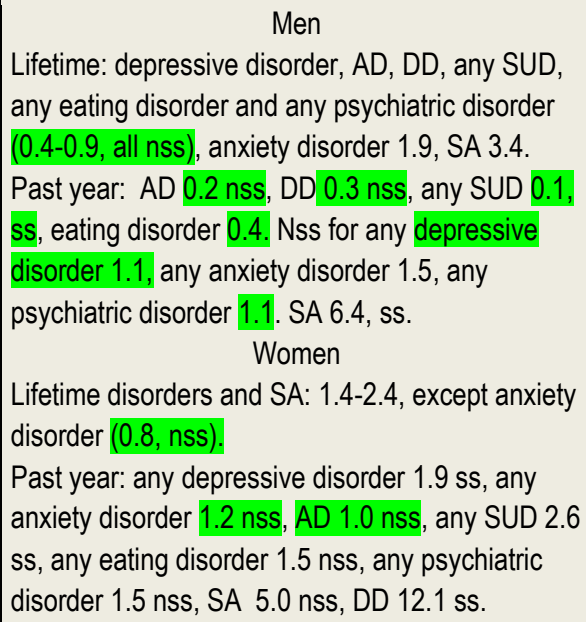 & None reported & $\begin{array}{l}\text { Larger effects } \\
\text { among women, } \\
\text { several reversed } \\
\text { effects among } \\
\text { men. }\end{array}$ & \\
\hline $\begin{array}{l}\text { Cochran \& } \\
\text { Mays (2000b) }\end{array}$ & $\begin{array}{l}\text { 9,908 adults, USA, random } \\
\text { sample, National Household } \\
\text { Survey of Drug Abuse } \\
\text { (NHSDA), } 1996\end{array}$ & $\begin{array}{l}2.4 \% \text { of men and } 1.6 \% \text { of women } \\
\text { had sex with same-sex persons in } \\
\text { the past year, about one third } \\
\text { among these had sex with men } \\
\text { and women } \\
\text { Interview }\end{array}$ & $\begin{array}{l}\text { Interview-Scales with cutoff- } \\
\text { values with high } \\
\text { correspondence to DSM-III/IV } \\
\text { classification, disorders for the } \\
\text { past } 12 \text { months, detailed } \\
\text { assessment of AD according to } \\
\text { Cochran et al. }(2000)\end{array}$ & $\begin{array}{l}\text { All mental health disorders (MD, GAD, } \\
\text { agoraphobia, panic attack, } A D, D D \text {, any disorder): } \\
\text { 1.3-4.9. } \\
\text { Ss effects for MD (men only), Panic attacks (men } \\
\text { only), DD (women only), } A D \text { (women only), any } \\
\text { psychiatric syndrome (men only). }\end{array}$ & $\begin{array}{l}\text { Reported as not being } \\
\text { different, but related } \\
\text { statistics not given. }\end{array}$ & $\begin{array}{l}\text { Larger effects } \\
\text { among men } \\
\text { except AD/DD } \\
\text { where men had } \\
\text { smaller effects. }\end{array}$ & $\begin{array}{l}\text { Nearly all SM } \\
\text { suicide } \\
\text { attempters in } \\
\text { age group 17- } \\
29 .\end{array}$ \\
\hline $\begin{array}{l}\text { Cochran, } \\
\text { Sullivan, \& } \\
\text { Mays (2003) }\end{array}$ & $\begin{array}{l}\text { 2,917 adults (25-74 years-old), } \\
\text { random sample, USA, MIDUS- } \\
\text { Survey }\end{array}$ & $\begin{array}{l}2.9 \% \text { of men and } 2.3 \% \text { of women } \\
\text { identified as } \mathrm{HO} \text { or } \mathrm{B}(1.1 \% \text { as } \mathrm{B}) \text {, } \\
\text { questionnaire }\end{array}$ & $\begin{array}{l}\text { DSM-III-R disorders in past year } \\
\text { with CIDI (Composite } \\
\text { International Interview), items for } \\
\text { AD/DD according to DSM-IV, } \\
\text { distress indicators with single } \\
\text { items, diagnosis of } \\
\text { anxiety/depression/other } \\
\text { emotional disorder (combined) } \\
\text { or alcohol/drug problem) }\end{array}$ & 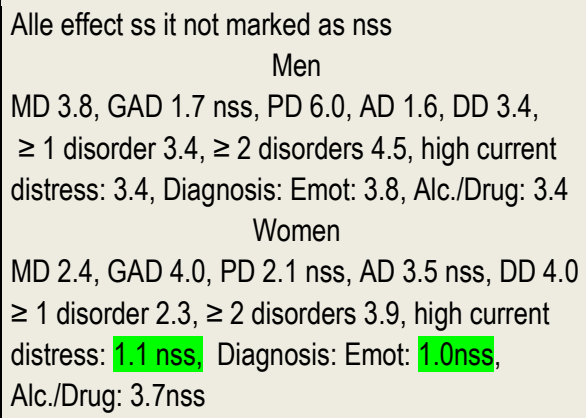 & None reported & $\begin{array}{l}\text { SO-Differences } \\
\text { for MD, PD, one } \\
\text { or more disorders: } \\
m>w \\
\text { For } G A D, A D \\
D D: w>m\end{array}$ & $\begin{array}{l}\text { Unadjusted } \\
\text { ORs calculated } \\
\text { because in } \\
\text { paper } \\
\text { adjustment for } \\
\text { relationship } \\
\text { status. }\end{array}$ \\
\hline $\begin{array}{l}\text { Cochran \& } \\
\text { Mays }(2015)\end{array}$ & $\begin{array}{l}17,886 \text { adults, } \geq 18 \text { years-old, } \\
1988-2002 \text { General Social }\end{array}$ & $\begin{array}{l}4.8 \% \text { were classified as SM based } \\
\text { on any lifetime number of same- }\end{array}$ & Death by suicide & $\begin{array}{l}\text { SM men had lower rates of suicides ( } 0.2 \text { vs. } 0.6 \% \text {, } \\
\text { RR } 0.3 \text {, adjusted HR } 0.4 \text {, nss). }\end{array}$ & None reported & $\begin{array}{l}\text { Large SO } \\
\text { difference among }\end{array}$ & \\
\hline
\end{tabular}


Online supplemental material to Plöderl \& Tremblay (2015). Mental health of sexual minorities. A systematic review. International Review of Psychiatry. http://dx.doi.org/10.3109/09540261.2015.1083949

\begin{tabular}{|c|c|c|c|c|c|c|c|}
\hline & $\begin{array}{l}\text { Surveys (representative } \\
\text { multistage probability sample) } \\
\text { linked with the National Death } \\
\text { Index with social security } \\
\text { numbers }\end{array}$ & $\begin{array}{l}\text { sex partner or in the year before } \\
\text { the interview. } \\
\text { Self administered questionnaire or } \\
\text { computer assisted self } \\
\text {-interview (CASI). }\end{array}$ & & $\begin{array}{l}\text { SM women had higher rates of suicides ( } 0.9 \text { vs. } \\
0.1 \%, \text { RR } 9.0 \text {, adjusted HR } 6.3 \mathrm{ss} \text { ). } \\
\text { Unadjusted RRs were calculated, because HRs in } \\
\text { paper were adjusted for mental health which } \\
\text { would biases the results. }\end{array}$ & & $\begin{array}{l}\text { women, reversed } \\
\text { effect among men }\end{array}$ & \\
\hline $\begin{array}{l}\text { Cheng, } \\
\text { Gipson, Perez, } \\
\text { \& Cochran } \\
(2014)\end{array}$ & $\begin{array}{l}\text { 1,112 young adults, } \\
20-22 \text { years-old, Philippines, } \\
\text { Cebu Longitudinal Health and } \\
\text { Nutrition Survey (CLHNS), all } \\
\text { index children of pregnant } \\
\text { women in } 33 \text { communities } \\
1982 / 83\end{array}$ & $\begin{array}{l}15.1 \% \text { of sexually experienced } \\
\text { were classified as SM based on } \\
\text { lifetime HS behavior or same-sex } \\
\text { romantic relationship. } \\
\text { Assessment methods: described } \\
\text { as interview, but CES-D scale is } \\
\text { questionnaire. }\end{array}$ & $\begin{array}{l}\text { CES-D Scale, } \\
\text { ever consuming nicotine, } \\
\text { alcohol, drugs. }\end{array}$ & $\begin{array}{l}\text { CES-D: women d=0.2, men } \mathrm{d}=0.1 \text {, all nss } \\
\text { Smoking: women } 3.6 \mathrm{ss} \text {, men } 2.8 \mathrm{ss} \\
\text { Alcohol: women } 2.3 \mathrm{nss} \text {, men n.a. } \\
\text { Drugs: women } 6.2 \mathrm{ss} \text {, men } 1.8 \mathrm{ss}\end{array}$ & None reported & $\begin{array}{l}\text { For substance } \\
\text { abuse, larger } \\
\text { effects for women }\end{array}$ & \\
\hline $\begin{array}{l}\text { M. Eisenberg \& } \\
\text { Wechsler } \\
(2003)\end{array}$ & $\begin{array}{l}10,301 \text { young adults, mostly } \\
18-22 \text { years-old, randomly } \\
\text { selected from undergraduate } \\
\text { students, USA, } 1999 \text { CAS } \\
\text { (College Alcohol Study) }\end{array}$ & $\begin{array}{l}+++ \\
6 \% \text { SM, } 2 \% \text { of women and } 3 \% \text { of } \\
\text { men only had } \mathrm{HO} \text { behavior; } \\
\text { percentages for both same-and } \\
\text { opposite sexual behavior was } 5 \\
\text { and } 2 \% \text {, respectively. } \\
\text { Self-administered questionnaires } \\
\text { mailed to students. }\end{array}$ & $\begin{array}{l}\text { Tobacco, alcohol and marijuana } \\
\text { use with single items, binge } \\
\text { drinking as } 4 \text { or } 5 \text { drinks in a } \\
\text { row. (Note: see Ford \& Jasinski, } \\
2005 \text {, for additional analyses) }\end{array}$ & $\begin{array}{l}\text { Smoking: B women } 2.1 \text {, ss, all other SM groups } \\
\text { 1.1-1.2, all nss. } \\
\text { Binge drinking: B women } 1.4 \mathrm{ss}, \mathrm{HO} \text { women } 1.0 \\
\text { nss, B men } 0.5 \mathrm{ss}, \mathrm{HO} \text { men } 0.6 \text {, nss. } \\
\text { Marijuana use: B women } 2.8 \mathrm{ss}, \mathrm{B} \text { men } 1.3 \mathrm{nss} \text {, } \\
\text { HO men and women } 0.8, \mathrm{nss} \text {. }\end{array}$ & $\begin{array}{l}\text { Slightly larger SO } \\
\text { differences for B men and } \\
\text { women except for binge } \\
\text { drinking among men (no } \\
\text { difference) and marijuana } \\
\text { use among women (effects } \\
\text { clearly larger for B than HO } \\
\text { women. }\end{array}$ & $\begin{array}{l}\text { SM men have } \\
\text { lower rates of } \\
\text { binge drinking } \\
\text { than HET men. }\end{array}$ & \\
\hline $\begin{array}{l}\text { Frisell, } \\
\text { Lichtenstein, } \\
\text { Rahman, \& } \\
\text { Langstrom } \\
\text { (2010) }\end{array}$ & $\begin{array}{l}17,379 \text { adult twins (20-47 } \\
\text { years-old), Sweden, } \\
\text { national twin registry/STAGE } \\
\text { study, all living twins born } \\
\text { 1959-1985, survey 2005/2006 }\end{array}$ & $\begin{array}{l}\text { SM based on any same-sex } \\
\text { sexual partners in life. } \\
\text { Mostly web-survey but also } \\
\text { interview and written questionnaire }\end{array}$ & $\begin{array}{l}\text { Lifetime MD, GAD, AD with } \\
\text { SCID according to DSM-IV, } \\
\text { ADHD according to DSM-IV, } \\
\text { current depression with CES-D }\end{array}$ & $\begin{array}{l}\text { All effects ss (men, women) } \\
\text { MD 1.8, 2.0, GAD 3.3, 1.9, } \\
\text { AD 1.6, 3.0, ADHD 1.4, 1.5. } \\
\text { CES-D scale, ss effects: } \\
\text { men } d=0.3 \text {, women } d=0.3\end{array}$ & None reported & $\begin{array}{l}\text { Comparable for } \\
M D \text { and } A D H D \text {, } \\
\text { larger effect for } \\
\text { women for } A D \\
\text { lower for } G A D\end{array}$ & \\
\hline $\begin{array}{l}\text { Ford \& Jasinski } \\
(2006)\end{array}$ & $\begin{array}{l}\text { 9,389 students, USA, age n.a. } \\
\text { but typically } \geq 18 \text { years-old, } \\
\text { Harvard School of Public } \\
\text { Health College Alcohol Study, } \\
\text { 1999, nested random } \\
\text { sampling, representative of } \\
\text { US colleges/universities. }\end{array}$ & $\begin{array}{l}+++ \\
2.4 \% \mathrm{HS} \text { and } 3.7 \% \mathrm{~B} \text { based on } \\
\text { lifetime sexual behavior (those } \\
\text { sexually inexperienced excluded). } \\
\text { Self-administered questionnaire. }\end{array}$ & $\begin{array}{l}\text { Past month marijuana and other } \\
\text { illicit drug use in past month, }\end{array}$ & $\begin{array}{l}\text { Marijuana: } \mathrm{HO} \text { men } 0.7, \mathrm{HO} \text { women } 0.8 \text {, all nss, } \mathrm{B} \\
\text { men } 1.3 \mathrm{nss}, \mathrm{B} \text { women } 2.8 \mathrm{ss} \text {. } \\
\text { Drug use: } \mathrm{HO} \text { men } 1.6, \mathrm{HO} \text { women } 1.2, \mathrm{~B} \text { men } \\
\text { 2.2, B women } 3.4\end{array}$ & $\begin{array}{l}\text { B students had clearly } \\
\text { larger SO differences than } \\
\text { HO students. }\end{array}$ & $\begin{array}{l}\text { Comparable for } \\
\mathrm{HO}, \text { but for B, } \\
\text { larger effects for } \\
\text { women }\end{array}$ & \\
\hline $\begin{array}{l}\text { Gilman et al. } \\
\text { (2001) }\end{array}$ & $\begin{array}{l}4,910 \text { youth and adults (15-54 } \\
\text { years-old), USA, random } \\
\text { sample, National Comorbidity } \\
\text { Survey (NCS) 1990-1992 }\end{array}$ & $\begin{array}{l}2.5 \% \text { SM, } 1.0 \% \text { had "sexual } \\
\text { intercourse" only with persons of } \\
\text { the same sex in the past } 5 \text { years, } \\
1.6 \% \text { with both men and women, } \\
\text { Interview }\end{array}$ & $\begin{array}{l}\text { Mental disorders (DSM-III-R), } \\
\text { lifetime and past year, assessed } \\
\text { with the CIDI, SA with single } \\
\text { items for past year and lifetime }\end{array}$ & $\begin{array}{l}\text { 12-Month (women, men) } \\
\text { GAD: } 3.9 \text { nss, } 0.8 \text { nss, PD: } 2.1 \text { nss, } 1.1 \text { nss, } \\
\text { PTSD } 4.4 s s, 0.7 n s s, \text { MD: } 3.7 \text { ss } 1.6 \text { nss, AD: } 4.4 \\
\text { ss, } 0.9 \text { nss, DD: } 2.0 \text { ss, } 2.6 \text { ss, SA: n.a., } 2.5 \text { ss. } \\
\quad \text { Lifetime } \\
\text { GAD: } 3.2 \text { ss, } 2.8 \text { nss, PD: } 2.6 \text { nss, } 1.2 \text { nss, } \\
\text { PTSD } 2.7 \text { ss, } 1.1 \text { nss, MD: } 1.9 \text { ss, } 1.5 \text { nss, AD: } 2.2\end{array}$ & None reported & $\begin{array}{l}\text { Larger SO } \\
\text { differences for } \\
\text { women }\end{array}$ & $\begin{array}{l}\text { Low power, } \\
\text { Sexual } \\
\text { intercourse } \\
\text { may be } \\
\text { inappropriate } \\
\text { for SM }\end{array}$ \\
\hline
\end{tabular}




\begin{tabular}{|c|c|c|c|c|c|c|c|}
\hline & & & & nss, 1.4 nss, DD: $1.7,2.2$ ss, SA: 1.5 nss, 2.4 nss & & & \\
\hline $\begin{array}{l}\text { Grant et al. } \\
(2014)\end{array}$ & $\begin{array}{l}\text { 2,011 students, } 18-58 \text { years- } \\
\text { old (mean 22.6) from a large } \\
\text { Midwestern University, USA, } \\
\text { 2011, random sample from } \\
\text { enrollment list. }\end{array}$ & $\begin{array}{l}5.6 \% \text { identified as LGB or queer, } Q \\
\text { were excluded, } \\
\text { Web-survey }\end{array}$ & $\begin{array}{l}\text { Minnesota Impulsive Disorders } \\
\text { Interview (MIDI), depressive } \\
\text { symptoms with the PHQ-9, } \\
\text { perceived stress scale for stress } \\
\text { in past month, lifetime diagnosis } \\
\text { of MD and social anxiety. }\end{array}$ & $\begin{array}{l}\text { Strong evidence for depression }(B F=13.9) \text { and } \\
\text { stress (BF 11.7) } \\
\text { ss larger risk for diagnosis: } \\
\text { MD (2.6) } \\
\text { social anxiety disorder }(4.0)\end{array}$ & None reported & None reported & \\
\hline $\begin{array}{l}\text { Gruskin \& } \\
\text { Gordon (2006) }\end{array}$ & $\begin{array}{l}12,729 \text { adults, USA, mean age } \\
42 \text { years-old, stratified random } \\
\text { sample, } 1999 \text { and } 2002, \text { from } \\
\text { members of the Kaiser } \\
\text { Permanente Medical Care } \\
\text { Program, representative of the } \\
\text { non-Medicaid insured adults } \\
\text { in Northern California }\end{array}$ & $\begin{array}{l}1.6 \% \text { identified as } \mathrm{L} \text { and } 3.4 \% \text { as } \\
\text { gay men. B orientation was only } \\
\text { solicited in the } 2002 \text { survey and } \\
\text { was excluded to allow pooling. } \\
\text { Self-administered questionnaire }\end{array}$ & $\begin{array}{l}\text { Items on current/past smoking, } \\
\text { heavy drinking (typical } \geq 4 \\
\text { drinks for women and } \geq 5 \text { for } \\
\text { men in one occasion). } \\
\text { Items on feeling depressed } \geq 2 \\
\text { weeks in past year or taking } \\
\text { antidepressants, mental distress } \\
\text { most of the time in past year. }\end{array}$ & $\begin{array}{l}\text { Effects for men, women } \\
\text { Smoking: men } 1.6, \text { women } 2.4 \text {, all ss. } \\
\text { Drinking: } 1.5,2.1 \text {, all ss. } \\
\text { Depressed/Depressants: } 3.9 \mathrm{ss}, 1.5 \mathrm{ss} \\
\text { Distress: } 1.5 \mathrm{ss}, 1.2 \mathrm{nss}\end{array}$ & None reported & $\begin{array}{l}\text { Larger effects } \\
\text { among women for } \\
\text { smoking, } \\
\text { But reversed for } \\
\text { depression and } \\
\text { distress. }\end{array}$ & \\
\hline $\begin{array}{l}\text { Herrell et al. } \\
\text { (1999) }\end{array}$ & $\begin{array}{l}\text { 3,328 adult male twin-pairs } \\
\text { from the Vietnam Era Twin } \\
\text { Registry, Hines, III, USA }\end{array}$ & $\begin{array}{l}1.8 \% \text { had a sexual relation with } \\
\text { men since the age } 18.103 \text { pairs } \\
\text { were discordant in their SO. } \\
\text { Telephone interview }\end{array}$ & Lifetime SA with single item & $\begin{array}{l}\text { SM men in discordant pairs had more SA }(6.5 \text {, } \\
\text { ss), similar result in whole sample }(7.5, \mathrm{ss}) \text {. }\end{array}$ & None reported & - & $\begin{array}{l}\text { Excellent } \\
\text { matching }\end{array}$ \\
\hline $\begin{array}{l}\text { Husky, } \\
\text { Guignard, } \\
\text { Beck, \& Michel } \\
\text { (2013) }\end{array}$ & $\begin{array}{l}27,653 \text { mostly adults }(10 \%> \\
20 \text { years-old), France, } \\
\text { Household random sample }\end{array}$ & $\begin{array}{l}\text { Not stated, } 1.2 \% \text { classified as LGB } \\
\text { and } 15.2 \% \text { as having no sexual } \\
\text { activity } \\
\text { Telephone survey, }\end{array}$ & $\begin{array}{l}\text { SA lifetime and in past year - } \\
\text { only lifetime results given. }\end{array}$ & $\begin{array}{l}\text { RR } 4.0 \text { for men and } 2.8 \text { for women. } \\
\text { Adjusted OR } 3.0 \text { for men (nss) and } 0.9 \text { for women } \\
\text { (nss, but } 2.5 \% \text { for LGB vs. } 0.9 \% \text { for HET). }\end{array}$ & & & $\begin{array}{l}\text { ORs adjusted } \\
\text { for marriage in } \\
\text { paper, thus RR } \\
\text { calculated. }\end{array}$ \\
\hline $\begin{array}{l}\text { Jorm, Korten, } \\
\text { Rodgers, } \\
\text { Jacomb, \& } \\
\text { Christensen } \\
\text { (2002) }\end{array}$ & $\begin{array}{l}4,824 \text { adults, two age groups } \\
\text { (20-24 and } 40-44 \text { years-old), } \\
\text { Australia, electoral rolls, } \\
\text { Canberra (PATH Through Life } \\
\text { Project) }\end{array}$ & $\begin{array}{l}1.0 \% \text { of men and } 1.8 \% \text { of women } \\
\text { aged } 20-24 \text { identified as HO (1.6 } \\
\text { and } 2.0 \% \text { among those aged } 40- \\
44, \text { respectively). } \\
1.8 \% \text { of men and } 2.7 \% \text { of women } \\
\text { aged } 20-24 \text { identified as B }(0.8 \% \\
\text { of men and women aged } 40-44) \text {. } \\
\text { Questionnaire, delivered by at- } \\
\text { home visits of interviewers. }\end{array}$ & $\begin{array}{l}\text { Anxiety and depression } \\
\text { symptoms (Goldberg scale), } \\
\text { alcohol use (AUDIT), emotional } \\
\text { well-being with PANAS scale }\end{array}$ & $\begin{array}{l}\text { Elevated levels (ss, effect sizes n.a.) for all mental } \\
\text { health variables (anxiety, depression, alcohol } \\
\text { use), and for both bisexual and homosexual } \\
\text { groups. Only exception: no effect for levels of } \\
\text { alcohol misuse among younger HO. }\end{array}$ & $\begin{array}{l}B \text { had poorer mental health } \\
\text { than the HO group }\end{array}$ & None discussed & \\
\hline $\begin{array}{l}\text { Julien, Jouvin, } \\
\text { Jodoin, } \\
\text { L'Archeveque, } \\
\text { \& Chartrand } \\
\text { (2008) }\end{array}$ & $\begin{array}{l}8,875 \text { sexually active adult } \\
\text { women, Quebec Health } \\
\text { Survey } 1998, \text { representative } \\
\text { random survey }\end{array}$ & $\begin{array}{l}2 \% \text { were classified as SM based } \\
\text { on their sexual behavior }(1 \% \mathrm{HO} \text {, } \\
1 \% \text { B). Each SM mother was } \\
\text { matched to a HET mother based } \\
\text { on age, income, residence, same } \\
\text { procedure for nonmothers. }\end{array}$ & $\begin{array}{l}\text { Current psychological distress } \\
\text { was assessed with the } \\
\text { EMMDP23. } \\
\text { \# times seriously drunk in past } \\
\text { year and illicit drug use in past } \\
\text { year (any) }\end{array}$ & $\begin{array}{l}\text { Ss effect for SO but not motherhood-status in } \\
\text { ANOVA. } \\
\text { Distress: among mothers } d=0.5 \\
\text { Among nonmothers: } d=0.3 \\
\text { Ss effect for both alcohol/drug use (RR 2.0, ss in } \\
\text { ANOVA). }\end{array}$ & None reported & & \\
\hline
\end{tabular}




\begin{tabular}{|c|c|c|c|c|c|c|}
\hline & & Self-administered questionnaire & & & & \\
\hline $\begin{array}{l}\text { McCabe, Boyd, } \\
\text { Hughes, \& } \\
\text { d'Arcy (2003) }\end{array}$ & $\begin{array}{l}\text { 3,571 undergraduates, age } \\
\text { n.a., from the University of } \\
\text { Michigan, USA. } \\
\text { Random sample }\end{array}$ & $\begin{array}{l}3.3 \% \text { identified as LGB }(75 \% \text { of } \\
\text { SM women and } 30 \% \text { of SM men } \\
\text { identified as B). } \\
\text { Internet- and questionnaire survey }\end{array}$ & $\begin{array}{l}\text { Alcohol and illicit drug use with } \\
\text { different items. } \\
\text { Detailed analysis for women } \\
\text { subsample see McCabe et al. } \\
\text { (2004), for ecstacy-use see } \\
\text { Boyd et al. (2003). }\end{array}$ & $\begin{array}{l}\text { Women: } \\
\text { Effects for nearly all indicators except drinking } \geq 4 \\
\text { drinks per occasion ( } 0.8 \mathrm{nss} \text { ), ranging from } 1.2 \\
\text { for heavy episodic drinking to } 4.2 \text { for alcohol use } \\
\text { past year, and ss for past year smoking ( } 3.6 \text { ), } \\
\text { marijuana use before college (1.9), marijuana use } \\
\text { in past month (2.7) or past year (3.9), ecstasy use } \\
\text { past year (3.8), illicit drug use the past year (4.8). } \\
\quad \text { Men } \\
\text { Reversed effects for heavy drinking ( } 0.4 \text { ss) and } \\
\geq 4 \text { drinks per occasion (0.6, nss). For all other } \\
\text { variables,effects ranging from } 1.1 \text { nss for } \\
\text { marijuana use before college to } 6.9 \text { ss for ecstasy } \\
\text { use before college and were ss for marijuana use } \\
\text { in past year (2.0) and past month (4.5) and past } \\
\text { year (3.1), but not illicit drug use (n.a.) }\end{array}$ & $\begin{array}{l}\text { No differences calculated } \\
\text { due to lacking power }\end{array}$ & $\begin{array}{l}\text { Slightly larger SO } \\
\text { differences for } \\
\text { alcohol and } \\
\text { marijuana use } \\
\text { among women, } \\
\text { reversed gender } \\
\text { difference for } \\
\text { ecstasy use. }\end{array}$ \\
\hline $\begin{array}{l}\text { Mercer et al. } \\
(2007)\end{array}$ & $\begin{array}{l}6,399 \text { women, } 16-44 \text { years- } \\
\text { old, UK Natsal } 2000 \text { survey, } \\
\text { stratified probability sample } \\
\text { survey of the general } \\
\text { population }\end{array}$ & $\begin{array}{l}+++ \\
4.9 \% \text { classified as } \mathrm{HO} \text { or B based } \\
\text { on } \mathrm{HO} \text { behavior (lifetime) } \\
\text { Face-to-face interviews and CASI. }\end{array}$ & $\begin{array}{l}\text { Smoking status, (non/ former } \\
\text { smoker vs. light/heavy smoker), } \\
\text { alcohol ( } \geq 15 \text { units weekly), } \\
\text { injecting drugs (ever, past } 5 \\
\text { years, past year) }\end{array}$ & $\begin{array}{l}\text { Smoking status: HO } 1.2 \text { nss, B } 2.0 \text { ss } \\
\text { Alcohol: HO } 1.6 \text { nss, B } 2.0 \text { ss } \\
\text { Injecting drugs (n.a.for HO) B: ever } 10.0 \text { ss, past } 5 \\
\text { years } 11.3 \text { ss, past year } 24.6 \text { ss }\end{array}$ & $\begin{array}{l}\text { Somewhat larger effects for } \\
\text { smoking and alcohol, } \\
\text { n.a. for drugs. }\end{array}$ & - \\
\hline $\begin{array}{l}\text { Lhomond \& } \\
\text { Saurel- } \\
\text { Cubizolles } \\
(2006)\end{array}$ & $\begin{array}{l}\text { 6,970 adults women, 20-59 } \\
\text { years old, France. } \\
\text { National Survey on Violence } \\
\text { against Women in France) } \\
\text { Representative sample }\end{array}$ & $\begin{array}{l}1.1 \% \text { classified as SM based on } \\
\text { lifetime same-sex sexual behavior. } \\
\text { Telephone interview }\end{array}$ & $\begin{array}{l}\text { Smoking, items on drinking } \\
\text { behavior, past year and lifetime } \\
\text { marijuana use and drug use. } \\
\text { High psychological distress } \\
\text { (General Health Questionnaire } \\
\text { score } \geq 5 \text { ) } \\
\text { SA (past year, lifetime) }\end{array}$ & $\begin{array}{l}\text { Smoking ( } 1.3 \text { nss). } \\
\text { Substance use, all ss: drinking a lot in difficult } \\
\text { moments } 6.2 \text {, believing drinking should be } \\
\text { reduced } 4.3 \text {, marijuana past year } 12.7 \text { (lifetime } \\
\text { 13.8), other illicit drug use past year } 9.3 \text { (lifetime } \\
\text { 9.2); high distress (1.3, nss) } \\
\text { Lifetime SA } 2.9 \text { ss, past year SA } 28.1 \text { ss }\end{array}$ & None reported & - \\
\hline $\begin{array}{l}\text { Lhomond, } \\
\text { Saurel- } \\
\text { Cubizolles, \& } \\
\text { Michaels } \\
(2014)\end{array}$ & $\begin{array}{l}\text { 9,622 adults, } 18-69 \text { years-old, } \\
\text { France, } \\
\text { Random sample }\end{array}$ & $\begin{array}{l}+++ \\
3.5 \% \text { of men and women had any } \\
\text { same sex sexual contacts, } 1.3 \% \text { of } \\
\text { women identified as LB and } 2.1 \% \\
\text { of men as GB; } 4.9 \% \text { of women and } \\
2.0 \% \text { of men were somewhat } \\
\text { attracted to the same sex. } \\
\text { Telephone survey }\end{array}$ & $\begin{array}{l}\text { Items on problematic alcohol } \\
\text { use in past year based on } \geq 2 \\
\text { positive responses to the CAGE } \\
\text { scale; past year cannabis use, } \\
\text { lifetime other illegal drug use, } \\
\text { having been depressed in past } \\
\text { year (single item). }\end{array}$ & 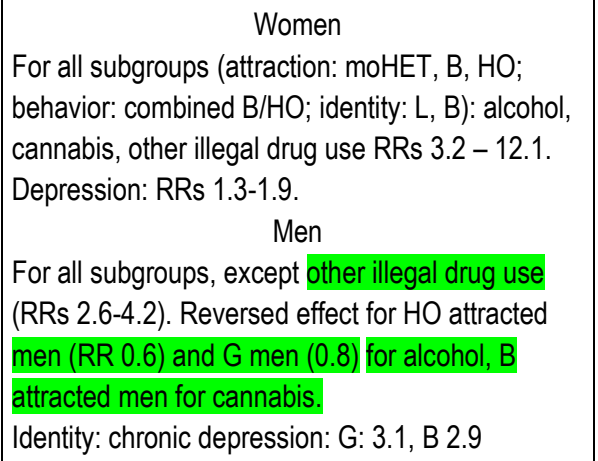 & $\begin{array}{l}\text { Among women, } \\
\text { comparable effects for B } \\
\text { and L (identity) and B, HO, } \\
\text { and moHET (attraction). } \\
\text { Among men and for } \\
\text { substance abuse, slightly } \\
\text { larger effects among } \\
\text { moHET attracted but not B } \\
\text { attracted men and for B } \\
\text { identified men. }\end{array}$ & $\begin{array}{l}\text { For substance } \\
\text { abuse, } \\
\text { substantially } \\
\text { larger effects } \\
\text { among women } \\
\text { than men. } \\
\text { For depression } \\
\text { and psychotropic } \\
\text { drug use similar } \\
\text { effects. }\end{array}$ \\
\hline
\end{tabular}


Online supplemental material to Plöderl \& Tremblay (2015). Mental health of sexual minorities. A systematic review. International Review of Psychiatry. http://dx.doi.org/10.3109/09540261.2015.1083949

\begin{tabular}{|c|c|c|c|c|c|c|c|}
\hline & & & & $\begin{array}{l}\text { depression past year: G 2.3, B } 1.7 \\
\text { Attraction: Chronic depr:. HO 3.3, B 6.0, moHET } \\
\text { 1.3, Depr. p. year: HO 2.3, B 1.7, moHET 1.3 }\end{array}$ & & & \\
\hline $\begin{array}{l}\text { King \& } \\
\text { Nazareth } \\
(2006)\end{array}$ & $\begin{array}{l}1479 \text { adults, } 18-75 \text { years-old } \\
\text { (mean 31.4-35.9, depending } \\
\text { on subgroup), UK, London, } \\
\text { consecutive people attending } \\
\text { their primary care physicians } \\
\text { over 4-8 weeks in each } \\
\text { practice }\end{array}$ & $\begin{array}{l}+++ \\
14.1 \% \text { of men }(5.3 \% \text { B and } 8.76 \% \\
\text { G), and } 10.6 \% \text { of women }(8.1 \% \text { B } \\
\text { and } 2.5 \% \text { L) stated other than } \\
\text { entirely HET experience (Likert } \\
\text { type item from entirely HET to } \\
\text { entirely HO) }\end{array}$ & $\begin{array}{l}\text { Psychological distress with the } \\
\text { General health questionnaire } \\
\text { (cutoff } \geq 3 \text { ); CAGE alcohol } \\
\text { abuse questionnaire (cutoff } \geq 2 \text { ); } \\
\text { current smoking status }\end{array}$ & $\begin{array}{l}\text { Men (G, B) } \\
\text { Smoking: } 1.6 \mathrm{nss}, 2.4 \mathrm{ss} \text {, distress: } 2.6 \mathrm{ss}, 0.7 \mathrm{nss} \text {, } \\
\text { alcohol } 1.1 \mathrm{nss}: 1.8 \mathrm{nss}, \\
\text { Women (L, B) } \\
\text { Smoking: } 2.4 \mathrm{ss}, 2.3 \mathrm{ss} \text {, distress: } 1.1 \mathrm{nss}, 1.3 \mathrm{nss} \\
\text { alcohol: } 2.1 \mathrm{nss}, 3.3 \mathrm{ss}\end{array}$ & $\begin{array}{l}\text { Among men, } B \text { less } \\
\text { distressed than } G \text {, } \\
\text { Among women comparable } \\
\text { effects for } B \text { and } L \text {. }\end{array}$ & $\begin{array}{l}\text { Larger effect for } \\
\text { alcohol/smoking } \\
\text { among women. }\end{array}$ & \\
\hline $\begin{array}{l}\text { Lian, Zuo, Lou, } \\
\text { Gao, \& Cheng } \\
(2015)\end{array}$ & $\begin{array}{l}\text { Adolescents/young adults (15- } \\
24 \text { years-old), Asia, from three } \\
\text { cities (Hanoi, } n=6,363 \text {, } \\
\text { Shanghai, } n=6,299 \text {, Taipeh, } \\
n=4,354 \text { ) }\end{array}$ & $\begin{array}{l}\text { Identified as SM (based on } \\
\text { feelings). HET category includes } \\
\text { moHET, LGB caterogy includes B, } \\
\text { moHO, and HO. F2F interviews, } \\
\text { CASI for sensitive questions. }\end{array}$ & SA in past year & $\begin{array}{l}\text { Hanoi } 2.9 \text { nss } \\
\text { Shanghai } 2.0 \text { ss } \\
\text { Taipeh } 1.6 s s\end{array}$ & None reported & None reported & \\
\hline $\begin{array}{l}\text { Mattocks et al. } \\
\text { (2013) }\end{array}$ & $\begin{array}{l}335 \text { adult female veterans } \\
\text { (mean age n.a.), USA, } \\
\text { Womens Veterans Cohort } \\
\text { Study, 2008-2011 }\end{array}$ & $\begin{array}{l}10.4 \% \text { identified as LB (asexual or } \\
\text { Q excluded). } \\
\text { Questionnaire and electronic } \\
\text { medical record. }\end{array}$ & $\begin{array}{l}\text { Smoking (yes/no) and } \\
\text { hazardous drinking (AUDIT } \\
\text { score } \geq 8 \text { ). ICD-9 codes from } \\
\text { medical records. }\end{array}$ & $\begin{array}{l}\text { Bipolar disorder ( } 6.2 \mathrm{ss}) \text {, anxiety disorder (1.8 } \\
\text { nss), MD ( } 2.0 \mathrm{nss}), \text { PTSD (1.3 nss), any mental } \\
\text { health disorder (1.9, nss), smoking ( } 2.5, \mathrm{ss}) \text {, } \\
\text { drinking (2.4, ss). }\end{array}$ & None reported & - & \\
\hline $\begin{array}{l}\text { Pope, lonescu- } \\
\text { Pioggia, \& } \\
\text { Pope (2001) }\end{array}$ & $\begin{array}{l}796 \text { seniors of a New-England } \\
\text { Colleges, US, } 1999 \text { and } \\
\text { previous replications in 1969, } \\
\text { 1978, 1989. }\end{array}$ & $\begin{array}{l}5 \% \text { reported at least one same-sex } \\
\text { sexual contact until orgasm since } \\
\text { college age. } \\
\text { Questionnaire }\end{array}$ & $\begin{array}{l}\text { Alcohol and substance use with } \\
\text { single items. }\end{array}$ & More SM students used drugs $(2.3, s s)$. & None reported & None reported & \\
\hline $\begin{array}{l}\text { Priebe \& } \\
\text { Svedin (2012) }\end{array}$ & $\begin{array}{l}3,423 \text { high school seniors, } 16- \\
22 \text { years old (mean 18.5), } \\
\text { Sweden, } \\
\text { Youth, Sex and Internet } \\
\text { Project }\end{array}$ & $\begin{array}{l}9.5 \% \text { identified as LGBQ and } \\
15.8 \% \text { were classified as SM } \\
\text { based on current emotional/sexual } \\
\text { HO or B attraction (Likert scale } \\
\text { from } 1-5) \text {. HO attraction: low score } \\
(<3) \text { on the opposite sex scale and } \\
\text { a high score }(\geq 3) \text { on the same-sex } \\
\text { scale. B attraction: high score }(\geq 3) \\
\text { on both scales. Asexual: low } \\
\text { score on both scales (<3). SM } \\
\text { consisted of HO, B, and asexual } \\
\text { attracted. }\end{array}$ & SCL-25 & $\begin{array}{l}\text { For identity, ss effects among both victimized and } \\
\text { non-victimized. } \\
\text { SS effects also in multivariate regression (ORs > } \\
2.1, \text { ss). } \\
\text { Similar results for attraction and identity. }\end{array}$ & & None reported & \\
\hline $\begin{array}{l}\text { Rath, Villanti, } \\
\text { Rubenstein, \& } \\
\text { Vallone (2013) }\end{array}$ & $\begin{array}{l}\text { 4,159 adults, 18-34 years-old, } \\
\text { USA, Legacy's Young Adult } \\
\text { Cohort Study 2011, }\end{array}$ & $\begin{array}{l}2.9 \% \text { identified as } \mathrm{HO}, 3.3 \% \mathrm{~B}, \\
0.3 \% \mathrm{~T}, 2.3 \% \mathrm{Q}, 1.2 \% \text { as other }(\mathrm{O})\end{array}$ & $\begin{array}{l}\text { MD (PHQ-2), GAD (GAD-2), } \\
\text { current use of alcohol, } \\
\text { marijuana, other drugs, past }\end{array}$ & $\begin{array}{l}\text { Smoking:. HO 1.6, B 1.4, T 1.5, O 0.9, Q } 0.4 \\
\text { Alcohol: HO 1.3, B 1.5, T 1.6, O 0.8, Q } 0.4 \\
\text { Marijuana: HO 1.0, B 3.3, T n.a., O 1.3, Q } 0.5\end{array}$ & $\begin{array}{l}\text { HO and B comparable. } \\
\text { T comparable for smoking } \\
\text { and alcohol but lower for }\end{array}$ & None reported & $\begin{array}{l}\text { Very few T and } \\
O \text { identified - } \\
\text { Results likely }\end{array}$ \\
\hline
\end{tabular}


Online supplemental material to Plöderl \& Tremblay (2015). Mental health of sexual minorities. A systematic review. International Review of Psychiatry. http://dx.doi.org/10.3109/09540261.2015.1083949

\begin{tabular}{|c|c|c|c|c|c|c|c|}
\hline & $\begin{array}{l}\text { representative sample of } \\
\text { households with cellphones }\end{array}$ & & month and current smoking, & $\begin{array}{l}\text { Other drugs: HO 3.8, B 4.7, T n.a., O 1.7, Q } 2.0 \\
\text { MD: HO 1.5, B 1.6, T 0.3, O 1.2, Q 2.2 } \\
\text { GAD: HO 1.4, B 1.4, T n.a., O 0.7, Q } 1.3\end{array}$ & $\begin{array}{l}\text { MD (other n.a.) } \\
\text { Smaller effects among } \\
\text { "other" defined and Q } \\
\text { (some effects reversed) }\end{array}$ & & $\begin{array}{l}\text { not } \\
\text { representative. }\end{array}$ \\
\hline $\begin{array}{l}\text { Reed, Prado, } \\
\text { Matsumoto, \& } \\
\text { Amaro (2010) }\end{array}$ & $\begin{array}{l}998 \text { young adults, majority < } \\
21 \text { years-old (likely > 18), } \\
\text { random sample from all } \\
\text { enrolled undergraduates in a } \\
\text { Boston University, } 2005 .\end{array}$ & $\begin{array}{l}4.2 \% \text { identified as LGB }(57 \% \text { of } \\
\text { them as B). } \\
\text { Web survey. }\end{array}$ & $\begin{array}{l}\text { Past month alcohol use, binge } \\
\text { drinking ( } \geq 5 \text { drinks men, } \geq 4 \\
\text { drinks women in one sitting in } \\
\text { typical week). Number of } \\
\text { different drugs and \# drug use in } \\
\text { past month. Consequences of } \\
\text { alcohol/drug use (RAPI) Index. } \\
\text { SA or suicide plans (past year. }\end{array}$ & $\begin{array}{l}\text { Alcohol } 1.5 \mathrm{nss} \\
\text { binge drinking OR } 1.0 \text {, nss } \\
\text { illicit drug use } 2.0 \mathrm{ss} \\
\text { suicide attempt/planning } 6.6 \mathrm{ss} \\
\# \text { illicit drug use past month } d=0.3 \mathrm{ss} \\
\text { \# of different drugs past month } d=0.4 \mathrm{ss} \\
\text { Consequences drug/alcohol } d=0.5 \mathrm{ss}\end{array}$ & None reported & None reported & $\begin{array}{l}\text { Also includes } \\
\text { adolescents }\end{array}$ \\
\hline $\begin{array}{l}\text { Rhodes, } \\
\text { McCoy, Wilkin, } \\
\text { \& Wolfson } \\
(2009)\end{array}$ & $\begin{array}{l}\text { 4,167 adult men, mean age } \\
20.5 \text { years-old, } 81 \% \text { white, } \\
\text { Students, North Carolina, } \\
\text { Random cross-sectional } \\
\text { sample }\end{array}$ & $\begin{array}{l}4.9 \% \text { identified as } \mathrm{G} \text { or } \mathrm{HO}, \mathrm{B}, \mathrm{T} \text {, } \\
\text { or O; only } \mathrm{G} / \mathrm{HO} \text { were analyzed. } \\
\text { Questionnaire via computer }\end{array}$ & $\begin{array}{l}\text { Past month binge drinking, } \\
\text { current alcohol use, } \\
\text { drunkenness in typical week, } \\
\text { past month smoking, marijuana, } \\
\text { methamphetamines, cocaine, } \\
\text { GHB, other drugs }\end{array}$ & $\begin{array}{l}\text { Heavy drinking (1.1, nss), alcohol use }(0.9, \mathrm{nss}) \text {, } \\
\text { smoking }(1.5, \mathrm{ss}) \text {, marijuana }(1.3, \mathrm{nss}) \text {, } \\
\text { methamphetamines }(4.3, \mathrm{ss}), \text { cocaine }(1.7, \mathrm{nss}) \text {, } \\
\text { GHB }(8.3, \mathrm{ss}), \text { other drugs }(1.9, \mathrm{ss})\end{array}$ & - & - & \\
\hline $\begin{array}{l}\text { Ridner, Frost, } \\
\text { \& LaJoie } \\
(2006)\end{array}$ & $\begin{array}{l}772 \text { adults, } 18-24 \text { years old, } \\
\text { Kentucky, USA }\end{array}$ & $\begin{array}{l}\text { 4.1\% of women identified as LB, } \\
9.3 \% \text { of men as GB. Excluded: } \\
0 \% \text { T and } 1 \% \text { Q, Web survey. }\end{array}$ & $\begin{array}{l}\text { Current smoking, drinking, } \\
\text { marijuana, see ACHA-NCHA } \\
\text { instrument }\end{array}$ & $\begin{array}{l}\text { Women: smoking }(4.9, \text { ss), drinking }(10.7, \mathrm{ss}) \text {, } \\
\text { marijuana }(4.9, \mathrm{ss}) \text {, } \\
\text { ORs close to } 1 \text { among men }\end{array}$ & None reported & $\begin{array}{l}\text { Larger effects for } \\
\text { women (zero- } \\
\text { effects for men) }\end{array}$ & \\
\hline $\begin{array}{l}\text { Said, Kypri, \& } \\
\text { Bowman } \\
\text { (2013) }\end{array}$ & $\begin{array}{l}\text { 6,379 young adults, mean } \\
26.5 \text { years-old, Australia, } \\
\text { students enrolled in University } \\
\text { of Newcastle, } 2010 .\end{array}$ & $\begin{array}{l}\text { Classified as } \mathrm{HO}(6.1 \%) \text { or } \mathrm{B} \\
(2.5 \%), \text { not further specified. } \\
\text { Web Survey }\end{array}$ & $\begin{array}{l}\text { Current disorders with the } \\
\text { Patient Health Questionnaire } \\
\text { (PHQ): Depression, Anxiety } \\
\text { Disorder. Eating Disorder } \\
\text { according to DSM IV criteria, } \\
\text { harmful drinking with the AUDIT. }\end{array}$ & $\begin{array}{l}\text { Depression: HO } 2.0 \text { ss, B } 4.2 \text { ss } \\
\text { Anxiety: HO } 1.8 \mathrm{ss}, \text { B } 2.4 \mathrm{ss} \\
\text { Eating disorder: HO } 1.4 \mathrm{nss}, \mathrm{B} 0.9 \mathrm{nss} \\
\text { Harmful drinking: HO } 1.9 \mathrm{ss}, \mathrm{B} 1.7 \mathrm{ss}\end{array}$ & $\begin{array}{l}\text { Larger effect among B than } \\
\text { among } \mathrm{HO} \text { for depression } \\
\text { and anxiety, but reversed } \\
\text { for Eating disorder and } \\
\text { harmful drinking. }\end{array}$ & None reported & \\
\hline $\begin{array}{l}\text { Schauer, Berg, } \\
\text { \& Bryant } \\
(2013)\end{array}$ & $\begin{array}{l}\text { 3,892 young adults, mean age } \\
\text { ca. } 23 \text { years-old, college } \\
\text { students, USA, random } \\
\text { sample of college students } \\
\text { from six colleges in } \\
\text { southeastern Universities, }\end{array}$ & $\begin{array}{l}+++ \\
6.4 \% \text { identified as } \mathrm{HO}(2.9 \%) \text { or B } \\
(3.5 \%) \text {. } \\
\text { Web survey. }\end{array}$ & $\begin{array}{l}\text { Depressive symptoms with the } \\
\text { Patient Health Questionnaire } \\
\text { (PHQ-2). } \\
\text { \# of past month drinking, } \\
\text { marijuana, smoking, and binge } \\
\text { drinking, all dichotomized } \\
\text { (yes/no), and a summary } \\
\text { measure of all those } \\
\text { dichotomized items. }\end{array}$ & $\begin{array}{l}\text { Depression PHQ-2 (Cohen's d): Men: HO } 0.2 \text {, B } \\
\text { 0.7; Women: HO 0.1, B 0.4 } \\
\text { Substance abuse index: (Cohen's d): Men: HO - } \\
\text { 0.1, B 0.2; Women: HO 0.4, B } 0.5 \\
\text { Other variables (all ORs): } \\
\text { Men (HO, B): alcohol 1.6, 1.5), binge drinking } 0.7 \text {, } \\
\text { 1.3, marijuana } 1.3,1.5 \text {, smoking } 0.7,1.2 \text {, any } \\
\text { substance } 0.8,0.9 \text {. } \\
\text { Women (HO, B): alcohol } 1.7 \mathrm{nss}, 2.1 \mathrm{ss} \text {, binge } \\
\text { drinking } 1.6 \mathrm{nss}, 1.8 \mathrm{ss} \text {, marijuana } 3.0 \mathrm{nss}, 3.4 \mathrm{ss} \text {, } \\
\text { smoking } 1.5 \mathrm{nss}, 2.6 \mathrm{ss} \text {, any subst } 1.9 \mathrm{ss}, 2.2 \mathrm{ss}\end{array}$ & $\begin{array}{l}\text { B had ss higher depression } \\
\text { than } \mathrm{HO} \text {. } \\
\text { For substance abuse index } \\
\text { and individual substance } \\
\text { use variables, somewhat } \\
\text { larger effect for B than } \mathrm{HO} \text {. }\end{array}$ & $\begin{array}{l}\text { Small and } \\
\text { sometimes } \\
\text { reversed effect for } \\
\text { substance use for } \\
\text { men but not } \\
\text { women. }\end{array}$ & \\
\hline
\end{tabular}


Online supplemental material to Plöderl \& Tremblay (2015). Mental health of sexual minorities. A systematic review. International Review of Psychiatry. http://dx.doi.org/10.3109/09540261.2015.1083949

\begin{tabular}{|c|c|c|c|c|c|c|c|}
\hline \begin{tabular}{|l|} 
Skegg, Nada- \\
Raja, Dickson, \\
Paul, \& \\
Williams (2003)
\end{tabular} & $\begin{array}{l}942 \text { young adults, 26-years- } \\
\text { old, New Zealand, birth cohort } \\
\text { study, Dunedin } \\
\text { Multidisciplinary Health } \\
\text { and Development Study }\end{array}$ & $\begin{array}{l}11 \% \text { of men and } 26 \% \text { of women } \\
\text { had other than exclusive HET } \\
\text { attraction (currently or sometimes } \\
\text { in the past). Among men, } 1.7 \% \\
\text { had persistent } \mathrm{HO} \text { attraction, } 9.4 \% \\
\text { minor } \mathrm{HO} \text { attraction (1.9\% and } \\
23.8 \% \text { among women, } \\
\text { respectively). Computer based } \\
\text { questionnaire (SO) and interview } \\
\text { (psychiatric symptoms) }\end{array}$ & $\begin{array}{l}\text { Depressed mood and substance } \\
\text { abuse in the past year with } \\
\text { single items. } \\
\text { SA (self harm with and without } \\
\text { intent to die) in lifetime. }\end{array}$ & $\begin{array}{l}\text { Men (persistent HO, B). } \\
\text { Depressive mood: } 1.1 \text { nss, } 2.6 \mathrm{ss} \\
\text { Substance abuse: } 0.8 \mathrm{nss}, 3.3 \mathrm{ss} \\
\text { SA: } 2.9 \text { nss, } 5.3 \mathrm{nss} \\
\text { Women: } \\
\text { Depressive mood: } 4.1 \mathrm{ss}, 1.3 \mathrm{nss} \\
\text { Substance abuse: } 4.0 \mathrm{nss}, 2.8 \mathrm{ss} \\
\text { SA: } 4.9 \text { nss, } 1.2 \mathrm{nss}\end{array}$ & $\begin{array}{l}\text { Men, SO differences larger } \\
\text { for B than persistent HO for } \\
\text { depression and substance } \\
\text { use but not for SA. } \\
\text { Women, SO differences } \\
\text { larger among HO women. }\end{array}$ & $\begin{array}{l}\text { Smaller SO } \\
\text { differences for } \\
\text { women. } \\
\text { Larger SO } \\
\text { differences for B } \\
\text { compared to HO } \\
\text { men (except SA), } \\
\text { reversed effect } \\
\text { among women. }\end{array}$ & \\
\hline $\begin{array}{l}\text { Talley, Sher, } \\
\text { Steinley, } \\
\text { Wood, \& } \\
\text { Littlefield } \\
\text { (2012) }\end{array}$ & $\begin{array}{l}2,068 \text { young adults, first year } \\
\text { college students, mean age } \\
18.8 \text { years-old (SD } 0.47) \text {, } \\
\text { followed yearly over the next } 5 \\
\text { years. }\end{array}$ & $\begin{array}{l}+++ \\
11.0,13.2,13.1,13.0 \% \text { (year 2-5 } \\
\text { of survey) identified as SM } \\
\text { (moHET, B, moHO, HO), } \\
4.5,6.1,5.4,7.5 \% \text { were SM based } \\
\text { on behavior (general, no time } \\
\text { specified) and 10.3, 12.4, 14.3, } \\
17.1 \% \text { based on attraction with } \\
\text { similar 7-scaled items. } \\
\text { Latent class analysis with all three } \\
\text { SO dimensions: women: } 1.6 \% \\
\text { HO/B, } 9.7 \text { moHET, } 27.2 \% \\
\text { increasingly becoming moHET } \\
\text { (inc.moHET) over time. For men } \\
\text { only two SM classes: } 3.2 \% \text { HO/B, } \\
16.0 \% \text { and inc.moHET emerged. } \\
\text { Paper \& Pencil questionnaire at } \\
\text { first assessment, then web } \\
\text { surveys. }\end{array}$ & $\begin{array}{l}\text { Frequency of alcohol use in past } \\
3 \text { months, typical \# drinks when } \\
\text { drinking, binge drinking, } \\
\text { \# being drunken in past month, } \\
37 \text { consequences of alcohol in } \\
\text { past month. }\end{array}$ & $\begin{array}{l}\text { Women: } \\
\text { In first year, moHET ss more alcohol quantity and } \\
\text { binge drinking (but decrease in second year). No } \\
\text { ss effects for drunkenness. } \\
\text { Inc.moHET and moHET had more neg. } \\
\text { consequences across all years, also compared to } \\
\text { HO/B. } \\
\text { Men: } \\
\text { Ss reversed effect for inc.moHET over the years } \\
\text { for alcohol quantity but they had elevated neg. } \\
\text { consequences. } \\
\text { Effect sizes n.a. }\end{array}$ & $\begin{array}{l}\text { MoHET women more at } \\
\text { risk, but mixed finding } \\
\text { among moHET males (less } \\
\text { at risk for alcohol quantity } \\
\text { but more for negative } \\
\text { consequences of drinking). }\end{array}$ & None reported & $\begin{array}{l}\text { Special SO } \\
\text { variable } \\
\text { created from } \\
\text { latent class } \\
\text { analyses }\end{array}$ \\
\hline $\begin{array}{l}\text { Ueno (2010a) } \\
\text { "Mental Health } \\
\text { Differences } \\
\text { between } \\
\text { Young Adult..." }\end{array}$ & $\begin{array}{l}1,492 \text { young adults } \\
\text { recontacted from previous } \\
\text { Adolescent Transitions Study } \\
\text { carried out in Miami Dade } \\
\text { County, } 9 \% \text { between } 19-21 \\
\text { years-old, random sample with } \\
\text { corrections made. }\end{array}$ & $\begin{array}{l}4.3 \% \text { classified as SM based on } \\
\text { having reported less that } 100 \% \\
\text { opposite-sex sexual partners in the } \\
\text { past Transitions Study, meaning } \\
\text { all present study mental health } \\
\text { results represents post 'having had } \\
\text { same-sex partner(s)' outcomes. }\end{array}$ & $\begin{array}{l}\text { Depression with the CES-D, } \\
\text { summary score of past month } \\
\text { different drug use. }\end{array}$ & $\begin{array}{l}\text { Ss effects for depression } d=0.7 \text { and } \\
\text { drug use } d=0.7\end{array}$ & Not reported & Note reported & \\
\hline \begin{tabular}{|l|} 
Wang, \\
Hausermann, \\
Wydler, \\
Mohler-Kuo, \& \\
Weiss (2012)
\end{tabular} & $\begin{array}{l}22,191 \text { males, mostly } 20 \\
\text { years-old, } \\
\text { Second Swiss Recruit Survey } \\
\text { on health (ch-x, 2002, 03); } \\
50 \% \text { of compulsory recruits }\end{array}$ & $\begin{array}{l}\text { SM based on attraction, see Wang } \\
\text { et al. (2014). Results reported for } \\
\text { "HO/B" (1.6\%) vs. "only HET". } \\
91.7 \% \text { only HET, } 5.3 \% \text { moHET, } \\
0.9 \% \text { B, } 0 \% \text { HO, } 0.7 \% \text { only HO, }\end{array}$ & SA lifetime & SA, OR: 4.1, ss. & Not reported & - & \\
\hline
\end{tabular}


Online supplemental material to Plöderl \& Tremblay (2015). Mental health of sexual minorities. A systematic review. International Review of Psychiatry. http://dx.doi.org/10.3109/09540261.2015.1083949

\begin{tabular}{|c|c|c|c|c|c|c|c|}
\hline & surveyed & $\begin{array}{l}\text { and } 1.2 \% \text { non-response. } \\
\text { Questionnaires }\end{array}$ & & & & & \\
\hline $\begin{array}{l}\text { Wang et al. } \\
(2014)\end{array}$ & $\begin{array}{l}5,990 \text { young adult men, } \\
\text { Switzerland, recruited form } \\
\text { mandatory evaluations for } \\
\text { military service and additional } \\
\text { voluntary study participation. }\end{array}$ & $\begin{array}{l}+++ \\
8.6 \% \text { were classified as SM based } \\
\text { on some degree of } \mathrm{HO} \text { attraction } \\
(5.6 \mathrm{moHET}, 1.1 \% \mathrm{~B}, 0.7 \% \mathrm{moHO}, \\
1.1 \% \mathrm{HO}) \text {. } \\
\text { Questionnaire }\end{array}$ & $\begin{array}{l}\text { Mental health in past month with } \\
\text { the MOS-SF } 12 \text { scale, } \\
\text { MD in past week with the MDI, } \\
\text { past year ADHD with the ASRS- } \\
\text { V1.1 scale from the CIDI, } \\
\text { lifetime antisocial personality } \\
\text { disorder from the MINI. Past } \\
\text { year SA with a single item. }\end{array}$ & $\begin{array}{l}\text { Effects in this order: moHET, B, moHO, HO } \\
\text { MD: } 1.6 \mathrm{nss}, 2.8 \mathrm{ss}, 8.7 \mathrm{ss}, 4.5 ; \\
\text { ADHD: } 2.0 \mathrm{ss}, 1.2 \mathrm{nss}, 1.9 \mathrm{nss}, 3.5 \mathrm{ss} \text {; } \\
\text { Antisocial PD: } 1.9 \mathrm{ss}, 0.9 \mathrm{ss}, \mathrm{HO} 1.3 \mathrm{nss}, 0.6 \mathrm{nss} \\
\text { SA: } 2.2 \mathrm{ss}, 8.3 \mathrm{ss}, 2.0 \mathrm{nss}, \mathrm{HO} 4.1\end{array}$ & $\begin{array}{l}\text { Larger effects for HO than } \\
\text { B except for SA and } \\
\text { antisocial PD. }\end{array}$ & - & \\
\hline $\begin{array}{l}\text { Wichstrom \& } \\
\text { Hegna (2003) }\end{array}$ & $\begin{array}{l}2,924 \text { young adults, mean age } \\
22.1 \text { years-old, highschools. } \\
\text { Random sample, data from } \\
\text { third wave (1999) used here, } \\
\text { Young in Norway study }\end{array}$ & $\begin{array}{l}\text { Sexual orientation classified with } \\
\text { same-sex sexual contacts since } \\
\text { age } 12(6.5 \%) \text {, sexual attraction } \\
(15.5 \%) \text { and identification }(11.4 \%), \\
\text { Questionnaire }\end{array}$ & $\begin{array}{l}\text { SA lifetime (separated from } \\
\text { parasuicides, i.e., overdoses) }\end{array}$ & $\begin{array}{l}\text { Behavior: } 4.7 \text {, ss } \\
\text { Both attraction and identity: } 2.7 \mathrm{ss}\end{array}$ & None reported & $\begin{array}{l}\text { Women had } \\
\text { higher risk for } \\
\text { future SA than } \\
\text { men }\end{array}$ & \\
\hline
\end{tabular}




\section{Adolescent Samples}

\section{YOUTH RISK BEHAVIOR SURVEYS}

\begin{tabular}{|c|c|c|c|c|c|c|c|}
\hline Author (Year) & $\begin{array}{l}\text { Sample, } \\
\text { Sampling Method }\end{array}$ & $\begin{array}{l}\text { Percentage of Sexual Minority } \\
\text { Individuals, Dimensions of } \\
\text { Sexual Orientation, } \\
\text { Assessment Method }\end{array}$ & Outcome Variables & $\begin{array}{l}\text { Main Results (SM vs. HET) } \\
\text { All Effects ORs if not other specified }\end{array}$ & Subgroup Differences & \begin{tabular}{|l} 
Gender \\
Differences
\end{tabular} & Note \\
\hline $\begin{array}{l}\text { Bostwick et al. } \\
(2014)^{b+}\end{array}$ & $\begin{array}{l}72,671 \text { adolescents, US, } \\
\text { Pooled data } 2005 \text { and } 2007 \\
\text { from } 14 \text { regions in the US, }\end{array}$ & $\begin{array}{l}8.4 \% \text { were classified as SM, i.e., } \\
\text { they reported a nonheterosexual } \\
\text { identity, any same-sex behavior, or } \\
\text { any same-sex attractions }\end{array}$ & Past year SA & $\begin{array}{l}\text { Ss effect } 3.9 \\
\text { SO differences also were apparent among all } \\
\text { racial subgroups. Within SM, elevated SA rates, } \\
\text { compared to Whites, were observed among } \\
\text { Alaskan/Pacific islander (1.7 ss), Latino (1.5 ss), } \\
\text { multiracial ( } 1.2 \text { nss), and somewhat lower among } \\
\text { Asian or Black adolescents (both } 0.9, \text { nss). }\end{array}$ & None reported & $\begin{array}{l}\text { For total sample, } \\
\text { comparable SO } \\
\text { differences: boys } \\
\text { OR 4.6, girls OR } \\
3.8 \text { (based from } \\
\text { Table 2, weighted } \\
\text { data) }\end{array}$ & \\
\hline $\begin{array}{l}\text { Button, } \\
\text { O'Connell, \& } \\
\text { Gealt (2012) }\end{array}$ & $\begin{array}{l}\text { 6,636 adolescents, 11-18 } \\
\text { years-old (mostly 15-16), US, } \\
\text { Delaware, 2003, 2005, } 2007\end{array}$ & $\begin{array}{l}5.3 \% \text { identified as SM }(1.1 \% \mathrm{HO} \text {, } \\
3.1 \% \mathrm{~B}, 1.1 \% \mathrm{Q})\end{array}$ & $\begin{array}{l}\text { Substance use (any vs. none) } \\
\text { based on \# drink past month, } \\
\text { \# binge drinking, \# marijuana } \\
\text { SA (lifetime) }\end{array}$ & $\begin{array}{l}\text { Alcohol: HO } 1.1 \text { nss, B } 2.0 \text { ss, Q } 0.7 \text { nss } \\
\text { Binge drinking: HO } 1.7 \text { ss, B } 2.0 \text { ss, Q } 1.2 \text { nss } \\
\text { Marijuana: HO } 1.3 \text { nss, B } 2.7 \text { ss, Q 1.0nss } \\
\text { SA: HO 5.7, B 6.2, Q 6.4, all ss }\end{array}$ & $\begin{array}{l}\text { Larger effects for B than } \\
\text { HO for alcohol, binge } \\
\text { drinking and marijuana but } \\
\text { not SA }\end{array}$ & $\begin{array}{l}\text { None reported. } \\
\text { Within SM, no ss } \\
\text { difference for } \\
\text { substance abuse. }\end{array}$ & \\
\hline $\begin{array}{l}\text { DuRant, } \\
\text { Krowchuk, \& } \\
\text { Sinal (1998) }\end{array}$ & $\begin{array}{l}\text { 3,886 male adolescents, US, } \\
\text { random sample, } \\
\text { Vermont YRBS } 1995\end{array}$ & $\begin{array}{l}8.7 \% \text { had at least one „sexual } \\
\text { intercourse“ with another male. } \\
\text { Questionnaire }\end{array}$ & $\begin{array}{l}\text { SA (past year), substance use } \\
\text { past month }\end{array}$ & $\begin{array}{l}\text { Significant correlation of \# of male sexual partners } \\
\text { and SA attempt ( } r .3) \text {, alcohol use ( } r \text {.2), Marijuana } \\
\text { (r .2), tobacco (.2) }\end{array}$ & None reported & - & $\begin{array}{l}\text { Effects only } \\
\text { given for \# of } \\
\text { HS-partners }\end{array}$ \\
\hline $\begin{array}{l}\text { Faulkner \& } \\
\text { Cranston } \\
(1998)\end{array}$ & $\begin{array}{l}1,668 \text { adolescents, mean age } \\
16 \text { years-old, USA. } \\
\text { Random sample, } \\
\text { Massachusetts YRBS } 1993\end{array}$ & $\begin{array}{l}6.4 \% \text { ever had same-sex sexual } \\
\text { contacts. } \\
\text { Questionnaire }\end{array}$ & $\begin{array}{l}\text { SA (past year), substance use } \\
\text { past month }\end{array}$ & $\begin{array}{l}\text { Tobacco use (regular smoker ever or current } \\
\text { smoker } 0.9 \text {, current regular smoker } 1.4 \text {, all nss), } \\
\text { for alcohol use in past month or current episodic } \\
\text { drinking } 1.1 \mathrm{nss} \text {, daily drinking } 58.1 \mathrm{ss}, \geq 10 \text { times } \\
\text { heavy drinking } 4.6 \mathrm{ss} \text {, past month marijuana } 1.0 \text {, } \\
\geq 40 \text { times marijuana use } 4.1 \mathrm{ss} \text {, cocaine use at } \\
\text { least one } 7.1 \text { and } \geq 10 \text { times } 21.5, \text { SA } 2.5 \text {, ss. }\end{array}$ & None reported & None reported & \\
\hline $\begin{array}{l}\text { Garofalo, Wolf, } \\
\text { Kessel, } \\
\text { Palfrey, \& } \\
\text { DuRant (1998) }\end{array}$ & $\begin{array}{l}\text { 3,365 adolescents, USA, } \\
\text { random sample, } \\
\text { Massachusetts YRBS } 1995\end{array}$ & $\begin{array}{l}\text { 3.8\% identified as LGB }(0.6 \% \text { LG } \\
\text { and } 1.9 \% \text { B) and additional } 1.5 \% \\
\text { as Q, } \\
\text { Questionnaire }\end{array}$ & $\begin{array}{l}\text { SA (past year), substance use } \\
\text { past month }\end{array}$ & $\begin{array}{l}\text { All effects ss: } \\
\text { Cigarette smoking } 2.7 \text {, alcohol use } 2.4 \text {, binge } \\
\text { drinking } 1.7 \text {, marijuana use } 2.5 \text {, cocaine use } 2.0 \text {, } \\
\text { SA } 5.1 .\end{array}$ & $\begin{array}{l}\text { None reported. } \\
\text { No substantial difference if } \\
Q \text { were included in the HET } \\
\text { group or excluded. }\end{array}$ & None reported & \\
\hline Hatzenbuehler, & 55,599 adolescents, USA, & 0.8-2.\% identified as LG, $3.0-5.4 \%$ & SA (past year) & All effects ss: & Larger SO difference for $\mathrm{B}$, & None reported & \\
\hline
\end{tabular}


Online supplemental material to Plöderl \& Tremblay (2015). Mental health of sexual minorities. A systematic review. International Review of Psychiatry. http://dx.doi.org/10.3109/09540261.2015.1083949

\begin{tabular}{|c|c|c|c|c|c|c|c|}
\hline $\begin{array}{l}\text { Birkett, Van } \\
\text { Wagenen, \& } \\
\text { Meyer }(2014)^{b+}\end{array}$ & 2005 and 2007 from 8 regions & $\begin{array}{l}\text { as } \mathrm{B}, 1.3-4.9 \% \text { as } \mathrm{Q} \text {, } \\
\text { Questionnaire }\end{array}$ & & $\begin{array}{l}\text { LG } 3.9, \\
\text { B } 5.7 \\
\text { Q } 2.4\end{array}$ & $\begin{array}{l}\text { smaller for } Q \text { compared to } \\
\text { LG }\end{array}$ & & \\
\hline $\begin{array}{l}\text { Jiang, Perry, \& } \\
\text { Hesser (2010) }\end{array}$ & $\begin{array}{l}\text { 2,210 adolescents, USA, } \\
\text { Rhode Island } 2007\end{array}$ & $10.3 \%$ Identified as LGBQ & $\begin{array}{l}\text { Having felt sad/hopeless in past } \\
\text { year, SA past year }\end{array}$ & $\begin{array}{l}\text { Feeling sad/hopeless } 3.8 \mathrm{ss,} \\
\text { SA } 6.4 \mathrm{ss}\end{array}$ & None reported & None reported & \\
\hline $\begin{array}{l}\text { Kann et al. } \\
(2011)^{b}\end{array}$ & $\begin{array}{l}418,117 \text { adolescents from } \\
\text { selected sites (states and } \\
\text { cities pooled from 2001, 2003, } \\
2005,2007,2009 \text { ). } \\
9 \text { Sites assessed sexual } \\
\text { identity, } 12 \text { sites sexual } \\
\text { behavior. }\end{array}$ & $\begin{array}{l}\text { Median over all sites: } \\
\text { Identity: } \\
7 \% \text { SM: } 1.3 \% \text { LG, } 3.7 \% \text { B, } 2.5 \% \text { Q } \\
\text { Sexual Behavior: } \\
5.8 \% \text { SM: } 2.5 \% \text { HO, } 3.3 \% \text { B, } \\
40.5 \% \text { no sex }\end{array}$ & $\begin{array}{l}\text { Having felt sad/hopeless in past } \\
\text { year, SA past year, } \\
\text { Past month cigarette use, } \\
\text { current frequent cigarette use ( } \geq \\
20 \text { days in past month), } \\
\text { past month alcohol use and } \\
\text { binge drinking (past month), } \\
\text { Past month marijuana and } \\
\text { cocaine use, } \\
\text { ever injecting illegal drug. }\end{array}$ & $\begin{array}{l}\text { All results are RR } \\
\text { Having felt sad/hopeless: identity (LG 1.7, B 2.3, } \\
\text { Q 1.5), behavior (HO 1.4, B 1.9). } \\
\text { SA: identity (LG 4.0, B 2.9, Q 2.9), behavior (HO } \\
\text { 2.3, B 3.5). } \\
\text { Cigarette use: identity (LG 2.2, B 2.3, Q 1.3), } \\
\text { behavior (HO 1.2, B 2.0) } \\
\text { Frequent cigarette use: identity (LG 3.0, B 3.2, Q } \\
\text { 1.4), behavior (HO 1.1, B 3.1). } \\
\text { Alcohol use: identity (LG 1.3, B 1.5, Q 0.9), } \\
\text { behavior (HO 1.0, B 1.2). } \\
\text { Binge drinking, identity (LG 1.3, B 1.6, Q 1.0), } \\
\text { behavior (HO 0.9, B 1.4). } \\
\text { Marijuana: identity (LG 1.6, B 1.7, Q 1.2), } \\
\text { behavior (HO 1.1, B 1.5). } \\
\text { Cocaine use: identity (LG 9.2, B 6.1, Q 6.2), } \\
\text { behavior (HO 3.2, B 5.7). } \\
\text { Injecting illegal drug: identity (LG 9.9, B 5.1, Q } \\
\text { 6.9) and behavior (HO 3.2, B 5.7). }\end{array}$ & $\begin{array}{l}\text { LG and B identified are } \\
\text { comparable (except hard } \\
\text { drug use where LG have } \\
\text { higher levels). } \\
\text { For behavior, B have larger } \\
\text { SO differences than HO. } \\
\text { Q have smaller effects than } \\
\text { HO or B, sometimes } \\
\text { reversed effect } \\
\text { Larger effects for identity } \\
\text { than behavior }\end{array}$ & $\begin{array}{l}\text { None reported } \\
\text { (only within SO } \\
\text { group gender } \\
\text { differences). }\end{array}$ & $\begin{array}{l}\text { For tabulated } \\
\text { OR/RR } \\
\text { suicidality } \\
\text { results see } \\
\text { http://people.uc } \\
\text { algary.ca/ ram } \\
\text { say/gay-more- } \\
\text { risk- } \\
\text { suicide.htm }\end{array}$ \\
\hline $\begin{array}{l}\text { Matthews, } \\
\text { Blosnich, } \\
\text { Farmer, \& } \\
\text { Adams (2014) }\end{array}$ & $\begin{array}{l}9860 \text { adolescents, USA, grade } \\
\text { 9-12, Massachusetts YRBS, } \\
\text { 2003, 2005, } 2007\end{array}$ & $\begin{array}{l}+++ \\
5.6 \% \text { were classified as SM based } \\
\text { on lifetime sexual behavior }(2.1 \% \\
\mathrm{HO}, 3.5 \% \mathrm{~B}) \text { and } 6.3 \% \text { as } \mathrm{LGBQ} \\
(1.2 \% \mathrm{LG}, 3.1 \% \mathrm{~B}, 2.1 \% \mathrm{Q})\end{array}$ & $\begin{array}{l}\text { Past month smoking and } \\
\text { methamphetamine use }\end{array}$ & $\begin{array}{l}\quad \text { Behavioral measure of SO: } \\
\text { Women: smoking HO } 1.0 \text { nss, B } 3.8 \text { ss, } \\
\text { metamphetamine HO } 1.1 \text { nss, B } 5.3 \text { ss. } \\
\text { Men: smoking: HO } 1.4 \text { nss, B } 3.5 \text { ss; } \\
\text { methamphet.: HO 1.0, nss, B 10.2, ss. } \\
\quad \text { Identification as SO: All effects ss } \\
\text { Women: smoking L 9.9, B 6.0; } \\
\text { metamphetamine G 10.4, B 6.4. } \\
\text { Men: smoking G 2.8, B 3.0, } \\
\text { methamphetamine G and B 5.1 }\end{array}$ & $\begin{array}{l}\text { For behavioral measure of } \\
\text { SO, larger effects for B } \\
\text { than HO. } \\
\text { For identification, } \\
\text { comparable or even larger } \\
\text { effects for LB than their B } \\
\text { counterparts. }\end{array}$ & $\begin{array}{l}\text { For identification } \\
\text { measure of O, } \\
\text { larger effects for } \\
\text { girls, but not so } \\
\text { for behavioral } \\
\text { dimension of SO. }\end{array}$ & \\
\hline $\begin{array}{l}\text { Mustanski, } \\
\text { Andrews, } \\
\text { Herrick, Stall, \& } \\
\text { Schnarrs } \\
(2014)^{b+}\end{array}$ & $\begin{array}{l}16977 \text { male adolescents, } \\
\text { YRBS from } 11 \text { regions } 2005 \\
\text { and } 2007\end{array}$ & $\begin{array}{l}+++ \\
7.2 \% \text { were classified as SM based } \\
\text { on lifetime sexual behavior }(4.0 \% \\
\mathrm{HO}, 3.2 \% \mathrm{~B})\end{array}$ & $\begin{array}{l}\text { Having felt sad/hopeless nearly } \\
\text { daily for two weeks and stopped } \\
\text { doing some usual activities, } \\
\text { binge drinking ( }>3 \text { days past } \\
\text { month), past month marijuana } \\
\text { use ( } \geq 20 \text { times) and cocaine }\end{array}$ & $\begin{array}{l}\text { All effects RRs } \\
\text { Sadness: HO } 1.7 \text { ss, B 2.1ss, } \\
\text { binge drinking: HO } 1.1 \text { nss, B } 1.8 \text { ss, } \\
\text { marijuana: HO } 0.6 \text { nss, B } 2.3 \mathrm{ss,} \\
\text { cocaine: HO } 3.4 \text { ss, B } 8.2 \text { ss } \\
\text { SA: HO } 3.1 \text { ss, B: } 6.7 \text { ss }\end{array}$ & $\begin{array}{l}\text { Larger effects among B } \\
\text { compared to } \mathrm{HO}\end{array}$ & - & SA with injuries \\
\hline
\end{tabular}


Online supplemental material to Plöderl \& Tremblay (2015). Mental health of sexual minorities. A systematic review. International Review of Psychiatry. http://dx.doi.org/10.3109/09540261.2015.1083949

\begin{tabular}{|c|c|c|c|c|c|c|c|}
\hline & & & $\begin{array}{l}\text { use ( } \geq 3 \text { times), past year } S A \\
\text { with injuries that had to be } \\
\text { treated by doctor/nurse. }\end{array}$ & & & & \\
\hline $\begin{array}{l}\text { Newcomb, } \\
\text { Birkett, Corliss, } \\
\& \text { Mustanski } \\
(2014)^{b+}\end{array}$ & $\begin{array}{l}49,307 \text { adolescents, } 2005 \text { and } \\
2007 \text { YRBS, from } 3 \text { cities and } \\
5 \text { states. } \\
\text { Analytic sample size varied } \\
\text { with each variable. }\end{array}$ & $\begin{array}{l}\text { Identity (GL, B) and behavior }(\mathrm{HO} \text {, } \\
\text { B) }\end{array}$ & $\begin{array}{l}\text { Any lifetime use of marijuana, } \\
\text { cocaine, inhalants, } \\
\text { methamphetamine, heroin, and } \\
\text { MDMA (separate variable for } \\
\text { each drug). }\end{array}$ & $\begin{array}{l}\text { Effects } 1.3-5.8, \text { mostly > 2, ss if not marked nss } \\
\quad \text { Identity (LG, B, Q) } \\
\text { Marijuana: } 1.4 \text { nss, } 1.7,0.8 \text { nss } \\
\text { Cocaine: } 1.8,2.0,2.2, \text { Inhalants: } 1.9,2.1,2.6 \text {, } \\
\text { Methamphetamines: } 2.1,3.5,3.0, \\
\text { Heroin: } 2.7,2.8,4.7, \text { MDMA: } 1.7,2.1,2.3 \\
\quad \text { Behavior (HO, B): } \\
\text { Marijuana: } 0.9 \text { nss, } 1.7 \text {, Cocaine: } 1.7,3.4, \\
\text { Inhalants: } 1.3 \text { nss, } 2.3 \text {, Metamphet. } 2.3,4.1 \text {, } \\
\text { Heroin: } 3.3,5.6 \text { ss, MDMA: } 1.8,3.7\end{array}$ & $\begin{array}{l}\text { Identity: effects for B } \\
\text { slightly larger than for LG. } \\
\text { Behavior: larger differences } \\
\text { of effects between B and } \\
\text { LG }\end{array}$ & None reported & \\
\hline $\begin{array}{l}\text { Olshen, } \\
\text { McVeigh, } \\
\text { Wunsch-Hitzig, } \\
\text { \& Rickert } \\
\text { (2007) }\end{array}$ & $\begin{array}{l}8080 \text { adolescents, } \geq 14 \text { years- } \\
\text { old, New York City YRBS }\end{array}$ & $\begin{array}{l}\text { +++ } \\
\text { Identity }(L G, B, Q)\end{array}$ & $\begin{array}{l}\text { SA in the past year with single } \\
\text { item }\end{array}$ & $\begin{array}{l}\text { Girls: all ss except L 0.9; B 5.3, Q 2.4; } \\
\text { Boys: all effects ss, G 6.2, B 12.8, Q } 6.8\end{array}$ & $\begin{array}{l}\text { Larger effects for B than } \mathrm{L} \\
\text { or } \mathrm{G} \text {. }\end{array}$ & $\begin{array}{l}\text { Larger effects } \\
\text { among boys than } \\
\text { girls. }\end{array}$ & \\
\hline $\begin{array}{l}\text { Pinhey \& } \\
\text { Millman (2004) }\end{array}$ & $\begin{array}{l}\text { 1,381 adolescents, grade } 9 \text { - } \\
12, \text { Guam, USA, 2001, } 95 \% \text { of } \\
\text { all high school students } \\
\text { surveyed }\end{array}$ & $\begin{array}{l}3.5 \% \text { of Boys and Girls identified } \\
\text { as LGB and } 96.5 \% \text { as HET or Q, } \\
\text { Questionnaire }\end{array}$ & $\begin{array}{l}\text { SA in the past year with single } \\
\text { item }\end{array}$ & GB boys $5.0 \mathrm{ss}$, LB girls $2.6 \mathrm{ss}$ & None reported & $\begin{array}{l}\text { Smaller SO } \\
\text { difference for } \\
\text { girls. }\end{array}$ & \\
\hline $\begin{array}{l}\text { Robin et al. } \\
(2002)\end{array}$ & $\begin{array}{l}7,458 \text { adolescents, USA, } \\
\text { Vermont YRBS } 1995+1997 \\
\text { and } 4,176 \text { youth from } \\
\text { Massachusetts YRBS } \\
1995+1997\end{array}$ & $\begin{array}{l}8 \% \text { of adolescents had same sex } \\
\text { "sexual intercourse", } \\
5 \% \text { of the Massachusetts YRBS } \\
\text { had same sex sexual contacts. } \\
\text { Questionnaire }\end{array}$ & $\begin{array}{l}\text { SA (past year) and binge } \\
\text { drinking and marijuana (past } \\
\text { month), cocaine use (lifetime) }\end{array}$ & $\begin{array}{l}\text { HO: nss effects: binge drinking } 0.9-1.0, \\
\text { marijuana 1.1-1.2, cocaine 1.1-1.8 and SA 1.5-1.7 } \\
\text { B: binge drinking 1.4-1.6, marijuana 1.9-2.3, } \\
\text { cocaine 5.4-6.1, and SA 5.7-7.8 }\end{array}$ & $\begin{array}{l}\text { Larger SO differences for } \\
\text { bisexual than compared to } \\
\text { homosexual adolescents }\end{array}$ & $\begin{array}{l}\text { No ss interactions } \\
\text { with gender }\end{array}$ & $\begin{array}{l}\text { Results from } \\
\text { Table } 1 \text { used } \\
\text { (OR in paper } \\
\text { controlled for } \\
\text { forced sex - } \\
\text { may be bias) }\end{array}$ \\
\hline $\begin{array}{l}\text { Seil, Desai, \& } \\
\text { Smith (2014) }\end{array}$ & $\begin{array}{l}\text { 8,919 adolescents from the } \\
\text { New York City YRBS }\end{array}$ & $10.8 \%$ identified as LGBQ & $\begin{array}{l}\text { Past month drinking (any in past } \\
\text { month) or marijuana, lifetime } \\
\text { other illicit drug use, past year } \\
\text { depressive mood ( } \geq 2 \text { weeks) } \\
\text { and past year SA }\end{array}$ & $\begin{array}{l}\text { All effects ss: } \\
\text { Alcohol 1.5, marijuana 2.2, other illicit drugs } 2.5 \text {, } \\
\text { depressive mood 1.9, } \\
\text { SA } 2.8\end{array}$ & None reported & None reported & \\
\hline $\begin{array}{l}\text { Shields, } \\
\text { Whitaker, } \\
\text { Glassman, } \\
\text { Franks, \& } \\
\text { Howard (2012) }\end{array}$ & $\begin{array}{l}\text { 2,154 adolescents, San } \\
\text { Francisco YRBS } 2009\end{array}$ & $\begin{array}{l}\text { ?\% identified as LGB (Q } \\
\text { excluded). }\end{array}$ & $\begin{array}{l}\text { Ever using alcohol or marijuana, } \\
\text { hard drugs (cocaine, heroin etc), } \\
\text { past year feeling depressed/sad } \\
\text { (> } 2 \text { weeks), past year SA. }\end{array}$ & $\begin{array}{l}\text { All effects ss } \\
\text { Alcohol or marijuana } 4.9 \text {, } \\
\text { hard drugs } 5.1 \\
\text { feeling depressed } 3.3 \\
\text { SA (3.6) }\end{array}$ & None reported & None reported & \\
\hline
\end{tabular}


Online supplemental material to Plöderl \& Tremblay (2015). Mental health of sexual minorities. A systematic review. International Review of Psychiatry. http://dx.doi.org/10.3109/09540261.2015.1083949

\begin{tabular}{|c|c|c|c|c|c|c|c|}
\hline $\begin{array}{l}\text { Stone et al. } \\
(2014)^{b}\end{array}$ & $\begin{array}{l}\text { YRBS, pooled from } 5 \text { different } \\
\text { locations 2001-2009 (Boston, } \\
\text { Chicago, New York City, San } \\
\text { Diego, San Francisco). }\end{array}$ & $\begin{array}{l}+++ \\
7 \% \text { of boys and } 10 \% \text { of girls } \\
\text { identified as LGBQ, and for lifetime } \\
\text { sexual behavior, } 3.0 \% \text { of boys and } \\
2.4 \% \text { of girls reported only } \mathrm{HO} \\
\text { behavior (B: } 2.0 \% \text { of boys and } \\
5.0 \% \text { of girls) }\end{array}$ & SA in past year. & $\begin{array}{l}\text { All effects ss } \\
\text { Identity } \\
\text { Girls: L 4.1, B 4.4, Q 2.3; } \\
\text { Boys: G 4.2, B 5.0, Q 3.1: } \\
\text { Behavior: } \\
\text { Girls: HO } 1.8 \text { ss, B } 2.6 \text { ss } \\
\text { Boys: HO 2.8, B } 4.1\end{array}$ & $\begin{array}{l}\text { Q identified smaller effects } \\
\text { than LG or B. } \\
\text { For identity, B and LG } \\
\text { comparable. } \\
\text { For behavior, B larger } \\
\text { effects than LG. }\end{array}$ & Comparable & $\begin{array}{l}\text { See also Kann } \\
\text { et al. (2011) }\end{array}$ \\
\hline $\begin{array}{l}\text { Stone, Luo, } \\
\text { Lippy, \& } \\
\text { Mclntosh } \\
(2014)\end{array}$ & $\begin{array}{l}3,733 \text { adolescents, } \\
\text { Milwaukee } 2007+2009\end{array}$ & $\begin{array}{l}12.4 \% \text { were SM, based on lifetime } \\
\text { sexual behavior }\end{array}$ & Past year SA & $\begin{array}{l}\text { Boys: } 3.9 \text {, ss } \\
\text { Girls: } 3.9 \text {, ss } \\
\text { White: } 8.8 \mathrm{ss,} \mathrm{Black} 3.9 \mathrm{ss,} \mathrm{Hispanic} 1.9 \text { nss, } \\
\text { Other } 3.3 \mathrm{ss}\end{array}$ & None reported & Similar & \\
\hline $\begin{array}{l}\text { Talley, } \\
\text { Hughes, } \\
\text { Aranda, Birkett, } \\
\text { \& Marshal } \\
(2014)^{+}\end{array}$ & & $\begin{array}{l}+++ \\
\text { SM status if any indicator of SO is } \\
\mathrm{HO} \text { or B (identity, behavior, } \\
\text { attraction). Identity and behavior } \\
\text { as additional variables. } \\
\text { Q and sexually inexperienced } \\
\text { were, if possible, categorized as } \\
\text { SM from other SO variables or } \\
\text { excluded instead. }\end{array}$ & $\begin{array}{l}\text { Several items on alcohol use, for } \\
\text { this report only drinking in past } \\
\text { month (any vs. none) and binge } \\
\text { drinking in past month. }\end{array}$ & $\begin{array}{l}\text { All effects ss if not marked nss } \\
\text { Any indicator: Drinking: } 1.5 \text {, heavy drinking } 1.4 \\
\text { Identity: drinking LG } 1.2 \text { nss, B } 1.2 \text { nss, HET/Q } \\
\text { with HO behav: } 1.8, Q \text { no HO behav: } 0.5 \text {, binge } \\
\text { drinking LG } 1.9, \mathrm{~B} 1.6, \mathrm{HET} / \mathrm{Q} \text { with } \mathrm{HO} \text { behav: } \\
\text { 1.7, Q no HO behav: } 0.5 \\
\text { Behavior: drinking: HO } 1.1 \mathrm{nss}, \mathrm{B} 1.6 \text {, binge } \\
\text { drinking: HO } 1.1 \mathrm{nss}, \mathrm{B} 1.6\end{array}$ & $\begin{array}{l}\text { For behavior only, larger } \\
\text { effects among } \mathrm{B} \text { than } \mathrm{HO}\end{array}$ & $\begin{array}{l}\text { Larger effect } \\
\text { among girls. }\end{array}$ & \\
\hline $\begin{array}{l}\text { Tucker, } \\
\text { Ellickson, \& } \\
\text { Klein (2008) }\end{array}$ & $\begin{array}{l}1,633 \text { girls, } 14 \text { years-old in first } \\
\text { wave, from } 30 \text { schools in } \\
\text { California and Oregon, } \\
\text { followed up at age } 18 \text { and } 23 .\end{array}$ & $\begin{array}{l}8.7 \% \text { classified as B based on } \\
\text { attraction in the analytical sample } \\
(0.8 \% \text { as } L \text { in the whole sample, } \\
\text { excluded from analysis). } \\
\text { Method? }\end{array}$ & $\begin{array}{l}\text { Frequency/ quantity of alcohol, } \\
\text { tobacco, marijuana. } \\
\text { Current use = ever in past } \\
\text { month. } \\
\text { Problematic use based on \# } \\
\text { events with problematic } \\
\text { outcome as result of } \\
\text { drug/alcohol use, e.g. missing } \\
\text { school, physical fights etc. } \\
\text { Mental Health with the MHI-5 } \\
\text { scale. }\end{array}$ & $\begin{array}{l}\text { Current cigarette use with } 14,18,23 \text { years, all } \\
\text { RRs all ss: } 1.5,1.5,1.8 \\
\text { Current alcohol: } 1.3 \mathrm{ss}, 1.1 \mathrm{nss}, 1.1 \mathrm{nss} \\
\text { Current marijuana: } 1.3,1.5 \mathrm{ss}, 2.2 \mathrm{ss} \\
\text { \# Cigarettes: } d=0.2,0.2,0.2 \text {, all nss } \\
\text { \# Alcohol: } d=0.1 \mathrm{nss}, 0.1 \mathrm{nss}, 0.3 \mathrm{ss} \\
\text { \# Marijuana: } d=0.1 \mathrm{nss}, 0.1 \mathrm{nss}, 0.2 \text {, ss } \\
\text { Problematic use: effect size n.a. but ss elevated at } \\
\text { age } 23 \\
\text { Good mental health }(14,18) \\
d=-0.2,-0.3 \text {, all ss }\end{array}$ & None reported & - & Only B girls \\
\hline
\end{tabular}


Online supplemental material to Plöderl \& Tremblay (2015). Mental health of sexual minorities. A systematic review. International Review of Psychiatry. http://dx.doi.org/10.3109/09540261.2015.1083949

\section{Add-Health Studies}

Nationally representative sample of adolescents in grades 7-12 in the United States during the 1994-95 school year. The Add Health cohort has been followed into young adulthood with four in-home interviews.

Wave I: 1994/1995, adolescents Grades 7-12, aged? , Wave II: 1996, adolescents, aged?, Wace III: 2001/2002, young adults, 18-26 years, Wave IV: 2007/2008, young adults aged 24-32,

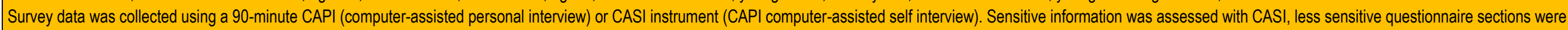
administered with the assistance of CAPI.

\begin{tabular}{|c|c|c|c|c|c|c|c|}
\hline Author (Year) & Sample & $\begin{array}{l}\text { Percentage of Sexual Minority } \\
\text { Individuals, Dimensions of } \\
\text { Sexual Orientation, } \\
\text { Assessment Method }\end{array}$ & Outcome Variables & $\begin{array}{l}\text { Main Results (SM vs. HET) } \\
\text { All Effects ORs if not other specified }\end{array}$ & Subgroup Differences & $\begin{array}{l}\text { Gender } \\
\text { Differences }\end{array}$ & Note \\
\hline $\begin{array}{l}\text { Almazan, } \\
\text { Roettger, \& } \\
\text { Acosta (2014) }\end{array}$ & $\begin{array}{l}14,625 \text { young adults, } \\
\text { Wave IV }\end{array}$ & $\begin{array}{l}9.5 \% \text { of women and } 4.1 \% \text { of men } \\
\text { reported some degree of } \mathrm{HO} \\
\text { attraction, } 19.9 \% \text { and } 6.4 \% \\
\text { nonheterosexual identity, } 14.2 \% \\
\text { and } 6.0 \% \text { lifetime } \mathrm{HO} \text { behavior, } \\
4.4 \% \text { and } 3.1 \% \text { past } 12 \text { month } \mathrm{HO} \\
\text { sexual behavior }\end{array}$ & $\begin{array}{l}\text { SA in past } 12 \text { months, single } \\
\text { item. }\end{array}$ & $\begin{array}{l}\text { Women } \\
\text { attraction } 2.0 \text { nss. Identity } 2.1 \mathrm{~s} \text {, } \\
\text { behavior: lifetime } 2.1 \mathrm{ss} \text {, past year } 2.2 \mathrm{ss} \text {. } \\
\text { Men, all nss } \\
\text { attraction } 0.2 \text {, identity } 0.6 \text {, } \\
\text { behavior: lifteime } 0.6 \text {, past year } 0.7\end{array}$ & None reported & $\begin{array}{l}\text { Small SO effect } \\
\text { among women } \\
\text { but none or } \\
\text { reversed effect } \\
\text { among men. }\end{array}$ & $\begin{array}{l}\text { Later Coming } \\
\text { Out process } \\
\text { among women } \\
\text { responsible? }\end{array}$ \\
\hline $\begin{array}{l}\text { Cardom, } \\
\text { Rostosky, \& } \\
\text { Danner (2013) } \\
\text { b }\end{array}$ & $\begin{array}{l}\text { Wave I and IV. } \\
\text { Subset of 1,591 depressed } \\
\text { adolescents/ young adults } \\
\text { (above cutoff in CES-D) at } \\
\text { Wave I who also were } \\
\text { assessed for SO at Wave IV }\end{array}$ & $\begin{array}{l}20 \% \text { identified as SM at Wave IV } \\
(14 \% \text { moHET, } 3 \% \text { B, } 1 \% \text { moG }, 1 \% \\
\text { G). }\end{array}$ & $\begin{array}{l}\text { Depression (past week) with } \\
\text { CES-D scale at both waves, SA } \\
\text { in past year }\end{array}$ & $\begin{array}{l}\text { Depression (all effects Cohen's d) } \\
\text { Wave I: moHET 0.3 ss, B } 0.3 \text { nss, } \\
\text { moHO } 0.3 \text { nss, HO 0.1 nss, } \\
\text { Wave IV: moHET } 0.4 \text { ss, B } 0.5 \text { ss, } \\
\text { moHO 0.2 nss, HO 0.1 nss } \\
\text { SA (all effects RRs) } \\
\text { Wave I: moHET 2.3, B 2.0, moHO 1.9, HO } 0.5 \\
\text { Wave IV: moHET: 1.3, B 5.8, moG n.a., G } 1.5 .\end{array}$ & $\begin{array}{l}\text { Depression, only ss effects } \\
\text { for moHET (Wave I and IV) } \\
\text { and B in Wave IV. } \\
\text { SA: effects in Wave I for all } \\
\text { subgroups except G youth } \\
\text { (reversed effect). In Wave } \\
\text { IV, effects only pronounced } \\
\text { for GB youth. }\end{array}$ & None reported & $\begin{array}{l}\text { SO variable in } \\
\text { Wave IV was } \\
\text { used also for } \\
\text { Wave I } \\
\text { analysis. }\end{array}$ \\
\hline $\begin{array}{l}\text { Everett \& } \\
\text { Mollborn } \\
(2013)\end{array}$ & Wave IV & $\begin{array}{l}+++ \\
\mathrm{B} \text { and moHET were combined } \\
\text { (4.1\% men and } 17.8 \% \text { of women) } \\
\text { as well as } \mathrm{moHO} \text { and } \mathrm{HO}(2.7 \% \text { of } \\
\text { men and } 1.8 \% \text { of women). }\end{array}$ & $\begin{array}{l}\text { Past year binge drinking ( } \geq 2 \\
\text { times a month), past year drug } \\
\text { use, current smoking }\end{array}$ & $\begin{array}{l}\text { Men: Smoking } 0.9 \text { for } B \text { and } G \text {; for binge drinking } \\
\text { and drug use } 1.2-2.0 \text { and ss except binge drinking } \\
\text { for } G \text { men. } \\
\text { Women: all effects ss, } 1.6-4.9\end{array}$ & $\begin{array}{l}\text { Comparable for men, but } \\
\text { for women, } L \text { had larger } \\
\text { effects than } B \text { for binge } \\
\text { drinking and drug use }\end{array}$ & $\begin{array}{l}\text { Clearly larger } \\
\text { effects among } \\
\text { women }\end{array}$ & $\begin{array}{l}\text { MoHET were } \\
\text { grouped with B }\end{array}$ \\
\hline $\begin{array}{l}\text { Fried, Williams, } \\
\text { Cabral, \& } \\
\text { Hacker }(2013)^{b}\end{array}$ & $\begin{array}{l}\text { Wave II }(n=1,648) \text { and III }(n= \\
1,728)\end{array}$ & $\begin{array}{l}\text { HO attraction, not further specified, } \\
\text { no percentages given }\end{array}$ & SA in past year & $\begin{array}{l}\text { Wave II } 3.4 \text { ss } \\
\text { Wave III } 2.0 \text { nss }\end{array}$ & None reported & None reported & \\
\hline $\begin{array}{l}\text { Hahm, Wong, } \\
\text { Huang, } \\
\text { Ozonoff, \& Lee } \\
(2008)^{\text {b }}\end{array}$ & $\begin{array}{l}\text { 1,169 Asian Americans and } \\
\text { Pacific Islanders from Wave II } \\
\text { and III }\end{array}$ & $\begin{array}{l}\text { 8.7\% identified as SM in Wave III } \\
\text { (moHET, B, predHO, or HO), } \\
\text { asexual and Q were excluded }\end{array}$ & $\begin{array}{l}\text { Regular smoking in past year, } \\
\text { binge drinking (at least once in } \\
\text { past year), past year marijuana, } \\
\text { past year other illicit drugs }\end{array}$ & $\begin{array}{l}\text { Wave II: males (RRs } 0.6-1.1 \text { all nss), and } \\
\text { reversed effects among females (RRs } 0.5-0.8 \text {, ss } \\
\text { only for binge drinking. } \\
\text { Wave III: males (robacco: RR 0.8, nss, binge } \\
\text { drinking RR } 1.0 \text {, nss, marijuana RR } 2.3 \text { ss and } \\
\text { other drug use RR } 3.1 \mathrm{ss} \text { ). }\end{array}$ & None reported & $\begin{array}{l}\text { Reversed effect } \\
\text { for females in } \\
\text { Wave II }\end{array}$ & $\begin{array}{l}\text { Emerging SO } \\
\text { effect for } \\
\text { marijuana and } \\
\text { other illicit drug } \\
\text { use from Wave } \\
\text { II to Wave III }\end{array}$ \\
\hline
\end{tabular}


Online supplemental material to Plöderl \& Tremblay (2015). Mental health of sexual minorities. A systematic review. International Review of Psychiatry. http://dx.doi.org/10.3109/09540261.2015.1083949

\begin{tabular}{|c|c|c|c|c|c|c|c|}
\hline & & & & Females: 1.5, 1.3, 2.4, 6.0, all ss. & & & \\
\hline $\begin{array}{l}\text { Lindley, } \\
\text { Walsemann, \& } \\
\text { Carter (2012) }\end{array}$ & $\begin{array}{l}\text { Wave IV } \\
14,412 \text { young adults }\end{array}$ & +++ & Depression with CES-D & $\begin{array}{l}\text { Women: } \\
\text { Identity: ss for moHET, B, moHO but not } \mathrm{HO} \text {. } \\
\text { Attraction: ss effects for B but not } \mathrm{HO} \text {. } \\
\text { Behavior: ss for moHET but not } \mathrm{B}, \mathrm{moHO} \text {, or } \mathrm{HO} \\
\text { Men: } \\
\text { Only ss effects for moHET (identity and } \\
\text { behavior).Effect sizes n.a. }\end{array}$ & $\begin{array}{l}\text { Only ss effects for moHET } \\
\text { men/women and for } \\
\text { moHET men. } \\
\text { B men and women } \\
\text { comparable }\end{array}$ & $\begin{array}{l}\text { Somewhat } \\
\text { smaller effects } \\
\text { among men }\end{array}$ & \\
\hline $\begin{array}{l}\text { Loosier \& } \\
\text { Dittus (2010) }\end{array}$ & $\begin{array}{l}\text { Wave III } \\
14,322 \text { young adults }\end{array}$ & $\begin{array}{l}5.7 \% \text { of men and } 13.9 \% \text { of women } \\
\text { identified as SM in Wave III. } \\
\% \text { of same sex romantic partners: } \\
\text { HET } 0 \% \text {, moHET } 2 \% \text {, B } 15 \% \text {, } \\
\text { moHO } 68 \%, \text { HO } 90 \% \text { across } \\
\text { Waves I-III }\end{array}$ & $\begin{array}{l}\text { Depression (CES-D), } \\
\text { \# of past year drinking, }\end{array}$ & $\begin{array}{l}\text { Depression and alcohol use was elevated among } \\
\text { all SO subgroups (effect size n.a.) but only ss (in } \\
\text { planned contrast analysis) for B and moHET. }\end{array}$ & $\begin{array}{l}\text { Larger effects for } B \text { and } \\
\text { moHET }\end{array}$ & None reported & \\
\hline $\begin{array}{l}\text { Marshal, } \\
\text { Dermody, } \\
\text { Cheong, et al. } \\
\text { (2013) }\end{array}$ & $\begin{array}{l}12379 \text { adolescents / young } \\
\text { adults, Waves I - IV } \\
\text { Selection based on age for } \\
\text { homogenity (Wave I: } 14-18 \text {, } \\
\text { Wave II: } 15-19 \text {, Wave III: } 20- \\
\text { 24, Wave IV: } 27-31 \text { ) }\end{array}$ & $\begin{array}{l}\text { Wave IV information for SO: } \\
13.2 \text { identified as SM in Wave IV } \\
(2.1 \% \text { LG or moLG, } 1.5 \% \text { B, } 9.6 \% \\
\text { moHET) }\end{array}$ & Depression with CES-D & $\begin{array}{l}\text { In latent growth analysis using structural equation } \\
\text { modeling, all SM groups differed significantly from } \\
\text { HET and the increase was comparable through all } \\
\text { waves. }\end{array}$ & $\begin{array}{l}\text { B had ss larger levels than } \\
\text { moHET and moHET hat ss } \\
\text { larger levels than } \\
\text { LG/moLG. }\end{array}$ & Not reported & $\begin{array}{l}\text { Ss effects are } \\
\text { in contrast to } \\
\text { other studies. }\end{array}$ \\
\hline $\begin{array}{l}\text { Marshal, King, } \\
\text { et al. (2012) }\end{array}$ & $\begin{array}{l}7,765 \text { girls/women, } \\
\text { Waves I - IV, } \\
\text { selection based on age at } \\
\text { Wave I (12-18 years) }\end{array}$ & $\begin{array}{l}+++ \\
\text { Wave IV information for SO: } \\
19.6 \% \text { identified as SM in Wave IV } \\
(4.2 \% \text { L, moL, or B, and } 15.5 \% \\
\text { moHET) }\end{array}$ & $\begin{array}{l}\text { Past month smoking (\# days); } \\
\text { past year alcohol use and binge } \\
\text { drinking, \# drunkenness in past } \\
\text { year (likert 0-7, never to almost } \\
\text { every day) }\end{array}$ & $\begin{array}{l}\text { Ss effects for both LB and moHET girls in Wave I; } \\
\text { increasing difference from Wave I to Wave IV into } \\
\text { young adulthood. }\end{array}$ & $\begin{array}{l}\text { LG and B comparable (not } \\
\text { ss different) }\end{array}$ & - & \\
\hline $\begin{array}{l}\text { McLaughlin, } \\
\text { Hatzenbuehler, } \\
\text { Xuan, \& } \\
\text { Conrond } \\
(2012)^{\text {b }}\end{array}$ & $\begin{array}{l}13962 \text { young adults, } \\
\text { Wave III }\end{array}$ & $\begin{array}{l}3.4 \% \text { identified as } L G B \text { ( } 48 \% \text { of } \\
\text { them as B). moHET were excluded } \\
\text { from analysis, } L G \text { and moLG were } \\
\text { combined. }\end{array}$ & $\begin{array}{l}\text { Depression with the CED-D, } \\
\text { past month smoking, illicit drug } \\
\text { use since Wave I, binge drinking } \\
\text { in past year, consequences of } \\
\text { drinking and drug abuse. }\end{array}$ & $\begin{array}{l}\text { For all measures there were elevated levels } \\
\text { among SM (ss?, effect size n.a.). Binge drinking } \\
\text { (RRs): LG 1.4, B 1.2, illicit drug use: LG 2.5, B } 2.4\end{array}$ & $\begin{array}{l}\text { LG and B comparable for } \\
\text { binge drinking and drug use } \\
\text { but B somewhat elevated } \\
\text { for depression and tobacco } \\
\text { use. }\end{array}$ & Not reported & \\
\hline $\begin{array}{l}\text { Needham } \\
\text { (2012) }\end{array}$ & $\begin{array}{l}8,151 \text { adolescents/ young } \\
\text { adults, } \\
\text { Waves I - IV }\end{array}$ & $\begin{array}{l}\text { Sexual attraction across the } 4 \\
\text { waves, classified in consistent SM } \\
(1.3 \%) \text {, transition to SM }(6.0) \text {, } \\
\text { transition to HET }(5 \%) \text {, consistent } \\
\text { HET }(87.6 \%) \text {. }\end{array}$ & $\begin{array}{l}\text { Depression (CES-D), past } \\
\text { month frequency smoking, past } \\
\text { year heavy drinking, past month } \\
\text { marijuana }\end{array}$ & $\begin{array}{l}\text { Effects from unconditional growth models, } \\
\text { consistent HET as reference group. No ss effects } \\
\text { for the rate of change over time for any subgroup. } \\
\text { Females: consistent SM and those who transit to } \\
\text { SM start with ss elevated depression and nearly } \\
\text { all substance abuse variables. Those who transit } \\
\text { to HET initially do not have ss increased }\end{array}$ & $\begin{array}{l}\text { Comparable trajectories } \\
\text { over time. }\end{array}$ & $\begin{array}{l}\text { Larger effects } \\
\text { among females } \\
\text { than males. }\end{array}$ & \\
\hline
\end{tabular}




\begin{tabular}{|c|c|c|c|c|c|c|c|}
\hline & & & & $\begin{array}{l}\text { depression but elevated levels of smoking, } \\
\text { drinking, and marijuana. } \\
\text { Males: consistent SM start with ss increased } \\
\text { depression but with nss different substance abuse } \\
\text { levels. Nss different levels of depression for those } \\
\text { who transit to SM and ss lower levels of smoking } \\
\text { and nss differences for other substance abuse. } \\
\text { Those who transit to HET have initially ss } \\
\text { increased depression and substance abuse. }\end{array}$ & & & \\
\hline $\begin{array}{l}\text { Needham \& } \\
\text { Austin (2010) }\end{array}$ & $\begin{array}{l}11,153 \text { young adults, } \\
\text { Wave III } \\
\text { Those with no contact to } \\
\text { parents and those married } \\
\text { excluded }\end{array}$ & $\begin{array}{l}+++ \\
\text { Identified as SM (LG or moLG, B, } \\
\text { moHET or HET) }\end{array}$ & $\begin{array}{l}\text { High depression (CES-D } \geq 10 \\
\text { for men and } \geq 11 \text { for women), } \\
\text { binge drinking, past year binge } \\
\text { drinking } 1-2 \text { times per week, any } \\
\text { past month marijuana or hard } \\
\text { drug use. }\end{array}$ & $\begin{array}{l}\text { Women: } \\
\text { All effects RRs and ss: depression: L 1.4 , B 1.8; } \\
\text { binge drinking: L 1.5, B 1.9, marijuana: L 2.6, B } \\
\text { 2.4; hard drugs: L 4.2, B 3.9 } \\
\text { Men: } \\
\text { all effects nss: depression: G 1.7, B 2.0; binge } \\
\text { drinking: G and B 0.7, marijuana: G 0.9, B 1.4; } \\
\text { hard drugs G 1.3, B 1.7 }\end{array}$ & $\begin{array}{l}L \text { and } B \text { women } \\
\text { comparable; } \\
B \text { men somewhat elevated } \\
\text { than } G \text { men. }\end{array}$ & $\begin{array}{l}\text { Comparable for } \\
\text { depression, larger } \\
\text { effects among } \\
\text { women for } \\
\text { marijuana and } \\
\text { hard drugs. }\end{array}$ & \\
\hline $\begin{array}{l}\text { Pearson \& } \\
\text { Wilkinson } \\
(2013)^{b}\end{array}$ & $\begin{array}{l}\text { 13,140 adolescents, } \\
\text { Wave I and II }\end{array}$ & $\begin{array}{l}4.1 \% \text { of girls and } 6.9 \% \text { of boys } \\
\text { reported lifetime attraction towards } \\
\text { same-sex persons in Wave I }\end{array}$ & $\begin{array}{l}\text { Binge drinking in Wave II, } \\
\text { illegal drug use, both since } \\
\text { Wave I. }\end{array}$ & $\begin{array}{l}\text { Depression: girls } d=0.3 \text {, boys } d=0.4 \text {, all ss. } \\
\text { Binge drinking: girls } d=0.2 \mathrm{ss} \text {, boys } d=0.0 \text {, nss. } \\
\text { llicit drug use: girls } d=0.3 \text {, boys } d=0.1 \text {, all ss. }\end{array}$ & None reported & $\begin{array}{l}\text { Larger effects for } \\
\text { girls for alcohol/ } \\
\text { drugs but not } \\
\text { depression }\end{array}$ & Measure of SO \\
\hline $\begin{array}{l}\text { Russell \& } \\
\text { Joyner (2001) }\end{array}$ & $\begin{array}{l}11,940 \text { adolescents, } \\
\text { Wave I }\end{array}$ & $\begin{array}{l}8.0 \% \text { of boys and } 6.6 \% \text { of girls } \\
\text { either ever had a same-sex } \\
\text { romantic attraction or same-sex } \\
\text { romantic relationship. }\end{array}$ & $\begin{array}{l}\text { Past week depression with CES- } \\
\text { D scale, 9-item scale on past } \\
\text { year alcohol abuse, single items } \\
\text { on SA }\end{array}$ & $\begin{array}{l}\text { Ss elevated levels of depression and alcohol } \\
\text { abuse (effect size n.a.). } \\
\text { SA: } 2.5\end{array}$ & None reported & $\begin{array}{l}\text { Comparable for } \\
\text { SA }\end{array}$ & \\
\hline $\begin{array}{l}\text { Savin-Williams, } \\
\text { Cohen, Joyner, } \\
\text { \& Rieger } \\
(2010)^{b}\end{array}$ & $\begin{array}{l}\text { 6,615 young adult men, } \\
\text { Wave III }\end{array}$ & $\begin{array}{l}+++ \\
3.2 \% \text { identified (?) as moHET, } \\
0.6 \% \text { as B, } 1.8 \text { as HO. }\end{array}$ & Depression with CES-D & $\begin{array}{l}\text { Ss effects for moHET (ss, BF 12.8, strong } \\
\text { evidence, } d=4.4 \text { ), B (nss, BF 1.3, weak } \\
\text { evidence, } d=2.5 \text { ) HO (ss, BF 3.3, subst. } \\
\text { evidence, } d=3.8 \text { ). }\end{array}$ & $\begin{array}{l}\text { B somewhat elevated } \\
\text { compared to } \mathrm{HO} \text {. }\end{array}$ & - & $\begin{array}{l}\text { d's may be } \\
\text { wrong due to } \\
\text { likely wrong SD } \\
(0.07) \text { of HET } \\
\text { males in paper }\end{array}$ \\
\hline $\begin{array}{l}\text { Silenzio, Pena, } \\
\text { Duberstein, } \\
\text { Cerel, \& Knox } \\
(2007)^{b}\end{array}$ & $\begin{array}{l}\text { 14,322 young adults, } \\
\text { Wave III }\end{array}$ & $\begin{array}{l}\text { SM based on self-identification } \\
\text { (based on attraction) as } \mathrm{HO} \text {, } \\
\text { moHO or B. MoHET and not } \\
\text { attracted put in reference group }\end{array}$ & $S A$ in past year & Ss effect, 3.0. & None reported & None reported & $\begin{array}{l}\text { Reference } \\
\text { group includes } \\
\text { moHET and } \\
\text { not attracted. }\end{array}$ \\
\hline $\begin{array}{l}\text { Strutz, Herring, } \\
\text { \& Halpern } \\
\text { (2015) }\end{array}$ & $\begin{array}{l}\text { 13,088 young adults, } \\
\text { Wave IV }\end{array}$ & $\begin{array}{l}21.4 \% \text { had at least one indicator of } \\
\text { SM status and } 5.6 \% \text { all three } \\
\text { indicators. } 3.7 \% \text { had an attraction } \\
\text { indicator plus LGB identity or }\end{array}$ & $\begin{array}{l}\text { Lifetime diagnosis of } \\
\text { depression, anxiety/PD, ADHD, } \\
\text { and depression on the CES-D } \\
\text { (above cutoff). }\end{array}$ & $\begin{array}{l}\text { All effects crude ORs, mostly ss. } \\
\quad \text { Women (all effects ss) } \\
\text { Any indicator: depression 2.5, anxiety/PD 2.2, } \\
\text { ADHD 1.9, depression 2.2. }\end{array}$ & $\begin{array}{l}\text { Larger effects when SM } \\
\text { status on all three } \\
\text { indicators. }\end{array}$ & $\begin{array}{l}\text { Somewhat larger } \\
\text { effects among } \\
\text { women. }\end{array}$ & \\
\hline
\end{tabular}


Online supplemental material to Plöderl \& Tremblay (2015). Mental health of sexual minorities. A systematic review. International Review of Psychiatry. http://dx.doi.org/10.3109/09540261.2015.1083949

\begin{tabular}{|c|c|c|c|c|c|c|c|}
\hline & & $\begin{array}{l}\text { behavioral indicator. } \\
\text { Based on number of indicators of } \\
\text { SO in Wave IV (any current HO } \\
\text { attraction, any lifetime same-sex } \\
\text { romantic or sexual partners), } \\
\text { identity (any nonheterosexual } \\
\text { including moHET). }\end{array}$ & & $\begin{array}{l}\text { All indicators: depression } 3.4 \text {, anxiety/PD 2.8, } \\
\text { ADHD 2.7, depression } 2.7 \\
\text { Attraction plus other indicators: depression } 2.7, \\
\text { anxiety/PD 2.3, ADHD } 2.8 \text {, depression } 2.5 \\
\quad \text { Men } \\
\text { Any indicator: depression } 2.5 \mathrm{ss} \text {, anxiety/PD } 1.6 \\
\text { ss, ADHD } 1.4 \text { nss, depression } 1.5 \mathrm{ss} \text {. } \\
\text { All indicators: depression } 3.6 \mathrm{ss} \text {, anxiety/PD } 2.9 \mathrm{ss}, \\
\text { ADHD } 1.7 \text { nss, depression } 1.3 \text { nss. } \\
\text { Attraction plus other indicators: depression } 3.5 \mathrm{ss} \text {, } \\
\text { anxiety/PD } 2.6 \mathrm{ss}, \text { ADHD } 1.1 \mathrm{nss} \text {, depress.1.2 nss }\end{array}$ & & & \\
\hline $\begin{array}{l}\text { Teasdale \& } \\
\text { Bradley-Engen } \\
(2010)\end{array}$ & $\begin{array}{l}\text { 11,911 adolescents, } \\
\text { Wave II }\end{array}$ & $\begin{array}{l}7 \% \text { classifieds as SM based on } \\
\text { lifetime romantic attraction to } \\
\text { person of same sex. }\end{array}$ & Depression (CES-D) & Ss effect for depression, effect size n.a. & None reported & Not reported & \\
\hline Ueno (2010b) & Wave I-III & $\begin{array}{l}4 \text { categories based on all } 3 \\
\text { indicators of SM (identity, } \\
\text { behavior, attraction): HET (no SM } \\
\text { indicator = baseline), HOadol (any } \\
\text { indicator only in adolescence, } \\
6.4 \% \text { women, } 8.8 \% \text { men), } \\
\text { HOyadul (any indicator only in } \\
\text { young adulthood } 12.7 \% \text { and } \\
6.2 \% \text { ), HOboth (any indicator in } \\
\text { both life-stages } 4.1 \% \text { and } 2.0 \% \text { ) }\end{array}$ & $\begin{array}{l}\text { Depression (CES-D), binge } \\
\text { drinking in past year and drug } \\
\text { use in last month }\end{array}$ & $\begin{array}{l}\text { SM only in adolescence: greater levels of mental } \\
\text { health problems in adolescence but no worse } \\
\text { mental health changes into young adulthood. } \\
\text { SM only in young adulthood: show worse } \\
\text { changes. } \\
\text { Among females: SM in both life stages associated } \\
\text { with worse mental health changes. } \\
\text { However, these differences are modest, and } \\
\text { substantial amounts of variations in mental health } \\
\text { changes are observed within each group. }\end{array}$ & & $\begin{array}{l}\text { Larger effects } \\
\text { among females }\end{array}$ & \\
\hline $\begin{array}{l}\text { Williams \& } \\
\text { Chapman } \\
(2011)^{b}\end{array}$ & $\begin{array}{l}18,924 \text { adolescents, } \\
\text { Wave I }\end{array}$ & $\begin{array}{l}7.5 \% \text { SM (if ever having either/or } \\
\text { romantic HO attraction, romantic } \\
\text { HO relationship, same-sex sexual } \\
\text { partner, all in past } 18 \text { months) }\end{array}$ & $\begin{array}{l}\text { Depression (CES-D scale } \geq 20 \text { ), } \\
\text { anxiety (6-item scale, cutoff } \geq> \\
\text { 18), SA }\end{array}$ & $\begin{array}{l}\text { Ss effects for anxiety } 2.0 \text {, depression } 1.8 \text {, } \\
\text { SA } 2.4\end{array}$ & None reported & None reported & \\
\hline
\end{tabular}




\section{Growing UP Today Study (GUTS)}

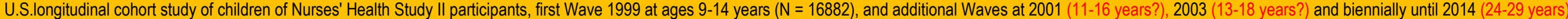

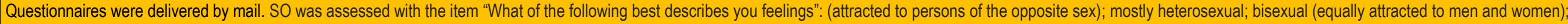
mostly homosexual; completely homosexual (gay/lesbian, attracted to persons of the same sex); and not sure.

In 2007, there was also an item on sexual behavior "During your life, the persons with whom you have had sexual contact are?" No sexual contact, females, males, or both

\begin{tabular}{|c|c|c|c|c|c|c|c|}
\hline Author (Year) & Sample & $\begin{array}{l}\text { Percentage of Sexual Minority } \\
\text { Individuals, Dimensions of } \\
\text { Sexual Orientation, } \\
\text { Assessment Method }\end{array}$ & Outcome Variables & $\begin{array}{l}\text { Main Results (SM vs. HET) } \\
\text { All Effects ORs if not other specified }\end{array}$ & Subgroup Differences & \begin{tabular}{|l} 
Gender \\
Differences
\end{tabular} & Note \\
\hline $\begin{array}{l}\text { Corliss, } \\
\text { Rosario, Wypij, } \\
\text { Fisher, \& } \\
\text { Austin (2008) }\end{array}$ & $\begin{array}{l}13,450 \text { adolescents from } \\
\text { waves I, II, III who responded } \\
\text { to SO and drug use items in at } \\
\text { least one wave. Multivariable } \\
\text { generalized estimating } \\
\text { equations (GEE) repeated } \\
\text { measures modified Poisson } \\
\text { regression was used to adjust } \\
\text { for dependency of data across } \\
\text { waves }\end{array}$ & $\begin{array}{l}+++ \\
8.5 \% \text { of boys and } 16.1 \% \text { of girls } \\
\text { reported a SM orientation. } \\
\text { moHO and } \mathrm{HO} \text { were combined } \\
\text { into LG category, } \mathrm{Q} \text { were } \\
\text { excluded. }\end{array}$ & $\begin{array}{l}\text { Any past month drinking and \# } \\
\text { of binge drinking episodes; i.e. } \geq \\
5 \text { drinks (males) or } \geq 4 \text { drinks in } \\
\text { a row (females) in past year. }\end{array}$ & $\begin{array}{l}\text { Any past month drinking, RR } \\
\text { Males: moHET } 1.2 \mathrm{ss}, \mathrm{B} 1.5 \mathrm{ss}, \mathrm{G} 1.2 \mathrm{nss} \\
\text { Females: } 1.4 \mathrm{ss}, 1.6 \mathrm{ss}, 1.1 \mathrm{nss} \\
\text { moHE males and females and B females reported } \\
\text { greater alcohol use on all } 3 \text { outcomes. } \\
\text { B males reported greater alcohol use but } \\
\text { associations were ss only for any past-month } \\
\text { drinking. } \\
\text { G reported consuming a larger number of drinks. } \\
\text { Compared with HET females, } L \text { reported a greater } \\
\text { number of drinks usually consumed and binge } \\
\text { drinking episodes but the latter was not ss. }\end{array}$ & & $\begin{array}{l}\text { For \#drinks ss } \\
\text { larger SO } \\
\text { difference for B } \\
\text { females } \\
\text { compared to } \\
\text { males and for } \\
\text { binge drinking } \\
\text { larger SO effect } \\
\text { among moHET } \\
\text { females than } \\
\text { males }\end{array}$ & $\begin{array}{l}\text { The RR were } \\
\text { adjusted for } \\
\text { household } \\
\text { adult alcohol } \\
\text { drinking }\end{array}$ \\
\hline $\begin{array}{l}\text { Corliss et al. } \\
(2010)^{b}\end{array}$ & $\begin{array}{l}12,664 \text { adolescents from } \\
\text { waves I, II, III who responded } \\
\text { to SO and drug use items in at } \\
\text { least one wave. } \\
\text { Statistical modeling as in } \\
\text { Corliss, et al. (2008) }\end{array}$ & $\begin{array}{l}+++ \\
\text { moHO and } \mathrm{HO} \text { were combined } \\
\text { into } \mathrm{LG} \text { category, } \mathrm{Q} \text { were } \\
\text { excluded. }\end{array}$ & $\begin{array}{l}\text { Past year use of different drugs } \\
\text { (marijuana, ecstasy, cocaine, } \\
\text { heroin, amphetamine, LSD) and } \\
\text { medication not prescribed by a } \\
\text { doctor. Results only given for } \\
\text { marijuany and for any other illicit } \\
\text { drug use (because results are } \\
\text { comparable) }\end{array}$ & $\begin{array}{l}\text { All effects RRs and ss. } \\
\quad \text { Males (MoHET, B, G): } \\
\begin{array}{l}\text { Marijuana: } 1.9,1.7,1.6, \\
\text { Any other illicit drug: } 2.3,2.9,1.9 \\
\quad \text { Females (MoHET, B, L) } \\
\text { Marijuana: } 2.0,2.6,1.7 \\
\text { Any other illicit drug: } 3.4,5.1,3.1 \\
\text { [For all drugs and medication, RRs ranged from } \\
\text { 1.3-9.4, nearly all were ss. Those } 12-17 \text { years old } \\
\text { had larger effects differences than those } 18-23 \mathrm{y} .]\end{array}\end{array}$ & $\begin{array}{l}\text { For girls, B had larger SO } \\
\text { differences than L or } \\
\text { moHET, (which were } \\
\text { comparable). } \\
\text { For boys, SO differences of } \\
\text { B were comparable to } G \\
\text { and moHET except for } \\
\text { ecstasy and heroin where B } \\
\text { had larger SO differences }\end{array}$ & $\begin{array}{l}\text { Overall, SO } \\
\text { differences were } \\
\text { larger among girls } \\
\text { than boys. }\end{array}$ & $\begin{array}{l}\text { Younger SM } \\
\text { were more at } \\
\text { risk }\end{array}$ \\
\hline $\begin{array}{l}\text { Roberts, } \\
\text { Rosario, } \\
\text { Slopen, Calzo, } \\
\text { \& Austin (2013) }\end{array}$ & $\begin{array}{l}10,566 \text { adolescents/ young } \\
\text { adults, } 11-30 \text { years old, } 1999- \\
2010 \text { waves, subset of } \\
\text { responders to gender } \\
\text { nonconformity items in } 2005 \\
\text { or } 2007 \text {. }\end{array}$ & $\begin{array}{l}\text { ? \% were classified as SM } \\
\text { according to identity (LG, B, } \\
\text { moHET), and also HET identified } \\
\text { with homosexual experiences } \\
\text { (HET-HO), Based on } 2007 \\
\text { measure, Q excluded. }\end{array}$ & Depression with CES-D scale & $\begin{array}{l}\text { Results only available from multivariate model, } \\
\text { adjusted for childhood gender nonconformity, but } \\
\text { ss effects among HET-HO, moHET, B, LG }\end{array}$ & Largest effect among B & None reported & $\begin{array}{l}\text { Biased effects } \\
\text { due to } \\
\text { adjustment }\end{array}$ \\
\hline $\begin{array}{l}\text { Rosario, } \\
\text { Reisner, }\end{array}$ & $\begin{array}{l}\text { 5,193 adolescents/ young } \\
\text { adults, waves, 2003, 2005, }\end{array}$ & $\begin{array}{l}\text { ?\% were classified as SM } \\
\text { according to identity (LG and }\end{array}$ & $\begin{array}{l}\text { Past year substance use for this } \\
\text { paper: smoking (any and > }\end{array}$ & $\begin{array}{l}\text { Effects all ss if note described as nss } \\
\text { Smoking: } L G ~ 2.6, B 2.8, \text { moHET } 2.3\end{array}$ & $\begin{array}{l}\text { Comparable effects among } \\
\text { subgroups }\end{array}$ & & \\
\hline
\end{tabular}


Online supplemental material to Plöderl \& Tremblay (2015). Mental health of sexual minorities. A systematic review. International Review of Psychiatry. http://dx.doi.org/10.3109/09540261.2015.1083949

\begin{tabular}{|c|c|c|c|c|c|c|}
\hline $\begin{array}{l}\text { Corliss, Wypij, } \\
\text { Calzo, et al. } \\
(2014)\end{array}$ & $\begin{array}{l}2007 \text { and with mothers reports } \\
\text { from } 2004 \text { and } 2006\end{array}$ & $\begin{array}{l}\text { mostly LG combined, B, moHET), } \\
\text { Q excluded. }\end{array}$ & $\begin{array}{l}\text { weekly), marijuana (any and } \geq \\
\text { monthly), misuse prescription } \\
\text { drugs, any illicit drug use, binge } \\
\text { drinking ( } \geq 4 \text { drinks girls, } \geq 5 \\
\text { drinks boys, } \geq 3 \text { times past } \\
\text { year). Depressive Symptoms } \\
\text { subscale of the McKnight Risk } \\
\text { Factor Survey }\end{array}$ & $\begin{array}{l}\text { Binge drinking: } 1.3 \mathrm{nss}, 1.2 \mathrm{nss}, 1.6 \\
\text { Marijuana: } 2.5,3.4,27 \\
\text { Other illicit drugs: } 3.4,3.6,3.0 \\
\text { Prescription drug: } 1.6,2.2,2.0 \\
\text { Depression, all, ss (all d's): } 0.3,0.5,0.4\end{array}$ & & \\
\hline $\begin{array}{l}\text { Rosario, } \\
\text { Reisner, } \\
\text { Corliss, Wypij, } \\
\text { Frazier, et al. } \\
\text { (2014) b }\end{array}$ & $\begin{array}{l}\text { 6,122 adolescents/ young } \\
\text { adults from wave 2005, plus } \\
\text { those with reports from mother } \\
2004 \text { and } 2006 \text {. }\end{array}$ & $\begin{array}{l}13 \% \text { were classified as SM base } \\
\text { on identification ( } 1.7 \% \text { identified as } \\
\text { LG, } 1.7 \% \text { as } B, 10 \% \text { as moHET) }\end{array}$ & $\begin{array}{l}\text { Depression with the CES-D } \\
\text { scale }\end{array}$ & $\begin{array}{l}\text { Ss difference among all subgroups (d's) } \\
\text { LG } 0.2, B \text {, } 0.5 \text {, moHET } 0.4\end{array}$ & $\begin{array}{l}\text { Larger effect among } B \text { and } \\
\text { moHET }\end{array}$ & None reported \\
\hline $\begin{array}{l}\text { Ziyadeh et al. } \\
(2007)^{b}\end{array}$ & $\begin{array}{l}9731 \text { adolescents from } \\
\text { Wave I (1999) }\end{array}$ & $\begin{array}{l}\text { Identification based on description } \\
\text { of feelings, GB combined }\end{array}$ & $\begin{array}{l}\text { Only past year binge drinking for } \\
\text { this review. } \\
\text { Depressive Symptoms subscale } \\
\text { of the McKnight Risk Factor } \\
\text { Survey }\end{array}$ & $\begin{array}{l}\text { Binge drinking (all RRs.) } \\
\text { Boys: moHET } 1.5 \text { ss, GB } 1.3 \text { nss, Q } 0.7 n s s \\
\text { Girls: moHET } 1.9 \text { ss, LB } 2.3 \text { ss, Q } 0.4 \text { nss } \\
\text { Depression (effect size n.a.) } \\
\text { Males: ss for moHET and Q but not GB } \\
\text { Females: ss for all subgroups }\end{array}$ & $\begin{array}{l}\text { For girls, somewhat larger } \\
\text { effect among LB than } \\
\text { moHET, reversed effect for } \\
\text { Q. } \\
\text { For boys, no clear } \\
\text { subgroup difference. }\end{array}$ & $\begin{array}{l}\text { Larger effect } \\
\text { among LB girls } \\
\text { than BG boys, } \\
\text { no clear } \\
\text { difference for } \\
\text { other subgroups }\end{array}$ \\
\hline
\end{tabular}


Online supplemental material to Plöderl \& Tremblay (2015). Mental health of sexual minorities. A systematic review. International Review of Psychiatry. http://dx.doi.org/10.3109/09540261.2015.1083949

Other Adolescent Samples

\begin{tabular}{|c|c|c|c|c|c|c|c|}
\hline Author (Year) & $\begin{array}{l}\text { Sample, } \\
\text { Sampling Method }\end{array}$ & $\begin{array}{l}\text { Percentage of Sexual Minority } \\
\text { Individuals, Dimensions of } \\
\text { Sexual Orientation, } \\
\text { Assessment Method }\end{array}$ & Outcome Variables & $\begin{array}{l}\text { Main Results (SM vs. HET) } \\
\text { All Effects ORs if not other specified }\end{array}$ & Subgroup Differences & $\begin{array}{l}\text { Gender } \\
\text { Differences }\end{array}$ & Note \\
\hline \multicolumn{8}{|l|}{$\begin{array}{l}\text { Adolescent } \\
\text { Health Surveys }\end{array}$} \\
\hline $\begin{array}{l}\text { Konishi, } \\
\text { Saewyc, } \\
\text { Homma, \& } \\
\text { Poon (2013) b }\end{array}$ & $\begin{array}{l}21,708 \text { adolescents Canada, } \\
\text { BC } \\
\text { British Columbia Adolescent } \\
\text { Health Survey }\end{array}$ & $\begin{array}{l}3.4 \% \text { identified as } L G B \text {, based on } \\
\text { romantic attractions }\end{array}$ & $\begin{array}{l}\text { Past month binge drinking ( } \geq 6 \\
\text { days), marijuana use ( } \geq 20 \\
\text { days), } \geq 3 \text { post substance use } \\
\text { problems in past year }\end{array}$ & $\begin{array}{l}\text { All effects ss (boys, girls) } \\
\text { Binge drinking } 2.5,1.7 \\
\text { Marijuana } 1.4,1.7 \\
\text { Consequence: } 2.6,2.4\end{array}$ & None reported & Comparable & \\
\hline $\begin{array}{l}\text { Remafedi, } \\
\text { French, Story, } \\
\text { Resnick, \& } \\
\text { Blum (1998) }\end{array}$ & $\begin{array}{l}\text { 36,254 adolescents, USA, } \\
\text { mean age about } 15 \text { years, } \\
\text { stratified cluster sampling, } \\
\text { Minnesota Adolescent Health } \\
\text { Survey } 1998, \text { each LGB } \\
\text { participant was matched with } \\
\text { a HET participant }\end{array}$ & $\begin{array}{l}1 \% \text { of youth identified as LGB } \\
(0.7 \% \text { as } B, 0.3 \% \text { as } \mathrm{HO}) \\
\text { Questionnaire. }\end{array}$ & $\begin{array}{l}\text { Single item on SA (past year or } \\
\text { previously, coded as lifetime } \\
\text { attempts) }\end{array}$ & $\begin{array}{l}\text { Boys: } 7.1, \text { ss } \\
\text { Girls: } 1.5, \text { nss }\end{array}$ & None reported & $\begin{array}{l}\text { Larger SO } \\
\text { difference for men }\end{array}$ & $\begin{array}{l}\text { Young sample, } \\
\text { LGB women } \\
\text { tend to come } \\
\text { out later }\end{array}$ \\
\hline $\begin{array}{l}\text { Saewyc et al. } \\
(2007)^{b}\end{array}$ & $\begin{array}{l}\text { Several Regions and years: } \\
\text { Minnesota } 1986 \text { and } \\
\text { Minnesota student survey } \\
\text { 1992 + 1998, Seattle 1995+ } \\
\text { 1999, British Columbia 1992, } \\
\text { 1998, 2003, and National } \\
\text { American Indian Adolescent } \\
\text { Survey } 1990\end{array}$ & $\begin{array}{l}+++ \\
\text { Categorization based on sexual } \\
\text { attraction }(2-5 \% \text { SM), or behavior } \\
\text { in past year }(7-9 \%)\end{array}$ & SA in past year & $\begin{array}{l}\text { All effects median of RR across studies. } \\
\text { Girls: L 1.7, B 1.9, moHET 1.2. } \\
\text { Boys: G 3.4, B 3.9, moHET 1.3. }\end{array}$ & $\begin{array}{l}\text { Comparable effects } L G \text { and } \\
B \text { (only marginally } \\
\text { increases for B), smaller } \\
\text { effects for moHET, } \\
\text { especially for males. }\end{array}$ & $\begin{array}{l}\text { Larger effects for } \\
\text { GB males than LB } \\
\text { women, } \\
\text { comparable for } \\
\text { moHET }\end{array}$ & $\begin{array}{l}\text { Mixture of } \\
\text { Behavior and } \\
\text { Identity (see } \\
\text { Kann et al. } \\
2011 \text { for } \\
\text { related effects) }\end{array}$ \\
\hline $\begin{array}{l}\text { Boston Youth } \\
\text { Survey }\end{array}$ & $\begin{array}{l}\text { Stratified random sample } \\
\text { Representative sample of high } \\
\text { school students, USA. }\end{array}$ & & & & & & \\
\hline $\begin{array}{l}\text { Almeida, } \\
\text { Johnson, } \\
\text { Corliss, } \\
\text { Molnar, \& } \\
\text { Azrael (2009) }{ }^{b}\end{array}$ & 1,032 youth $13-19$ years-old & $\begin{array}{l}10 \% \text { identified as being } \\
\text { nonheterosexual or transgender }\end{array}$ & $\begin{array}{l}\text { Modified Depression Scale } \\
\text { (MDS), self-harm (suicidal intent } \\
\text { not assessed) in past year. }\end{array}$ & $\begin{array}{l}\text { Depression: men } d=0.4 \mathrm{ss} \text {, women } d=0.3 \mathrm{ss} \text {. } \\
\text { Self-harm: men } 20.3 \text {, women } 2.2 \text {, all ss }\end{array}$ & None reported & $\begin{array}{l}\text { Comparable for } \\
\text { depression, for } \\
\text { self-harm much } \\
\text { larger effect for } \\
\text { men }\end{array}$ & \\
\hline $\begin{array}{l}\text { Duncan \& } \\
\text { Hatzenbuehler } \\
(2014)^{b}\end{array}$ & $\begin{array}{l}1,198 \text { youth, mean age } 16.3 \\
\text { years-old. } \\
\text { Survey } 2008\end{array}$ & $\begin{array}{l}9 \% \text { identified as } \mathrm{moHE}, \mathrm{B}, \mathrm{moHO} \text {, } \\
\text { or } \mathrm{HO}, \text { or } \mathrm{Q} . \\
\text { Survey in classroom }\end{array}$ & $\begin{array}{l}\text { Past-month marijuana and other } \\
\text { illicit drug use similar to YRBS }\end{array}$ & $\begin{array}{l}\text { Marijuana use } 2.2 \mathrm{ss,} \\
\text { other illicit drug use } 4.8 \mathrm{ss} .\end{array}$ & None reported & None reported & \\
\hline
\end{tabular}


Online supplemental material to Plöderl \& Tremblay (2015). Mental health of sexual minorities. A systematic review. International Review of Psychiatry. http://dx.doi.org/10.3109/09540261.2015.1083949

\begin{tabular}{|c|c|c|c|c|c|c|c|}
\hline $\begin{array}{l}\text { Johnson et al. } \\
(2011)^{b}\end{array}$ & $\begin{array}{l}832 \text { girls, survey, 2008, } \\
\text { See Duncan \& Hatzenbuehler } \\
(2014)\end{array}$ & $\begin{array}{l}11.9 \% \text { identified as moHE }(3.4 \%) \\
\text { B }(4.8 \%), \text { moHO }(<1 \%), \mathrm{HO} \\
(1.1 \%) \text {, or Q }(1.6 \%) \text {. } \\
\text { Survey in classroom. }\end{array}$ & Modified Depression Scale & Ss effect for depression $d=0.4$ & None reported & - & \\
\hline \begin{tabular}{|l} 
Minnesota \\
Student Survey
\end{tabular} & $\begin{array}{l}\text { Survey of } 9 \text { th and } 12^{\text {th }} \\
\text { graders, USA }\end{array}$ & Survey in classroom & & & & & \\
\hline $\begin{array}{l}\text { M. E. } \\
\text { Eisenberg \& } \\
\text { Resnick (2006) } \\
\text { b }\end{array}$ & $\begin{array}{l}21,927 \text { sexually active youth, } \\
\text { survey } 2004\end{array}$ & $\begin{array}{l}10.3 \% \text { were classified as SM } \\
\text { because of having had "sexual } \\
\text { intercourse" in the past year with } \\
\text { at least one same-sex partner }\end{array}$ & SA in past year and lifetime & $\begin{array}{l}\text { Ss effects for lifetime SA } \\
\text { for boys } 2.5 \text { and girls } 2.6 \text {. }\end{array}$ & None reported & Comparable & $\begin{array}{l}\text { "intercourse" } \\
\text { as definition } \\
\text { perhaps not } \\
\text { appropriate }\end{array}$ \\
\hline $\begin{array}{l}\text { Taliaferro \& } \\
\text { Muehlenkamp } \\
(2014)\end{array}$ & $\begin{array}{l}70,722 \text { adolescents, } \\
\text { survey } 2010\end{array}$ & $\begin{array}{l}\text { Past year same-sex sexual } \\
\text { behavior (\# partners "intercourse") }\end{array}$ & SA in past year & $\begin{array}{l}\text { Comparison of those with SA and those without } \\
\text { any suicidality (ideation or attempts): } 1.4 \text { nss, } \\
\text { Comparing those with SA and those with suicide } \\
\text { ideation only: OR } 1.5, \text { nss. }\end{array}$ & None reported & None reported. & $\begin{array}{l}\text { "intercourse" } \\
\text { as definition, } \\
\text { and controlling } \\
\text { for depression/ } \\
\text { hopelessness. }\end{array}$ \\
\hline $\begin{array}{l}\text { Oregon } \\
\text { Healthy Teen } \\
\text { Survey }\end{array}$ & $\begin{array}{l}2006-2008, \text { random sample } \\
\text { from } 8^{\text {th }} \text { and } 11^{\text {th }} \text { graders, USA }\end{array}$ & & & & & & \\
\hline $\begin{array}{l}\text { Hatzenbuehler } \\
\text { (2011) and } \\
\text { Hatzenbuehler, } \\
\text { et al. (2014) }{ }^{\mathrm{b}}\end{array}$ & 31,852 youth & $\begin{array}{l}4.4 \% \text { identified as LGB }(0.9 \% \text { as } \\
\text { G, 3.3\% B), Q were excluded } \\
\text { Questionnaire }\end{array}$ & SA in past year & $\begin{array}{l}\text { Ss effects: LG 5.6, B } 6.4 \\
\text { (Note: slightly deviating results in } 2014 \text { paper: LG: } \\
\text { 5.9, B: } 6.7 \text { ) }\end{array}$ & $\begin{array}{l}\text { Slightly larger SO } \\
\text { difference for B compared } \\
\text { to LG }\end{array}$ & None reported & \\
\hline $\begin{array}{l}\text { Hatzenbuehler, } \\
\text { Pachankis, \& } \\
\text { Wolff }(2012)^{b}\end{array}$ & $\begin{array}{l}\text { See Hatzenbuehler, et al. } \\
(2014)\end{array}$ & See Hatzenbuehler, et al. (2014) & $\begin{array}{l}\# \text { of } 5 \text { symptoms of past year } \\
\text { alcohol abuse (present vs. } \\
\text { absent), \# days smoked in past } \\
\text { month (yes/no), depressive } \\
\text { period } \geq 2 \text { weeks in past year. }\end{array}$ & $\begin{array}{l}\text { Ss effect for } \\
\text { smoking }(d=0.5) \\
\text { drinking }(d=0.1) \\
\text { depression } 3.7\end{array}$ & None reported & None reported & $\begin{array}{l}\text { SO differences } \\
\text { at very young } \\
\text { age }\end{array}$ \\
\hline $\begin{array}{l}\text { Pittsburgh Girls } \\
\text { study }\end{array}$ & $\begin{array}{l}\text { Random household sample of } \\
2451 \text { girls in Pittsburgh, USA, } \\
\text { first wave } 2000 \text { when girls } \\
\text { were } 5-8 \text { years-old. SO } \\
\text { information assessed in } 2008 \text {. }\end{array}$ & $\begin{array}{l}\text { Home-based, face-to-face, } \\
\text { computer-assisted interviews (self- } \\
\text { reports). }\end{array}$ & & & & & \\
\hline $\begin{array}{l}\text { Marshal, } \\
\text { Sucato, et al. } \\
\text { (2012) }\end{array}$ & 527 girls, 17 years-old & $\begin{array}{l}6 \% \text { identified as LB }(25.8 \% \text { of } \\
\text { those as B) or as HET but with } \\
\text { current HO romantic attraction. }\end{array}$ & $\begin{array}{l}\text { Past year use of cigarette, } \\
\text { alcohol, binge drinking, } \\
\text { marijuana with the Nicotine, } \\
\text { Alcohol, and Drug Use scale; } \\
\text { conduct disorder and }\end{array}$ & $\begin{array}{l}\text { All substance use variables: } 2.4-2.9 \\
\text { Conduct disorder } d=0.5 \\
\text { Oppositional defiant disorder } d=0.4 \\
\text { Depression } d=0.7 \\
\text { Anxiety } d=0.6\end{array}$ & None reported & - & \\
\hline
\end{tabular}


Online supplemental material to Plöderl \& Tremblay (2015). Mental health of sexual minorities. A systematic review. International Review of Psychiatry. http://dx.doi.org/10.3109/09540261.2015.1083949

\begin{tabular}{|c|c|c|c|c|c|c|c|}
\hline & & & $\begin{array}{l}\text { oppositional defiant disorder } \\
\text { with the Adolescent Self- } \\
\text { Report Inventory. } \\
\text { Depression with the adolescent } \\
\text { Symptom Self-Report Inventory; } \\
\text { anxiety with the Screen for Child } \\
\text { Anxiety Related Emotional } \\
\text { Disorders (SCARED); } \\
\text { borderline personality with the } \\
\text { International Personality } \\
\text { Disorder Examination (IPDE), } \\
\text { SA (self harm) in past year. }\end{array}$ & $\begin{array}{l}\text { Borderline personality } d=0.4 \\
\text { SA } 7.2 \\
\text { All effects ss except for marijuana use. }\end{array}$ & & & \\
\hline $\begin{array}{l}\text { Marshal, } \\
\text { Dermody, } \\
\text { Shultz, et al. } \\
(2013)^{\text {b }}\end{array}$ & $\begin{array}{l}2064 \text { girls, } 16-19 \text { years-old } \\
\text { from Wave } 11 .\end{array}$ & $\begin{array}{l}8.3 \% \text { identified as LB }(81.5 \% \text { of } \\
\text { them as } B) \text {. }\end{array}$ & $\begin{array}{l}\text { See Marshal et al. (2012). } \\
\text { Self-injury (SA?) in past year } \\
\text { was assesses with the item from } \\
\text { SCID-I }\end{array}$ & $\begin{array}{l}\text { All effects ss:substance (alcohol } 2.7 \text {, binge } \\
\text { drinking } 3.0 \text {, cigarette use } 5.2 \text {, marijuana } 3.6 \text { ). } \\
\text { Effect sizes n.a. for conduct disorder and } \\
\text { oppositional defiant disorder. } \\
\text { Depression, anxiety ( } d=0.6) \text {. } \\
\text { Borderline personality }(d=0.4) \text {. } \\
\text { Self-injury (SA?): } 6.8\end{array}$ & None reported & - & \\
\hline $\begin{array}{l}\text { Birkett, } \\
\text { Espelage, \& } \\
\text { Koenig (2009) }\end{array}$ & $\begin{array}{l}\text { 7,376 youth, USA, } \\
\text { mostly } 12-14 \text { years-old, } \\
80 \% \text { of Dane County Middle } \\
\text { School, } 7-12 \text { graders, }\end{array}$ & $\begin{array}{l}\text { Question on being confused about } \\
\text { being LGB. Those not confused } \\
\text { because being sure to be straight } \\
\text { ( } 75.2 \%) \text { or LGB were categorized } \\
\text { HET or LGB }(10.5 \%) \text {, those being } \\
\text { confused at least sometimes were } \\
\text { categorized as Q }(4.6 \%) \text {. }\end{array}$ & $\begin{array}{l}\text { 11-item scale on } \\
\text { alcohol/marijuana use } \\
\text { (combined), } \\
\text { item on suicide } \\
\text { ideation/depression in past } \\
\text { month combined. }\end{array}$ & $\begin{array}{l}\text { Ss effects (all Cohen's d): } \\
\text { alcohol/marijuana: LGB } 0.2, \text { Q } 0.6 \\
\text { depression/suicidality: LGB } 0.4, \text { Q } 0.7\end{array}$ & $\begin{array}{l}\text { Higher levels for } Q \\
\text { compared to LGB studens }\end{array}$ & None reported & \\
\hline $\begin{array}{l}\text { Bos, Sandfort, } \\
\text { de Bruyn, \& } \\
\text { Hakvoort } \\
\text { (2008) }\end{array}$ & $\begin{array}{l}866 \text { high school students, ( } 479 \\
\text { boys, } 387 \text { girls, (mean age } \\
13.6 \text { years old), Netherlands, } \\
2004 \text {, representative sample }\end{array}$ & $\begin{array}{l}8.5 \% \text { had some feelings of same- } \\
\text { sex attraction (Likert scaled, } 5 \\
\text { options from never to very often). } \\
\text { Computer based questionnaire. }\end{array}$ & $\begin{array}{l}\text { Depression with the General } \\
\text { Health Questionnaire }\end{array}$ & $\begin{array}{l}\text { Ss correlation of } \mathrm{HO} \text { attraction with depression, } \\
\mathrm{r}=.26\end{array}$ & None reported & None reported & \\
\hline $\begin{array}{l}\text { Collier, Bos, \& } \\
\text { Sandfort } \\
\text { (2013) }\end{array}$ & $\begin{array}{l}513 \text { adolescents, } 11-17 \text { years- } \\
\text { old; from } 8 \text { schools, } \\
\text { Amsterdam area, Netherlands }\end{array}$ & $\begin{array}{l}11.1 \% \text { of girls and } 10.8 \% \text { of boys } \\
\text { reported some } \mathrm{HO} \text { attractions } \\
\text { (Likert scale: never to very often) }\end{array}$ & $\begin{array}{l}\text { Psychological Distress (Short } \\
\text { Version of the Brief Symptom } \\
\text { Inventory) }\end{array}$ & $\begin{array}{l}\text { Ss effect, } d=0.5 \\
\text { very strong evidence, } B F=19.9\end{array}$ & None reported & & \\
\hline $\begin{array}{l}\text { Denny et al. } \\
\text { (2014) }\end{array}$ & $\begin{array}{l}\text { Adolescents, } 13-17 \text { years old, } \\
\text { New Zealand high school, } \\
2007 \text {, random sampling within } \\
\text { schools grades 9-13. }\end{array}$ & $\begin{array}{l}0.9 \% \text { reported } \mathrm{HO} \text { attraction, } 3.5 \% \\
B(\mathrm{Q} \text { and not attracted excluded). } \\
\text { ACASI. }\end{array}$ & $\begin{array}{l}\text { High depressive symptoms } \\
\text { (cutoff not given) with Reynolds } \\
\text { Adolescent Depression Scale } \\
\text { (RAD-SF), SA in past year. }\end{array}$ & $\begin{array}{l}\text { Effects all ss. } \\
\text { Depression: HO 2.5, B } 4.5 \\
\text { SA: HO 3.9, B } 11.2\end{array}$ & $\begin{array}{l}\text { Clearly larger effect for B } \\
\text { compared to } \mathrm{HO}\end{array}$ & None reported & \\
\hline Fleming, & 9,570 Adolescents, mostly $13-$ & Percentage not given, not clear if & SA in past year & Ss effect 1.8, & None reported & None reported & Likely under- \\
\hline
\end{tabular}


Online supplemental material to Plöderl \& Tremblay (2015). Mental health of sexual minorities. A systematic review. International Review of Psychiatry. http://dx.doi.org/10.3109/09540261.2015.1083949

\begin{tabular}{|c|c|c|c|c|c|c|c|}
\hline \begin{tabular}{|l|} 
Merry, \\
Robinson, \\
Denny, \& \\
Watson (2007)
\end{tabular} & $\begin{array}{l}16 \text { years, New Zealand } \\
\text { Adolesc. Health Survey, } \\
\text { Random sample }\end{array}$ & $\begin{array}{l}\text { SM classification based on sexual } \\
\text { attraction or self-identification, } \\
\text { Multi-Media CASI. }\end{array}$ & & $\begin{array}{l}\text { in multivariate model (+) including depression, } \\
\text { anxiety, alcohol, violence etc. as covariates. }\end{array}$ & & & \begin{tabular}{|l} 
estimations, \\
bivariate \\
association not \\
given
\end{tabular} \\
\hline $\begin{array}{l}\text { Hagger- } \\
\text { Johnson et al. } \\
\text { (2013) }\end{array}$ & $\begin{array}{l}\text { 7,698 youth 18-19 years-old, } \\
\text { UK, Longitudinal Study of } \\
\text { Young People in England } \\
\text { (LSYPE) } \\
\text { Wave } 6 \text { (2009) }\end{array}$ & $\begin{array}{l}+++ \\
1.1 \% \text { identified as LG, } 1.9 \% \text { as B, } \\
\text { Home visit, web-questionnaire, } \\
\text { and telephone interview }\end{array}$ & $\begin{array}{l}\text { Smoking status (yes } / \mathrm{no} \text { ), past } \\
\text { year drinking (> } 2 \text { times a week } \\
\text { vs. less), risky drinking (getting } \\
\text { drunk }>52 \text { times a year ) }\end{array}$ & $\begin{array}{l}\text { Smoking, G, L, B men and B women: 1.9 - 2.4, all } \\
\text { ss except B males. } \\
\text { Drinking: G } 1.9 \text { ss, L } 2.1 \text { nss, B women 1.3, nss, B } \\
\text { men 1.0, nss. } \\
\text { Risky drinking: G men } 2.1 \text { ss, L } 1.3 \text { nss, } \\
\text { B women } 1.4 \text { nss, B men } 0.7 \text { nss. }\end{array}$ & $\begin{array}{l}\text { Larger SO difference for } L G \\
\text { than compared to } B \text {. } \\
G \text { men at risk for risky } \\
\text { drinking but protective } \\
\text { effect for B men. }\end{array}$ & Comparable & \\
\hline $\begin{array}{l}\text { Hatzenbuehler, } \\
\text { Corbin, \& } \\
\text { Fromme (2008) }\end{array}$ & $\begin{array}{l}\text { 2,220 High School students, } \\
\text { USA, 17-19 years-old, USA, } \\
\text { Texas, assessed last year of } \\
\text { high school and in the first two } \\
\text { semesters in University, } \\
\text { random sample. }\end{array}$ & $\begin{array}{l}5.0 \% \text { were classified as LGBQ } \\
\text { either because of identification as } \\
\text { such in any of the three time points } \\
\text { or because of having had same- } \\
\text { sex sexual contacts in the past } 3 \\
\text { months }\end{array}$ & $\begin{array}{l}\text { Alcohol use composite } \\
\text { measures out of \# drinking days } \\
\text { in average week, average \# } \\
\text { drinks on drinking day, \# } \\
\text { drinking until intoxication and \# } \\
\text { binge drinking. }\end{array}$ & $\begin{array}{l}\text { All effects Cohen's d: } \\
\text { Girls: } \\
\text { \# drinking days: high school } 0.5 \mathrm{ss}, 0.3 \mathrm{nss} \\
\text { \# drinking until intoxication: } 0.2 \mathrm{ss}, 0.2 \mathrm{nss} \\
\text { \# binge drinking: } 0.2 \mathrm{ss}, 0.2 \mathrm{nss} \\
\text { Boys : } \\
\text { \# drinking days: high school } 0.1 \mathrm{ss}, 0.1 \mathrm{nss} \\
\text { \# drinking until intoxication: }-0.1 \mathrm{ss}, 0.1 \mathrm{ss} \\
\text { \# binge drinking: }-0.1 \text { nss, } 0.0 \mathrm{nss}\end{array}$ & None reported & $\begin{array}{l}\text { Changing over } \\
\text { time, ss effect for } \\
\text { girls in high } \\
\text { school but not in } \\
\text { university, } \\
\text { reversed among } \\
\text { boys. }\end{array}$ & \\
\hline $\begin{array}{l}\text { Hatzenbuehler, } \\
\text { McLaughlin, \& } \\
\text { Nolen- } \\
\text { Hoeksema } \\
\text { (2008) }\end{array}$ & $\begin{array}{l}\text { 1,071 adolescents, 11-14 } \\
\text { years-old, USA, from two } \\
\text { middle schools in Connecticut, } \\
2005 \text {, follow up after } 7 \text { months } \\
\text { in } 2006\end{array}$ & $\begin{array}{l}2.3 \% \text { ever having been same sex } \\
\text { or both sex romantic-attracted, } \\
\text { questionnaire }\end{array}$ & $\begin{array}{l}\text { Children's depression inventory } \\
\text { (CDI), multidimensional anxiety } \\
\text { scale for children (MASC) }\end{array}$ & $\begin{array}{l}\text { Ss effects (all Cohen's d) } \\
\text { Depression: Wave } 1: 0.5 \text {, Wave 2: } 0.6 \\
\text { Anxiety: } 0.4 \text { and } 0.6\end{array}$ & None reported & None reported & \\
\hline $\begin{array}{l}\text { Hidaka et al. } \\
\text { (2008) }\end{array}$ & $\begin{array}{l}\text { 2,095 youth, 14-24 years-old } \\
\text { (mean } 19.7 \text { males, } 18.9 \\
\text { females), Japan, Osaka, } \\
\text { recruited with street intercept } \\
\text { method }\end{array}$ & $\begin{array}{l}3.7 \text { identified as LGB, } \\
\text { Questionnaire }\end{array}$ & Lifetime SA & $\begin{array}{l}\text { Males: } 6.6 \mathrm{ss} \\
\text { Females: } 2.1 \mathrm{nss}\end{array}$ & None reported & None reported & \\
\hline $\begin{array}{l}\text { Lampinen, } \\
\text { McGhee, \& } \\
\text { Martin (2006) }\end{array}$ & $\begin{array}{l}590 \text { adolescents, } \\
\text { CA, Victoria and Vancouver, } \\
\text { randomly sampled from } \\
\text { selected high schools, grades } \\
\text { 9-12. }\end{array}$ & $\begin{array}{l}2.54 \% \text { identified as } G \text { and } B \text {. One } \\
\text { categorical item measuring the } \\
\text { orientation }\end{array}$ & $\begin{array}{l}\text { Self-reported depression } \geq 1 \\
\text { month, SA, } \\
\text { Past year substance use. }\end{array}$ & $\begin{array}{l}\text { Ss effects for depression } 13.5 \text { and SA } 17.4 \text {. } \\
\text { Ss effects for substance use (except alcohol } 1.2 \\
\text { and GHB } 2.3) \text {, marijuana } 4.5 \text {, cigarettes } 3.3 \text {, } \\
\text { mushrooms 5.0, ecstasy } 4.0 \text {, cocaine } 5.4 \text {, } \\
\text { ketamine } 4.5, \text { crystal meth } 20.5 \text {, heroine and } \\
\text { peyote n.a. }\end{array}$ & None reported & & \\
\hline $\begin{array}{l}\text { Lucassen et al. } \\
(2014)\end{array}$ & $\begin{array}{l}\text { 9,011 (2001), 8,002 (2007), } \\
\text { 8,167 (2012) youth, New } \\
\text { Zealand, High school }\end{array}$ & $\begin{array}{l}\text { Around } 6 \% \text { in all waves }(5.9 \% \text { - } \\
6.1 \%) \text { were classified as SM, } \\
\text { based on sexual attraction. }\end{array}$ & $\begin{array}{l}\text { Depression with RADS (using } \\
\text { clinical threshold } \geq 28 \text { ), binge } \\
\text { drinking in past } 4 \text { weeks, }\end{array}$ & $\begin{array}{l}\text { Depression over the three waves }(2001,2007 \text {, } \\
\text { 2012): } 2.4,3.6,3.7 \text {, all ss. } \\
\text { Drinking: close to } 1.1 \text { and nss in all three waves. }\end{array}$ & None reported & $\begin{array}{l}\text { Larger effect } \\
\text { among males for } \\
\text { SA but not for }\end{array}$ & $\begin{array}{l}\text { Increasing } \\
\text { effects over } \\
\text { time for SA }\end{array}$ \\
\hline
\end{tabular}


Online supplemental material to Plöderl \& Tremblay (2015). Mental health of sexual minorities. A systematic review. International Review of Psychiatry. http://dx.doi.org/10.3109/09540261.2015.1083949

\begin{tabular}{|c|c|c|c|c|c|c|c|}
\hline & $\begin{array}{l}\text { students, Youth2000 surveys, } \\
\text { representative sample }\end{array}$ & Computer assisted survey & SA in past year. & SA: males: $2.9,7.7,5.6$; females: $1.9,4.0,3.7$ & & $\begin{array}{l}\text { depression and } \\
\text { binge drinking. }\end{array}$ & \\
\hline $\begin{array}{l}\text { Madge et al. } \\
(2011)\end{array}$ & $\begin{array}{l}30,477 \text { adolescents from six } \\
\text { European countries, majority } \\
15-16 \text { years-old; data from } \\
\text { CASE study, random sample }\end{array}$ & $\begin{array}{l}\text { None reported: } 5.7 \% \text { worries about } \\
\text { sexual orientation (subcategory of } \\
\text { live events) }\end{array}$ & $\begin{array}{l}\text { Deliberate self harm in past year } \\
\text { (single or multiple combined). }\end{array}$ & Ss effect 3.7 & None reported & None reported & $\begin{array}{l}\text { Problematic } \\
\text { definition of SO }\end{array}$ \\
\hline $\begin{array}{l}\text { Martin-Storey } \\
\& \text { Crosnoe } \\
(2012)\end{array}$ & $\begin{array}{l}957 \text { adolescents, NICHD } \\
\text { study of early child care and } \\
\text { youth development } \\
\text { (SECCYD), sampled via } \\
\text { parents of newborns, followed } \\
\text { up until age } 15 .\end{array}$ & $\begin{array}{l}\text { LGB No percentage reported. } \\
\text { Single item: Preferred romantic } \\
\text { partners plus one item on } \\
\text { harassment due to sexual minority } \\
\text { status }\end{array}$ & $\begin{array}{l}\text { Depression (Short Form of the } \\
\text { Child Depression inventory) }\end{array}$ & Ss effect for depression, $d=0.4$ & None reported & $\begin{array}{l}\text { Gender } \\
\text { considered as } \\
\text { control variable }\end{array}$ & $\begin{array}{l}\text { Mediation } \\
\text { analysis }\end{array}$ \\
\hline $\begin{array}{l}\text { McMahon, } \\
\text { Reulbach, } \\
\text { Keeley, Perry, } \\
\text { \& Arensman } \\
(2012)\end{array}$ & $\begin{array}{l}\text { 1,870 boys, } 15-17 \text { years-old, } \\
\text { Ireland, Southern region of the } \\
\text { Health Service Executive } \\
\text { Child and Adolescent Self } \\
\text { Harm in Europe (CASE) study }\end{array}$ & $\begin{array}{l}\text { Worries about sexual orientation, } \\
\% \text { not given. } \\
\text { Questionnaire in Classroom }\end{array}$ & Lifetime self harm & $\begin{array}{l}\text { Ss effect among those with lifetime school bullying } \\
\text { (5.6) and without (4.7). }\end{array}$ & - & - & $\begin{array}{l}\text { Problematic } \\
\text { definition of } \\
\text { sexual } \\
\text { orientation }\end{array}$ \\
\hline $\begin{array}{l}\text { O'Connor, } \\
\text { Rasmussen, \& } \\
\text { Hawton (2009) }\end{array}$ & $\begin{array}{l}737 \text { adolescents, } 15-16 \text { years- } \\
\text { old at first assessment, } \\
500 \text { adolescents at follow up } 6 \\
\text { months later, Scotland } \\
\text { See O'Conner } 2006\end{array}$ & $\begin{array}{l}3.35 \% \text { reported sexual orientation } \\
\text { worries }\end{array}$ & $\begin{array}{l}\text { Self harm in follow up period } \\
\text { (past } 6 \text { months) }\end{array}$ & $\begin{array}{l}\text { Ss effect for repeat DSH within follow up period } \\
(9.6) \\
\text { Nss for first time DSH in follow up period (OR = } \\
2.2)\end{array}$ & None reported & None reported & $\begin{array}{l}\text { Problematic } \\
\text { definition of } \\
\text { sexual } \\
\text { orientation }\end{array}$ \\
\hline $\begin{array}{l}\text { O'Connor, } \\
\text { Rasmussen, } \\
\text { Miles, \& } \\
\text { Hawton (2009) }\end{array}$ & $\begin{array}{l}\text { 2,008 adolescents, Glasgow, } \\
\text { Scotland, random sample }\end{array}$ & $\begin{array}{l}\text { No percentage or number given. } \\
\text { Sexual orientation worries }\end{array}$ & $\begin{array}{l}\text { Lifetime deliberate self-harm } \\
\text { (DSH) }\end{array}$ & $\begin{array}{l}\text { Girls: } 2.6 \mathrm{ss} \text {, Boys } 3.8 \mathrm{ss} \text {. } \\
\text { CAVE: ORs adjusted for many risk factors, thus } \\
\text { OR's are likely underestimations. }\end{array}$ & None reported & See main results & $\begin{array}{l}\text { Problematic } \\
\text { SO-definition, } \\
\text { adjusted ORs }\end{array}$ \\
\hline $\begin{array}{l}\text { O'Connor, } \\
\text { Rasmussen, \& } \\
\text { Hawton (2014) }\end{array}$ & $\begin{array}{l}3,596 \text { adolescents, mostly } 15- \\
16 \text { years-old (mean } 15), \\
\text { Northern Ireland Lifestyle and } \\
\text { Coping Survey, Random } \\
\text { sample of high schools }\end{array}$ & $\begin{array}{l}4.2 \% \text { of girls and } 6.3 \% \text { of boys had } \\
\text { worries about their sexual } \\
\text { orientation. } \\
\text { Questionnaire in Classroom }\end{array}$ & Lifetime self harm & Ss among girls (4.0) and boys (9.9). & - & $\begin{array}{l}\text { Clearly larger } \\
\text { effect among } \\
\text { male participants }\end{array}$ & $\begin{array}{l}\text { Problematic } \\
\text { definition of } \\
\text { sexual } \\
\text { orientation }\end{array}$ \\
\hline $\begin{array}{l}\text { Ortiz- } \\
\text { Hernandez, } \\
\text { Tello, \& Valdes } \\
(2009)\end{array}$ & 12,796 adolescents, Mexico & $\begin{array}{l}11.6 \% \text { reported some lifetime } \\
\text { homosexual attraction (falling in } \\
\text { love). } \\
0.9 \% \text { identified as LG, } 0.7 \text { as B. } \\
1.4 \% \text { reported lifetime same-sex } \\
\text { sexual contacts. }\end{array}$ & $\begin{array}{l}\text { Current cigarette use, smoking } \\
\geq 6 \text { cigarettes a day, } \\
\text { Current alcohol use, } \\
\text { intake of } \geq 6 \text { drink per week }\end{array}$ & $\begin{array}{l}\text { Attraction: Smoking, } 1.6-2.8 \text {, ss except }>6 \text { drinks } \\
\text { Identity: smoking } 2.3 \mathrm{ss}, \geq 6 \text { cigarettes } 5.5 \mathrm{ss,} \\
\text { drinking } 2.2, \geq 6 \text { drinks } 1.0 \text { nss. } \\
\text { Behavior: smoking n.a., } \geq 6 \text { cigarettes } 3.5 \mathrm{ss,} \\
\text { drinking n.a., } \geq 6 \text { drinks } 1.3 \text { nss. } \\
\text { For men, reversed effects for current alcohol use } \\
(0.6, \mathrm{ss}) \text { but not for women }(3.3, \mathrm{ss}) \text {. }\end{array}$ & Not reported & $\begin{array}{l}\text { LG behaving } \\
\text { women were } \\
\text { more likely to } \\
\text { consume alcohol } \\
\text { No ss significant } \\
\text { interactions with } \\
\text { gender. }\end{array}$ & \\
\hline
\end{tabular}


Online supplemental material to Plöderl \& Tremblay (2015). Mental health of sexual minorities. A systematic review. International Review of Psychiatry. http://dx.doi.org/100.3109/09540261.2015.1083949

\begin{tabular}{|c|c|c|c|c|c|c|c|}
\hline $\begin{array}{l}\text { Pesola, } \\
\text { Shelton, \& van } \\
\text { den Bree } \\
(2014)\end{array}$ & $\begin{array}{l}\text { 3,710 adolescents, UK, } 15-18 \\
\text { years-old, Avon Longitudinal } \\
\text { Study of Parents and Children, } \\
1991 / 1992 \text { cohort of children } \\
\text { (pregnant mothers in Avon). }\end{array}$ & $\begin{array}{l}12 \% \text { identified as SM at age } 15 \\
(9.3 \% \text { moHET, } 1.6 \% \text { B, } 0.6 \% \\
\text { moHO, } 0.3 \% \mathrm{HO})\end{array}$ & $\begin{array}{l}\text { Alcohol problems (AUDIT) at } \\
\text { age } 16 \text { and } 18 \text {, depression with } \\
\text { the SMFQ at age } 14 \text { and } 16 .\end{array}$ & $\begin{array}{l}\text { Depression: age } 14 \text { and } 16: d^{\prime} s=0.5 \\
\text { Alcohol age } 16: d=0.3 \text {, age } 18 d=0.1\end{array}$ & None reported & $\begin{array}{l}\text { Comparable } \\
\text { effects. }\end{array}$ & \\
\hline $\begin{array}{l}\text { Poteat, } \\
\text { Aragon, } \\
\text { Espelage, \& } \\
\text { Koenig (2009) }\end{array}$ & $\begin{array}{l}\text { 14,439 youth, USA, 14-19 } \\
\text { years-old (mean 15.9), high } \\
\text { schools of Midwestern county. }\end{array}$ & $\begin{array}{l}7.4 \% \text { were LGB because they } \\
\text { stated not being confused about } \\
\text { being LGB, those who were } \\
\text { confused to some degree were } \\
\text { classified as Q }(6.5 \%) \text {. }\end{array}$ & $\begin{array}{l}\text { 10-item scale on frequency of } \\
\text { substance/alcohol use in past } \\
\text { year. } \\
\text { Feeling depressed/suicidal }\end{array}$ & $\begin{array}{l}\text { All effects Cohen's d: } \\
\text { Depression/suicidal (white, racial minority) } \\
\text { Male LGB: } 0.1,0.2, Q: 0.5,0.5 \\
\text { Female LGB: } 0.3 ., 0.2, Q: 0.8,0.4 \\
\text { Substance use (white, racial minority) } \\
\text { male LGB: } 0.2,0.2, Q: 0.6,0.8 \\
\text { female LGB: } 0.4,0.4, Q: 0.4,0.4\end{array}$ & Q larger effects than LGB. & $\begin{array}{l}\text { Depression: } \\
\text { comparable } \\
\text { except LGB: } \\
\text { female>male } \\
\text { Substance abuse: } \\
\text { LGB } \\
\text { female>male, } \\
\text { reversed for Q }\end{array}$ & \\
\hline $\begin{array}{l}\text { Orenstein } \\
\text { (2001) }\end{array}$ & $\begin{array}{l}\text { 2,946 youth, USA, grade } 9 \text { to } \\
12 \text { from a High-School in } \\
\text { Massachusetts, two cohorts } \\
(1992,1994),\end{array}$ & $\begin{array}{l}7.3 \% \text { ever had HO romantic } \\
\text { attractions, } 2.2 \% \text { identified as } \\
\text { LGB, } 2.4 \% \text { had } \mathrm{HO} \text { contacts in the } \\
\text { past year, } 5.4 \text { worried about being } \\
\text { LGB in the past year and } 5.1 \% \\
\text { worried about being treated } \\
\text { differently because of being LGB. } \\
\text { For the analyses, responses to the } \\
5 \text { items were added up. }\end{array}$ & $\begin{array}{l}\text { Single items on Alcohol and } \\
\text { Drug use in the past month and } \\
\text { lifetime. Tabled results for drug } \\
\text { and alcohol use given for } \\
\text { heterosexual students and 'SM' } \\
\text { in three categories: Those } \\
\text { having one, two, and three to } \\
\text { five indication that one is SM. }\end{array}$ & 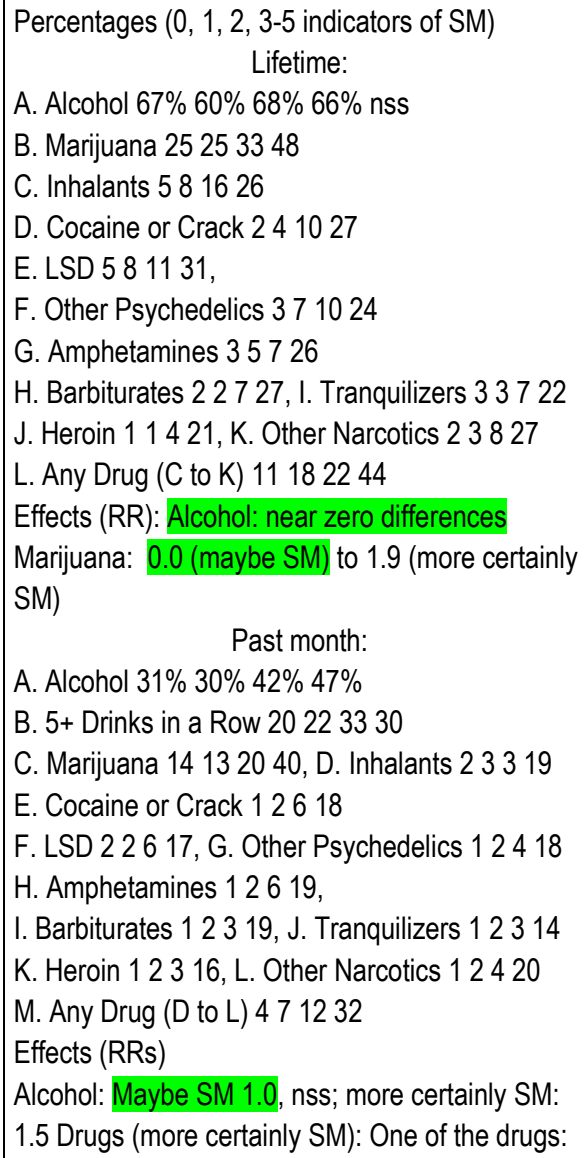 & $\begin{array}{l}\text { Those who are certainly } \\
\text { SM (having more indicators } \\
\text { of SM) are at higher risk } \\
\text { than those with only few } \\
\text { indicators. }\end{array}$ & $\begin{array}{l}\text { Basic pattern is } \\
\text { similar for both } \\
\text { genders, but SM } \\
\text { females more } \\
\text { likely use alcohol } \\
\text { (53 vs. } 37 \%) \text { but } \\
\text { no such } \\
\text { difference in } \\
\text { heavy drinking. } \\
\text { G males more } \\
\text { likely use hard } \\
\text { drugs ( } 41 \text { vs. } \\
24 \%) \text {. }\end{array}$ & $\begin{array}{l}\text { Indicator of SM } \\
\text { includes } \\
\text { worrying about } \\
\text { being LG and } \\
\text { worrying about } \\
\text { being treated } \\
\text { differently of } \\
\text { being LGB }\end{array}$ \\
\hline
\end{tabular}


Online supplemental material to Plöderl \& Tremblay (2015). Mental health of sexual minorities. A systematic review. International Review of Psychiatry. http://dx.doi.org/10.3109/09540261.2015.1083949

\begin{tabular}{|c|c|c|c|c|c|c|c|}
\hline & & & & $\begin{array}{l}\text { 19.0 Any one of the } 9+\text { drugs: } 2.6, \text { Marijuana: } \\
\text { Relative Risk: } 0.0 \text { (maybe SM) to } 1.9 \text { (more } \\
\text { certainly SM) }\end{array}$ & & & \\
\hline $\begin{array}{l}\text { Renaud, } \\
\text { Berlim, Begolli, } \\
\text { McGirr, \& } \\
\text { Turecki (2010) }\end{array}$ & $\begin{array}{l}55 \text { consecutive youth suicides, } \\
11-18 \text { years-old, Quebec, } \\
\text { Canada, matched with } 55 \\
\text { living controls }\end{array}$ & $\begin{array}{l}\text { Among suicides, } 7.3 \% \text { were } \\
\text { identified as } \mathrm{SM} \text {, and } 0 \% \text { among } \\
\text { living controls. Psychological } \\
\text { autopsy method (indirect } \\
\text { information from relatives/friends). }\end{array}$ & Suicides & $\begin{array}{l}\text { SM are overrepresented among suicide victims, } \\
\text { Bayesian OR = } 7.3 \text { and classical ss depends on } \\
\text { statistical test used (see Plöderl et al., 2013). }\end{array}$ & Not reported & $\begin{array}{l}\text { Effect among } \\
\text { males, not among } \\
\text { females }\end{array}$ & $\begin{array}{l}\text { Incorrect } \\
\text { interpretation } \\
\text { of findings in } \\
\text { original study. }\end{array}$ \\
\hline $\begin{array}{l}\text { T. G. Sandfort, } \\
\text { Bos, Collier, \& } \\
\text { Metselaar } \\
\text { (2010) }\end{array}$ & $\begin{array}{l}518 \text { adolescent } 12-15 \text { years- } \\
\text { old, from } 72 \text { secondary } \\
\text { schools in Amsterdam. }\end{array}$ & $\begin{array}{l}11 \% \text { reported some degree of } \\
\text { sexual attraction (Likert scaled } \\
\text { item). Computer Survey (and } \\
\text { paper in few cases). }\end{array}$ & $\begin{array}{l}\text { Mental Health assessed with the } \\
\text { Brief Symptom Inventory }\end{array}$ & $\begin{array}{l}\text { SS effect in multivariate analysis (with } \\
\text { confounders). }\end{array}$ & Not reported & Not reported & $\begin{array}{l}\text { Univariate } \\
\text { results n.a. }\end{array}$ \\
\hline $\begin{array}{l}\text { Shaffer, Fisher, } \\
\text { Hicks, Parides, } \\
\text { \& Gould (1995) }\end{array}$ & $\begin{array}{l}120 \text { consecutive youth } \\
\text { suicides, up to } 19 \text { years-old, } \\
\text { from New York City area, } \\
\text { USA, matched for analysis } \\
\text { with } 147 \text { living controls. }\end{array}$ & $\begin{array}{l}\text { Among suicides, } 2.5 \% \text { were } \\
\text { identified as } \mathrm{SM} \text {, and } 0 \% \text { among } \\
\text { living controls. Psychological } \\
\text { autopsy method (indirect } \\
\text { information from relatives/friends). }\end{array}$ & Suicides & $\begin{array}{l}\text { SM are overrepresented among suicide victims. } \\
\text { Bayesian OR = } 6.7 \text { and classical ss depends on } \\
\text { statistical test used (Plöderl et al. (2013)) }\end{array}$ & Not reported & $\begin{array}{l}\text { Effect among } \\
\text { males, not among } \\
\text { females }\end{array}$ & $\begin{array}{l}\text { Incorrect } \\
\text { interpretation } \\
\text { of findings in } \\
\text { original study. }\end{array}$ \\
\hline $\begin{array}{l}\text { Wang, et al. } \\
\text { (2012) }\end{array}$ & $\begin{array}{l}4044 \text { males, } 16-20 \text { years-old, } \\
\text { second Swiss Multicenter } \\
\text { Adolescent Survey on Health } \\
\text { (SMASH, 2002), post- } \\
\text { compulsory schooling, random } \\
\text { cluster sampling from most } \\
\text { cantons. }\end{array}$ & $\begin{array}{l}\text { SM based on attraction. } \\
\text { Results reported for "homosexual / } \\
\text { bisexual" (1.6\%) vs."only } \\
\text { heterosexual" (72.5\%), } 72.5 \% \\
\text { only heterosexual, } 23.3 \% \text { moHET, } \\
0.8 \% \text { B, } 0.4 \% \text { moHO, } 0.4 \% \text { only } \\
\text { HO, and } 2.6 \% \text { nonresponse }\end{array}$ & SA lifetime and past year & $\begin{array}{l}\text { SA: } \\
\text { Lifetime: OR: } 5.3, \text { ss. } \\
\text { Past Year: OR: 2.1, nss. }\end{array}$ & $\begin{array}{l}\text { No results reported, } \\
\text { but moHET reported as } \\
\text { being comparable with } \\
\text { HET. }\end{array}$ & - & \\
\hline $\begin{array}{l}\text { Zhao, Montoro, } \\
\text { Igartua, \& } \\
\text { Thombs (2010) }\end{array}$ & $\begin{array}{l}1,85614+\text { years-old from high } \\
\text { school in Montreal, Canada, }\end{array}$ & $\begin{array}{l}\text { 3.1\% identified as LGB, } 3.2 \% \text { Q, } \\
\text { and } 6.2 \text { as HET but had some } \\
\text { HO/B behavior or attraction (HET- } \\
\text { HO) }\end{array}$ & $\begin{array}{l}\text { Felt depression in past year ( } \geq 2 \\
\text { weeks that stopped daily } \\
\text { activities), past month smoking, } \\
\text { alcohol, marijuana, and lifetime } \\
\text { hard drugs } \\
\text { SA in past year }\end{array}$ & $\begin{array}{l}\text { All effects RRs (LGB, Q, HET-HO) } \\
\text { Depressed mood: } 1.9 \mathrm{ss}, 1.4 \mathrm{nss}, 1.5 \mathrm{nss} \\
\text { Smoking: } 2.2 \mathrm{ss}, 2.0 \mathrm{ss}, 2.2 \mathrm{ss} \\
\text { Alcohol: } 1.4 \mathrm{ss}, 0.9 \mathrm{nss}, 1.2 \mathrm{nss} \\
\text { Marijuana: } 1.9 \mathrm{ss}, 1.4 \mathrm{nss}, 1.3 \mathrm{nss} \\
\text { Hard drug use: } 2.8 \mathrm{ss}, 3.8 \mathrm{ss}, 2.1 \mathrm{ss} . \\
\text { SA: } 2.2 \mathrm{ss}, 1.6 \mathrm{nss}, 1.0 \mathrm{nss}\end{array}$ & $\begin{array}{l}\text { Comparable effects among } \\
\text { the subgroups except for } \\
\text { SA, where HET-HO and a } \\
\text { zero effect }\end{array}$ & Not reported & \\
\hline
\end{tabular}




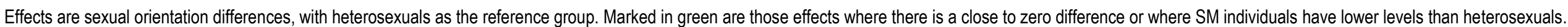

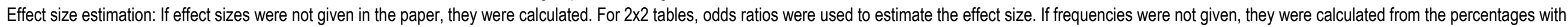

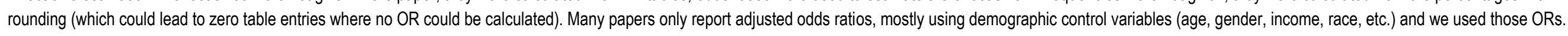

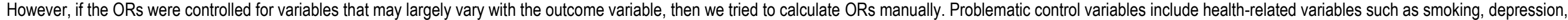

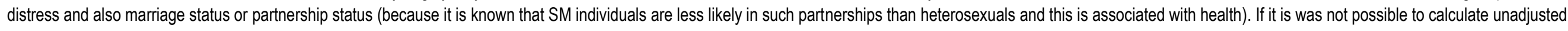
ORs, than we either contacted the author or we used the adjusted ORs but noted this in the table.

Risk ratios (RR) are calculated instead of ORs in some instances for convenience - for rare events ORs and RRs are usually comparable. However, we are planning to exchange the RRs with ORs for consistency.

For the comparisons of two means, we calculated Cohen's $d$ or the Bayes Factor, using the results from $t$-Test according to http://pcl.missouri.edu/bf-two-sample

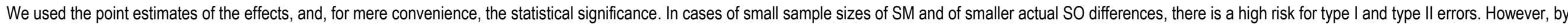
nature of random sampling, such errors cancel out when forming an overall impression. Thus, because of the multitude of studies, the overall impression will be valid.

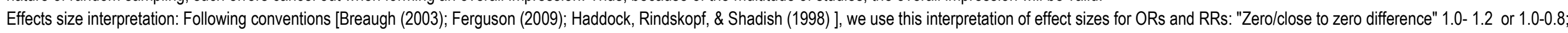
small 1.3-2.0 or 0.7-0.5; medium 2.1-3.5 or 0.4-0.3; large $>3.5$ or $<0.3$. For Cohen's $d$, we use the usual conventions, but consider $d=0.1$ as "zero/close to zero difference."

Shaded studies are higher quality studies, i.e., with random, representative national samples and standardized clinical interviews or with death by suicide from death registers. Sample sizes are given for the analytic sample, if given in the paper.

Heavy drinking or binge drinking: if not specified otherwise, refers to having $\geq 5$ alcoholic drinks in a row (in one sitting, on one occasion, in a short time).

Some studies are dated 2015 already, because they appeared in print in the meantime, but were electronically available before. 

Abbreviations:

\section{ACASI... Audio Computer Aided Self Interviewing}

ACHA - NCHA ... American College Health Association-National College Health Assessment

AD...Alcohol dependendy disorder

B...Bisexual

BRFSS...Behavioral Risk Factor Surveillance System

CASI...Computer aided self-interview

DD...Drug dependency disorder

DSA... Deliberate Self Harm

G...Gay

GAD...Generalized Anxiety Disorder

HET...Heterosexual

HR...Hazard Ratio

L...Lesbian

MD...Major Depression

moHET...mostly/predominantly heterosexual

moHO...mostly/predominantly homosexual

moG...mostly gay/predominantly gay

moL....mostly lesbian/predominantly lesbian

n.a. Not available

nss...Not statistically significant

OCD...Obsessive Compulsive Disorder

OR...Oddsratio

PD...Panic Disorder

PR...Prevalence Ratio

Q...Questioning/unsure of sexual identity

RR...Risk Ratio

SA...Suicide attempt. Some studies used "deliberate self-harm", especially European studies following the WHO definition, see De Leo, Burgis, Bertolote, Kerkhof, \& Bille-Brahe (2006).

SM...Sexual Minority

SUD...Substance use disorder

ss..Statistically significant

SUD...Substance use disorder

YRBS...Youth Risk Behavior Surveys

$+++\ldots$ Studies reporting the results separate for gender and SM subgroups, i.e., at least separate for homosexual and bisexual individuals.

Footnotes:

a...Did not show up in systematic literature search but is relevant.

b...Of studies on the same sample, only this study entered into the estimation of overall effect sizes. This was to avoid double-counting of similar studies.

+ Series of Studies that used the pooled data from 2005 and 2007 and several districts see Mustanski, Van Wagenen, Birkett, Eyster, \& Corliss (2014) 


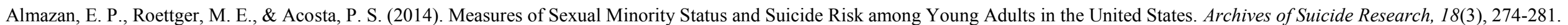

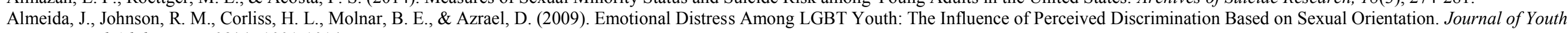
and Adolescence, $38(7), 1001-1014$.

Bagley, C., \& Tremblay, P. (1997). Suicidal behaviors in homosexual and bisexual males. Crisis, 18(1), 24-34

Bagley, C., \& Tremblay, P. (1998). On the prevalence of homosexuality and bisexuality, in a random community survey of 750 men aged 18 to 27 . Journal of Homosexuality, 36(2), 1-18.

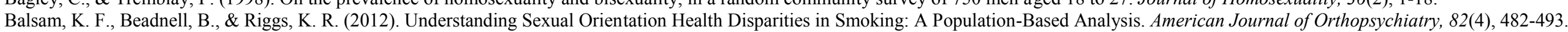

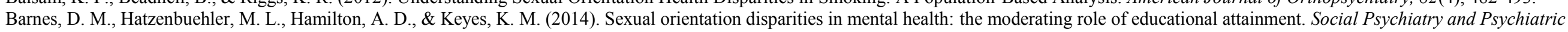
Epidemiology, 49(9), 1447-1454.

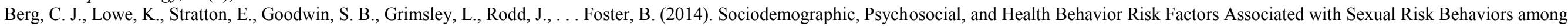
Southeastern US College Students. Open journal of preventive medicine, 4(6), 387-395.

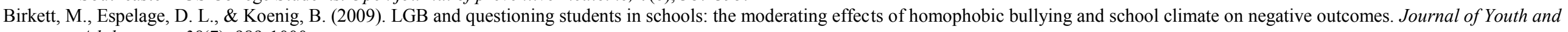
Adolescence, 38(7), 989-1000.

Bloomfield, K. (1993). A comparison of alcohol consumption between lesbians and heterosexual women in an urban population. Drug and alcohol dependence, 33(3), 257-269.

Bloomfield, K., Wicki, M., Wilsnack, S., Hughes, T., \& Gmel, G. (2011). International differences in alcohol use according to sexual orientation. Substance abuse, 32(4), 210-219.

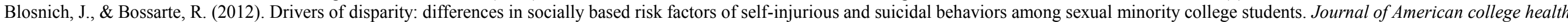
J of $A C H, 60(2), 141-149$.

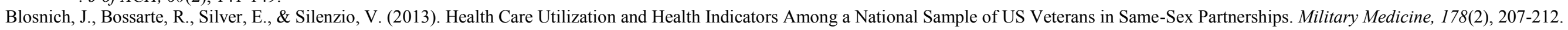
Blosnich, J., Foynes, M. M., \& Shipherd, J. C. (2013). Health Disparities Among Sexual Minority Women Veterans. Journal of Womens Health, 22(7), 631-636.

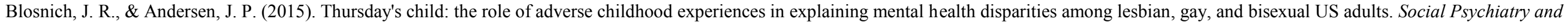
Psychiatric Epidemiology, 50(2), 335-338.

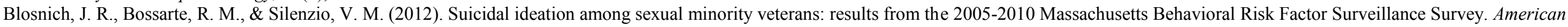
journal of public health, 102 Suppl 1, S44-47.

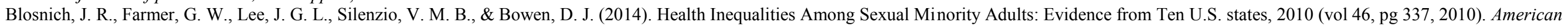
Journal of Preventive Medicine, 47(1), 103-103.

Blosnich, J. R., Mays, V. M., \& Cochran, S. D. (2014). Suicidality Among Veterans: Implications of Sexual Minority Status. American journal of public health, 104, S535-S537.

Blosnich, J. R., \& Silenzio, V. M. B. (2013). Physical health indicators among lesbian, gay, and bisexual US veterans. Annals of Epidemiology, 23(7), 448-451.

Boehmer, U., Miao, X., Linkletter, C., \& Clark, M. A. (2012). Adult health behaviors over the life course by sexual orientation. American journal of public health, 102(2), 292-300.

Boehmer, U., Miao, X., \& Ozonoff, A. (2012). Health behaviors of cancer survivors of different sexual orientations. Cancer causes \& control : CCC, 23(9), 1489-1496.

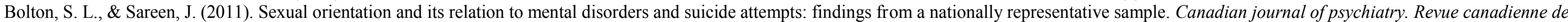
psychiatrie, 56(1), 35-43.

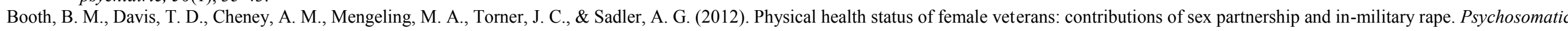
medicine, 74(9), 916-924.

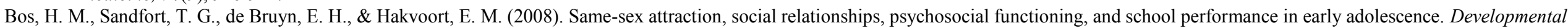
psychology, 44(1), 59-68.

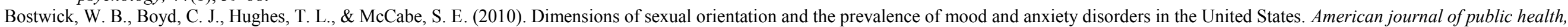
$100(3), 468-475$.

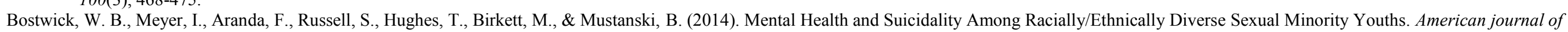
public health, 104(6), 1129-1136.

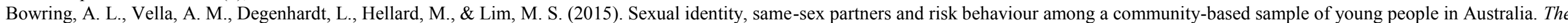
International journal on drug policy, 26(2), 153-161.

Breaugh, J. A. (2003). Effect size estimation: Factors to consider and mistakes to avoid. Journal of Management, 29(1), 79-97.

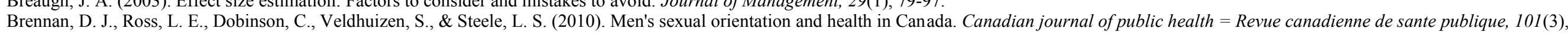
255-258.

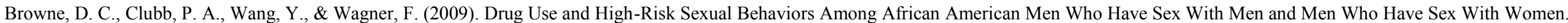
American journal of public health, 99(6), 1062-1066.

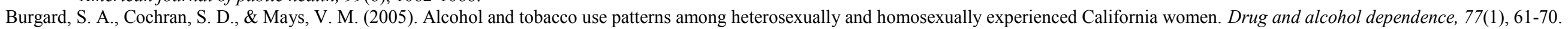

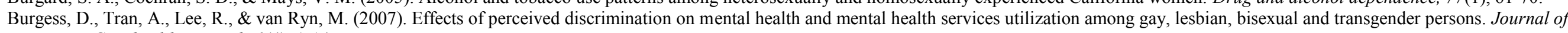
LGBT health research, 3(4), 1-14. 
Online supplemental material to Plöderl \& Tremblay (2015). Mental health of sexual minorities. A systematic review. International Review of Psychiatry. http://dx.doi.org/10.3109/09540261.2015.1083949

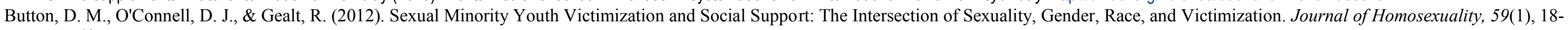
43.

Cardom, R., Rostosky, S., \& Danner, F. (2013). Does "It Get Better" for Depressed Sexual Minority Youth in Young Adulthood? Journal of Adolescent Health, 53(5), 671-673.

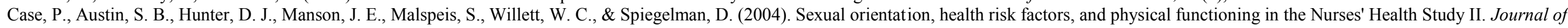
women's health, 13(9), 1033-1047.

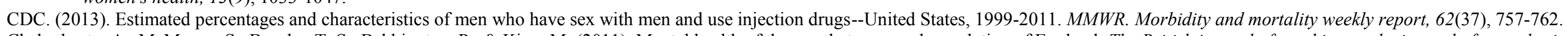

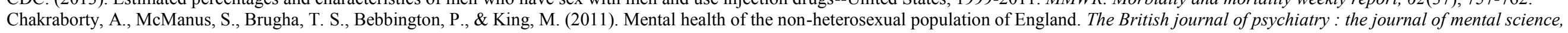
$198(2), 143-148$.

Cheng, C. H., Gipson, J. D., Perez, T. L., \& Cochran, S. D. (2014). Same-Sex Behavior and Health Indicators of Sexually Experienced Filipino Young Adults. Archives of Sexual Behavior.

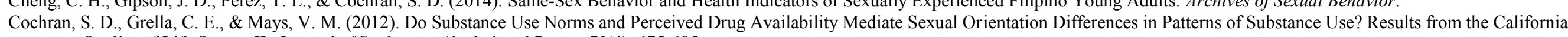
Quality of Life Survey II. Journal of Studies on Alcohol and Drugs, 73(4), 675-685.

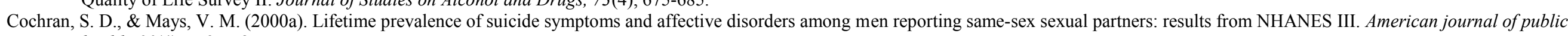
health, 90(4), 573-578.

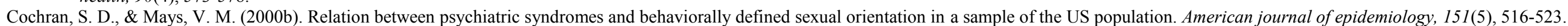

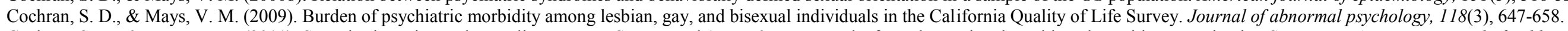

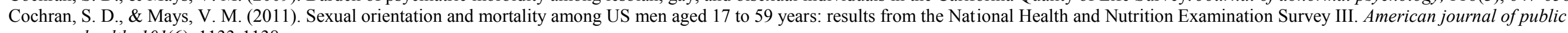
health, 101(6), 1133-1138.

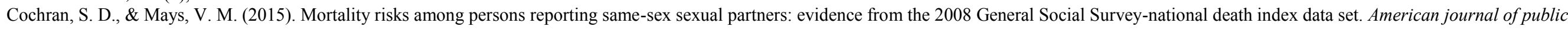
health, 105(2), 358-364.

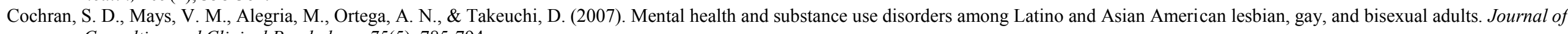
Consulting and Clinical Psychology, 75(5), 785-794.

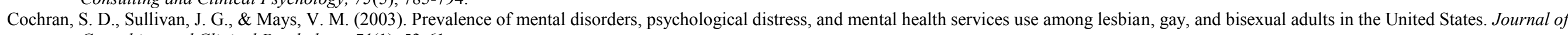
Consulting and Clinical Psychology, 71(1), 53-61.

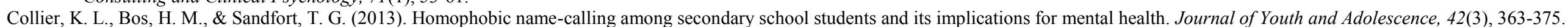

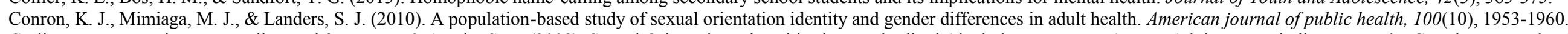

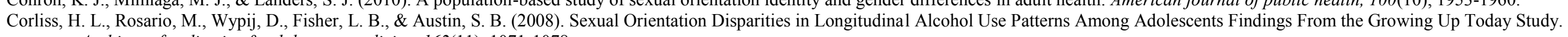
Archives of pediatrics \& adolescent medicine, 162(11), 1071-1078.

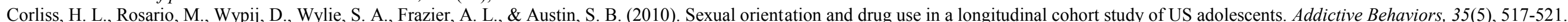

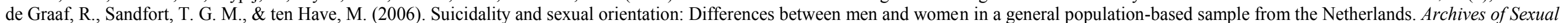
Behavior, 35(3), 253-262.

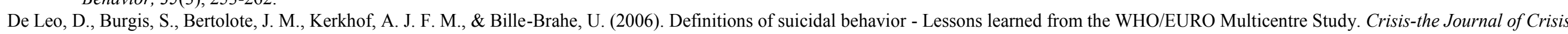
Intervention and Suicide Prevention, 27(1), 4-15.

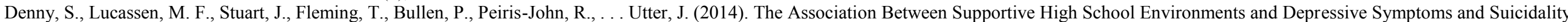
Among Sexual Minority Students. Journal of clinical child and adolescent psychology: the official journal for the Society of Clinical Child and Adolescent Psychology, American Psychological Association, Division 53, 1-14.

Diamant, A. L., \& Wold, C. (2003). Sexual orientation and variation in physical and mental health status among women. Journal of women's health, 12(1), 41-49.

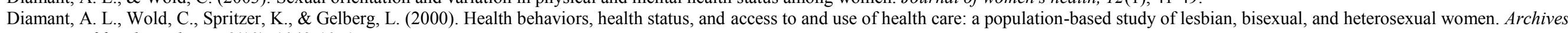
of family medicine, 9(10), 1043-1051.

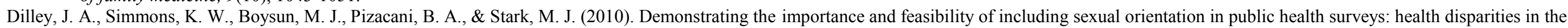
Pacific Northwest. American journal of public health, 100(3), 460-467.

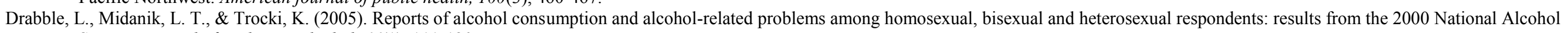
Survey. Journal of studies on alcohol, 66(1), 111-120.

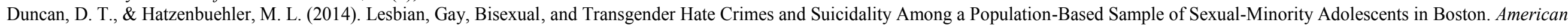
journal of public health, 104(2), 272-278.

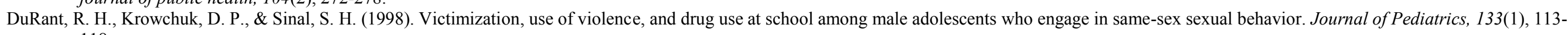
118.

Duryea, D. G., \& Frantz, T. T. (2011). An examination of drinkers' consequences by sexual orientation. Journal of American college health : J of ACH, 59(7), 649-654.

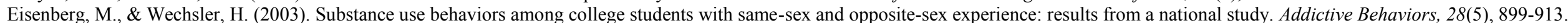

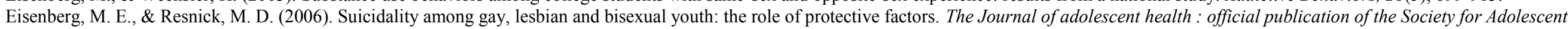
Medicine, 39(5), 662-668. 
Ericksen, K. P., \& Trocki, K. F. (1994). Sex, Alcohol and Sexually-Transmitted Diseases - a National Survey. Family Planning Perspectives, 26(6), $257-263$.

Everett, B., \& Mollborn, S. (2013). Differences in Hypertension by Sexual Orientation Among US Young Adults. Journal of Community Health, $38(3)$, $588-596$.

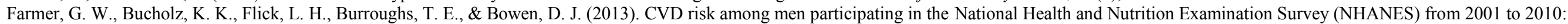
differences by sexual minority status. Journal of epidemiology and community health, 67(9), 772-778.

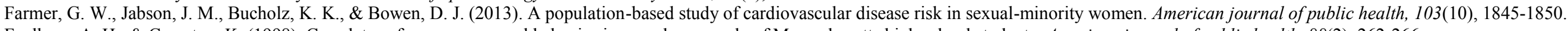

Faulkner, A. H., \& Cranston, K. (1998). Correlates of same-sex sexual behavior in a random sample of Massachusetts high school students. American journal of public health, 88(2), 262-266.

Ferguson, C. J. (2009). An Effect Size Primer: A Guide for Clinicians and Researchers. Professional Psychology-Research and Practice, 40(5), 532-538.

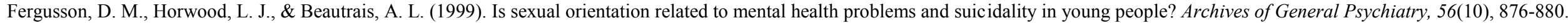

Fergusson, D. M., Horwood, L. J., Ridder, E. M., \& Beautrais, A. L. (2005). Sexual orientation and mental health in a birth cohort of young adults. Psychological Medicine, 35(7), 971-981.

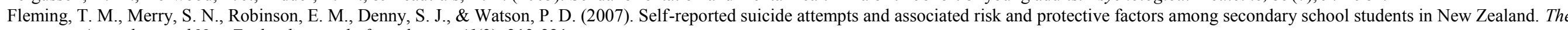
Australian and New Zealand journal of psychiatry, 41(3), 213-221.

Ford, J. A., \& Jasinski, J. L. (2006). Sexual orientation and substance use among college students. Addictive Behaviors, 31(3), 404-413.

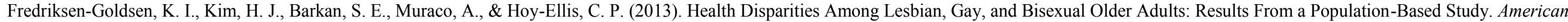
journal of public health, 103(10), 1802-1809.

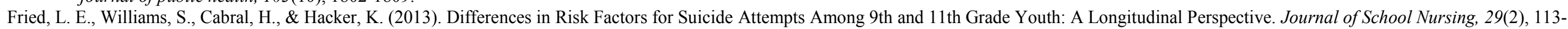
122.

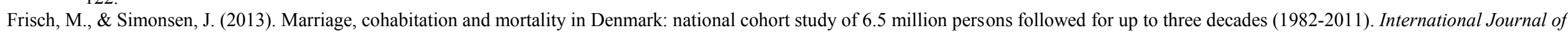
Epidemiology, 42(2), 559-578.

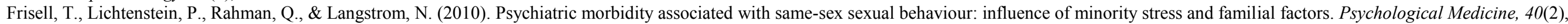
315-324.

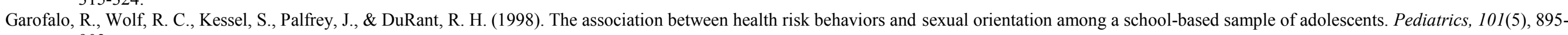
902.

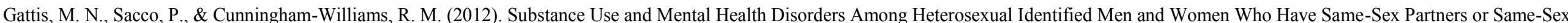
Attraction: Results from the National Epidemiological Survey on Alcohol and Related Conditions. Archives of sexual behavior, 41(5), $1185-1197$.

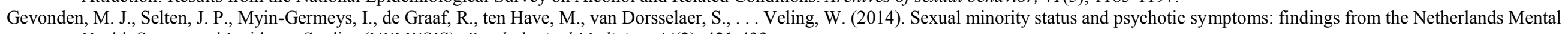
Health Survey and Incidence Studies (NEMESIS). Psychological Medicine, 44(2), 421-433.

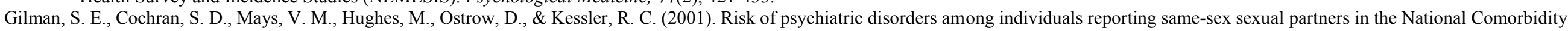
Survey. American journal of public health, 91(6), 933-939.

Gonzales, G., \& Henning-Smith, C. (2015). Disparities in health and disability among older adults in same-sex cohabiting relationships. Journal of aging and health, 27(3), 432-453.

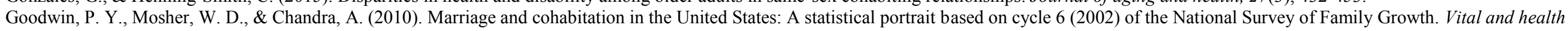
statistics. Series 23, Data from the National Survey of Family Growth(28), 1-45.

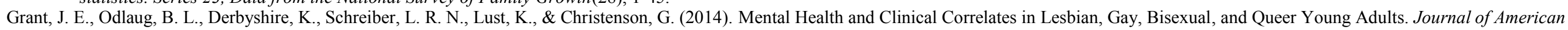
College Health, 62(1), 75-78.

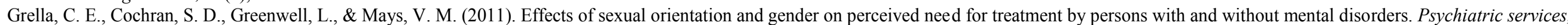
62(4), 404-410

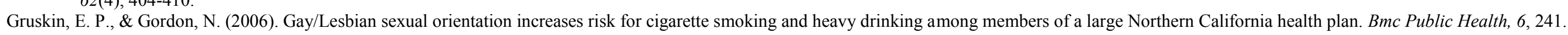

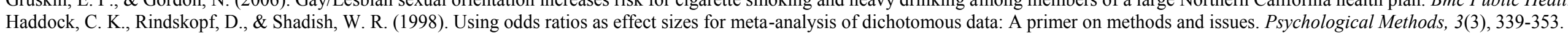

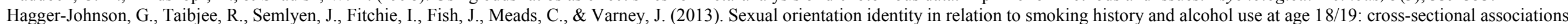
from the Longitudinal Study of Young People in England (LSYPE). BMJ open, 3(8), e002810.

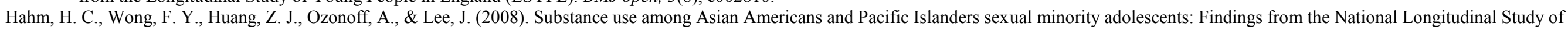
Adolescent Health. Journal of Adolescent Health, 42(3), 275-283.

Hatzenbuehler, M. L. (2011). The social environment and suicide attempts in lesbian, gay, and bisexual youth. Pediatrics, 127(5), 896-903.

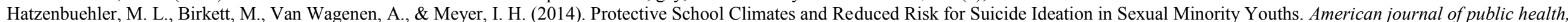
104(2), 279-286.

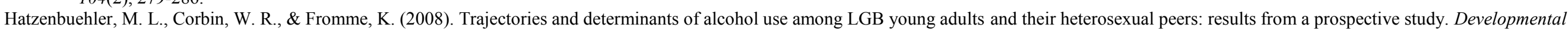
psychology, 44(1), 81-90.

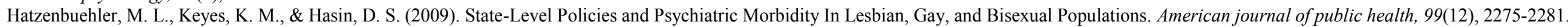

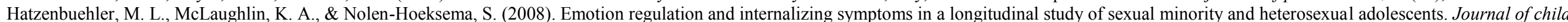
psychology and psychiatry, and allied disciplines, 49(12), 1270-1278.

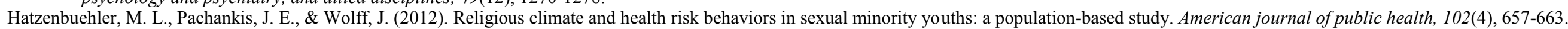


Online supplemental material to Plöderl \& Tremblay (2015). Mental health of sexual minorities. A systematic review. International Review of Psychiatry. http://dx.doi.org/10.3109/09540261.2015.1083949

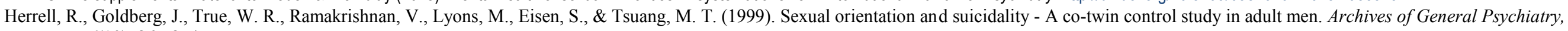
$56(10), 867-874$.

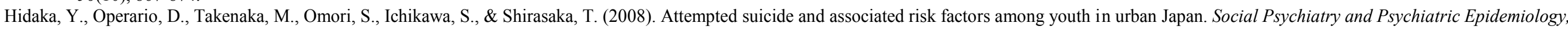
43(9), $752-757$

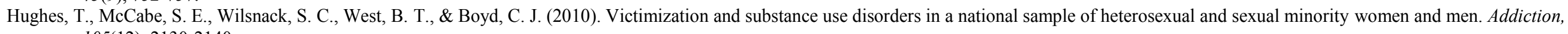
105(12), 2130-2140.

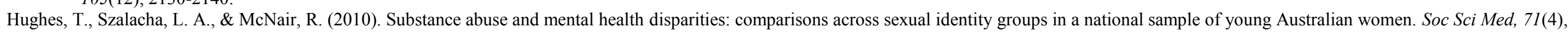
$824-831$.

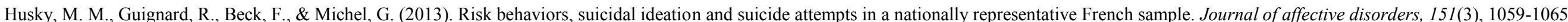

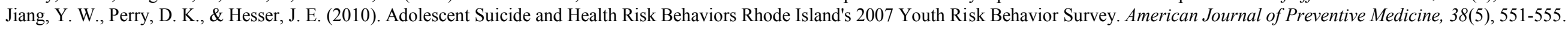

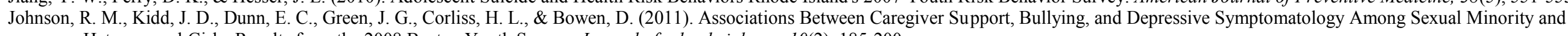
Heterosexual Girls: Results from the 2008 Boston Youth Survey. Journal of school violence, 10(2), 185-200.

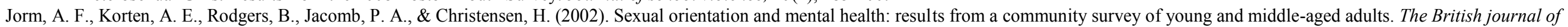
psychiatry: the journal of mental science, 180, 423-427.

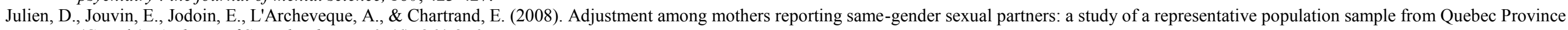
(Canada). Archives of Sexual Behavior, 37(6), 864-876.

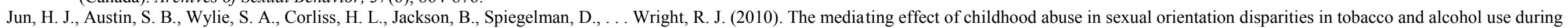
adolescence: results from the Nurses' Health Study II. Cancer causes \& control : CCC, 21(11), 1817-1828.

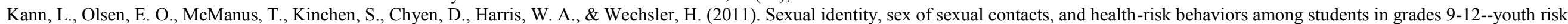
behavior surveillance, selected sites, United States, 2001-2009. Morbidity and mortality weekly report. Surveillance summaries, 60(7), 1-133.

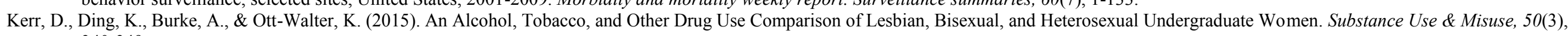
340-349.

Kerr, D. L., Ding, K. L., \& Chaya, J. (2014). Substance Use of Lesbian, Gay, Bisexual and Heterosexual College Students. American Journal of Health Behavior, $38(6)$, 951-962.

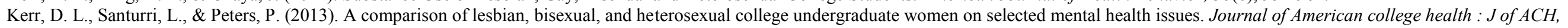
61(4), 185-194.

King, M., \& Nazareth, I. (2006). The health of people classified as lesbian, gay and bisexual attending family practitioners in London: a controlled study. Bmc Public Health, 6, 127.

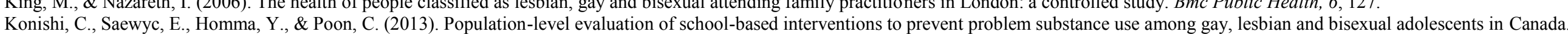
Preventive Medicine, 57(6), 929-933.

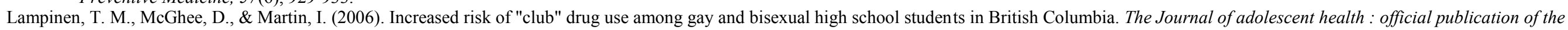
Society for Adolescent Medicine, 38(4), 458-461.

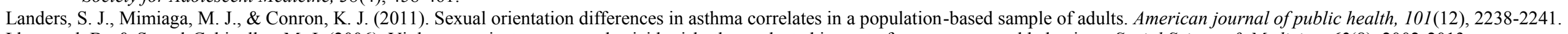

Lhomond, B., \& Saurel-Cubizolles, M. J. (2006). Violence against women and suicide risk: the neglected impact of same-sex sexual behaviour. Social Science \& Medicine, 62(8), 2002-2013.

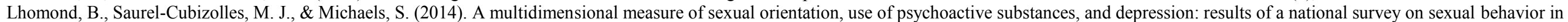
france. Archives of Sexual Behavior, 43(3), 607-619.

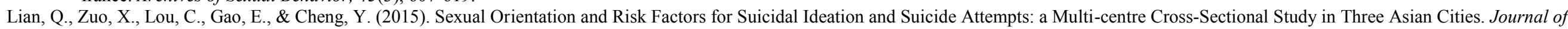
epidemiology / Japan Epidemiological Association, 25(2), 155-161.

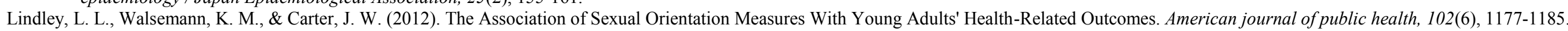

Loosier, P. S., \& Dittus, P. J. (2010). Group Differences in Risk Across Three Domains Using an Expanded Measure of Sexual Orientation. Journal of Primary Prevention, 31(5-6), 261-272.

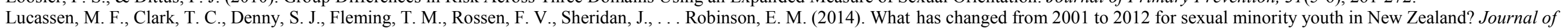
paediatrics and child health.

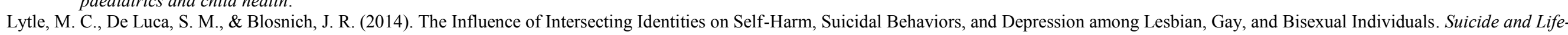
Threatening Behavior, 44(4), 384-391.

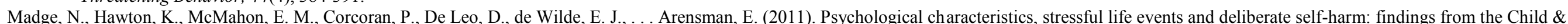
Adolescent Self-harm in Europe (CASE) Study. European child \& adolescent psychiatry, 20(10), 499-508.

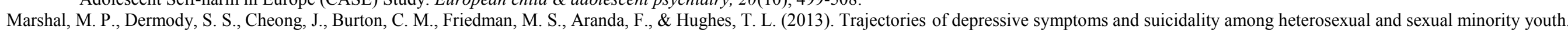
Journal of youth and adolescence, 42(8), 1243-1256.

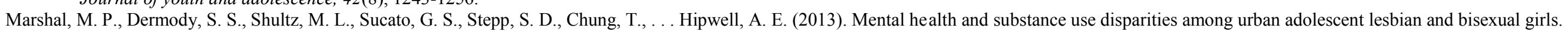
Journal of the American Psychiatric Nurses Association, 19(5), 271-279.

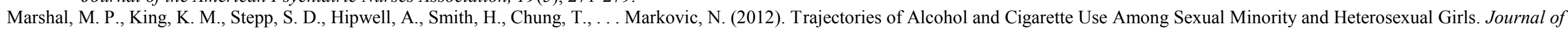
Adolescent Health, 50(1), 97-99. 
Online supplemental material to Plöderl \& Tremblay (2015). Mental health of sexual minorities. A systematic review. International Review of Psychiatry. http://dx.doi.org/10.3109/09540261.2015.1083949

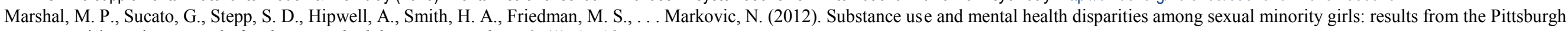
girls study. Journal of pediatric and adolescent gynecology, 25(1), 15-18.

Martin-Storey, A., \& Crosnoe, R. (2012). Sexual minority status, peer harassment, and adolescent depression. Journal of Adolescence, 35(4), 1001-1011.

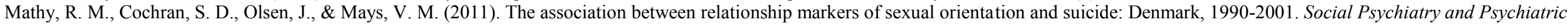
Epidemiology, 46(2), 111-117.

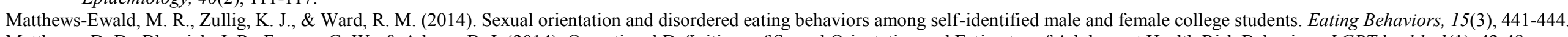

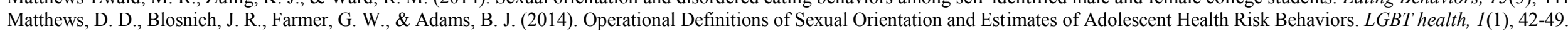

Matthews, D. D., \& Lee, J. G. L. (2014). A Profile of North Carolina Lesbian, Gay, and Bisexual Health Disparities, 2011. American journal of public health, 104(6), E98-E105.

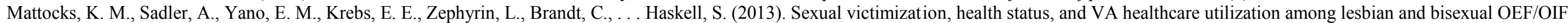
veterans. Journal of general internal medicine, 28 Suppl 2, S604-608.

McCabe, S. E., Boyd, C., Hughes, T. L., \& d'Arcy, H. (2003). Sexual identity and substance use among undergraduate students. Substance abuse, 24(2), 77-91.

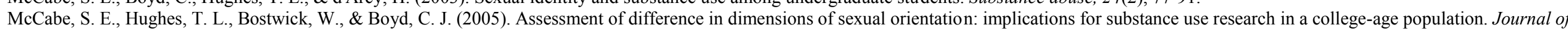
studies on alcohol, 66(5), 620-629.

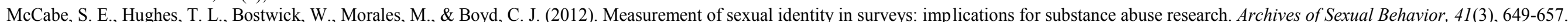

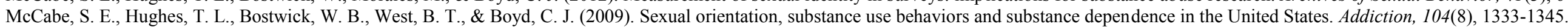

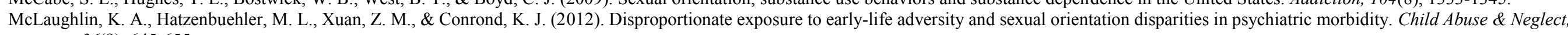
36(9), 645-655.

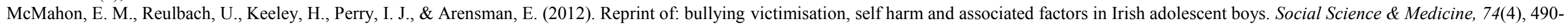
497.

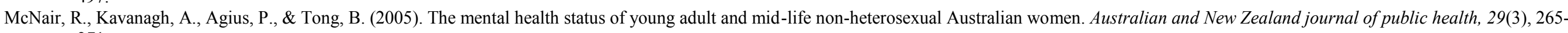
271.

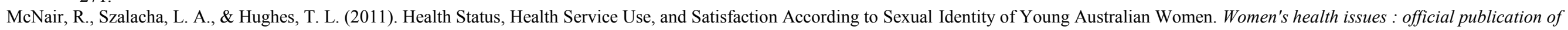
the Jacobs Institute of Women's Health, 21(1), 40-47.

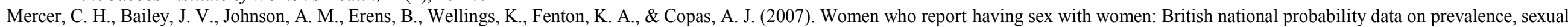
behaviors, and health outcomes. American journal of public health, 97(6), 1126-1133.

Midanik, L. T., Drabble, L., Trocki, K., \& Sell, R. L. (2007). Sexual orientation and alcohol use: identity versus behavior measures. Journal of LGBT health research, 3(1), 25-35.

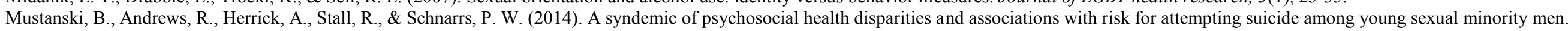
American journal of public health, 104(2), 287-294.

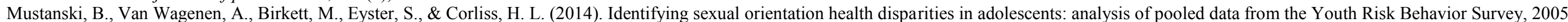
and 2007. American journal of public health, 104(2), 211-217.

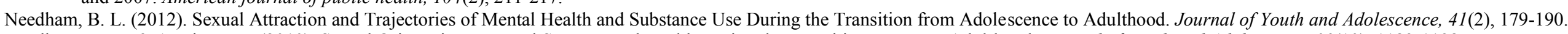

Needham, B. L., \& Austin, E. L. (2010). Sexual Orientation, Parental Support, and Health During the Transition to Young Adulthood. Journal of Youth and Adolescence, 39(10), 1189-1198.

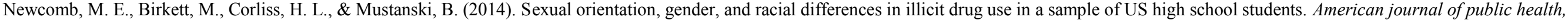
104(2), 304-310.

O'Connor, R. C., Rasmussen, S., \& Hawton, K. (2009). Predicting deliberate self-harm in adolescents: a six month prospective study. Suicide \& life-threatening behavior, 39(4), 364-375.

O'Connor, R. C., Rasmussen, S., \& Hawton, K. (2014). Adolescent self-harm: a school-based study in Northern Ireland. Journal of affective disorders, 159 , $46-52$.

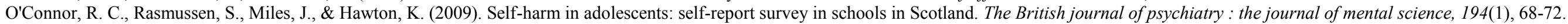

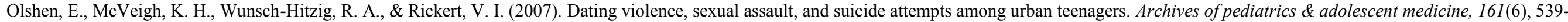
545.

Orenstein, A. (2001). Substance use among gay and lesbian adolescents. Journal of Homosexuality, 41(2), 1-15.

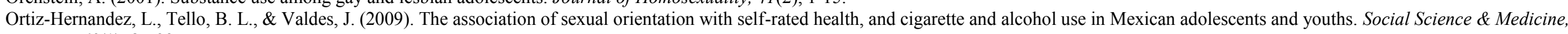
69(1), 85-93.

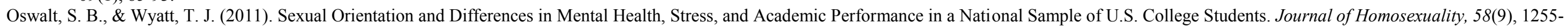
1280.

Pakula, B., \& Shoveller, J. A. (2013). Sexual orientation and self-reported mood disorder diagnosis among Canadian adults. Bmc Public Health, 13.

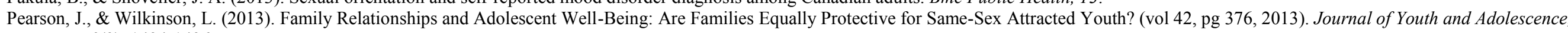
42(9), 1494-1496.

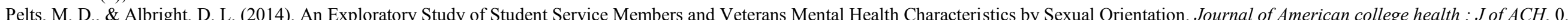

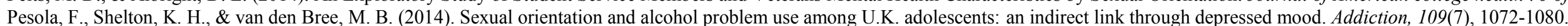

Pinhey, T. K., \& Millman, S. R. (2004). Asian/Pacific Islander adolescent sexual orientation and suicide risk in Guam. American journal of public health, 94(7), 1204-1206. 


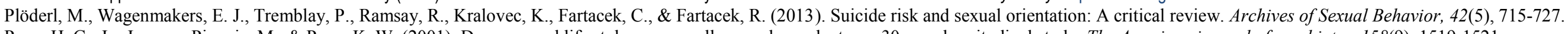

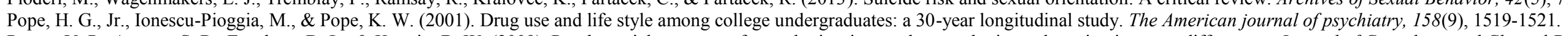

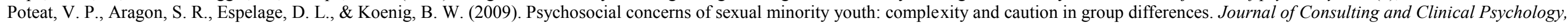
$77(1), 196-201$

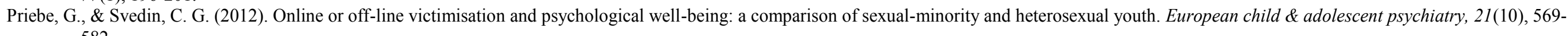
582.

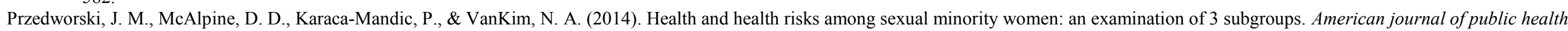
104(6), 1045-1047.

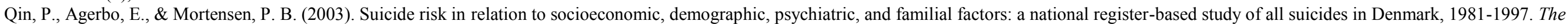
American journal of psychiatry, 160(4), 765-772.

Ramsay, R., \& Tremblay, P. (2015). Gay, Lesbian, Bisexual \& Transgender "Attempted Suicide" Incidences/Risks. Suicidality Studies From 1970 to 2015 Retrieved April, 13, 2015, from http://people.ucalgary.ca/ ramsay/attempted-suicide-gay-lesbian-all-studies.htm\#All-Studies

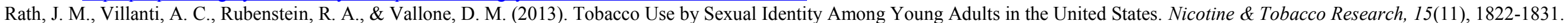

Reczek, C., Liu, H., \& Spiker, R. (2014). A Population-Based Study of Alcohol Use in Same-Sex and Different-Sex Unions. Journal of Marriage and Family, 76(3), 557-572.

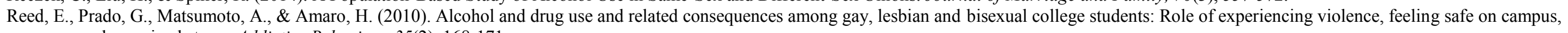
and perceived stress. Addictive Behaviors, 35(2), 168-171.

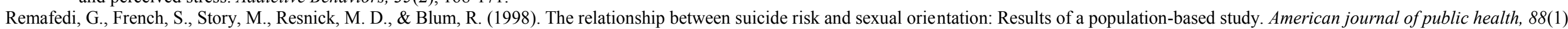
$57-60$.

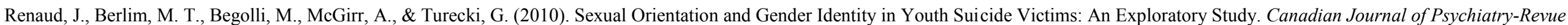
Canadienne De Psychiatrie, 55(1), 29-34.

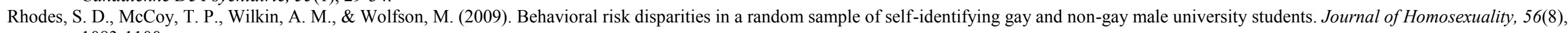
$1083-1100$.

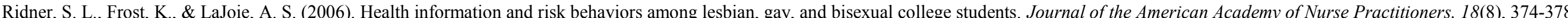

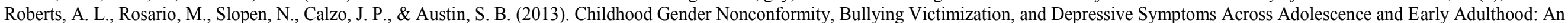
11-Year Longitudinal Study. Journal of the American Academy of Child and Adolescent Psychiatry, 52(2), 143-152.

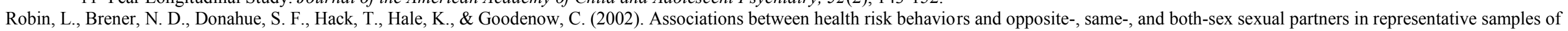
Vermont and Massachusetts high school students. Archives of pediatrics \& adolescent medicine, 156(4), 349-355.

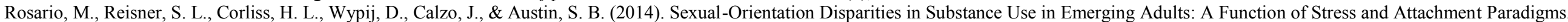
Psychology of Addictive Behaviors, 28(3), 790-804.

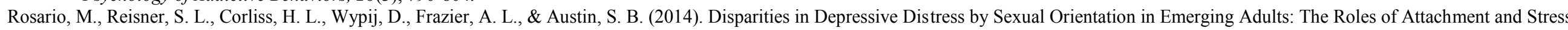
Paradigms. Archives of Sexual Behavior, 43(5), 901-916.

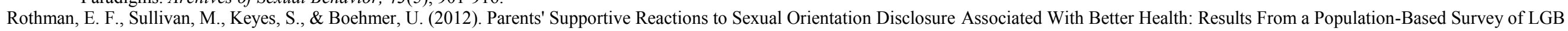
Adults in Massachusetts. Journal of Homosexuality, 59(2), 186-200.

Russell, S. T., \& Joyner, K. (2001). Adolescent sexual orientation and suicide risk: Evidence from a national study. American journal of public health, 91(8), 1276-1281

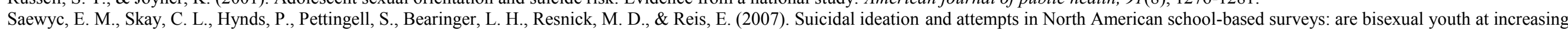
risk? Journal of LGBT health research, 3(2), 25-36.

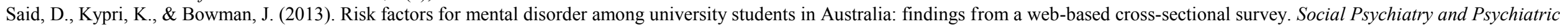
Epidemiology, 48(6), 935-944.

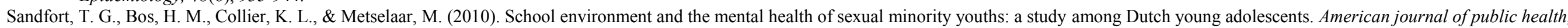
100(9), 1696-1700.

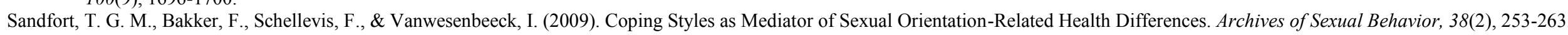

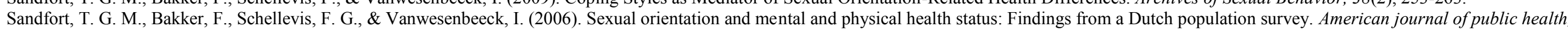
96(6), 1119-1125.

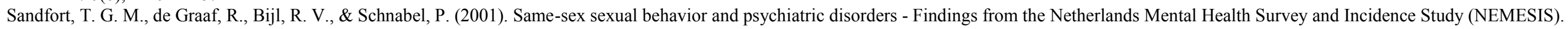
Archives of General Psychiatry, 58(1), 85-91.

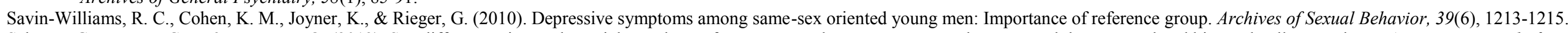

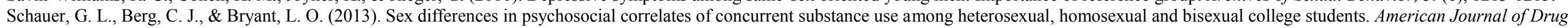
and Alcohol Abuse, 39(4), 252-258.

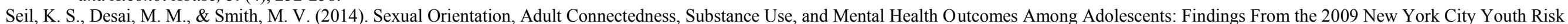
Behavior Survey. American journal of public health, 104(10), 1950-1956. 
Shaffer, D., Fisher, P., Hicks, R. H., Parides, M., \& Gould, M. (1995). Sexual Orientation in Adolescents Who Commit Suicide. Suicide and Life-Threatening Behavior, $25,64-71$.

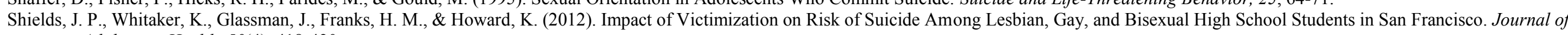
Adolescent Health, 50(4), 418-420.

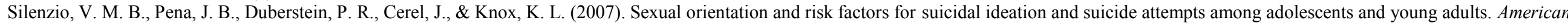
journal of public health, 97(11), 2017-2019.

Skegg, K., Nada-Raja, S., Dickson, N., Paul, C., \& Williams, S. (2003). Sexual orientation and self-harm in men and women. American Journal of Psychiatry, 160(3), 541-546.

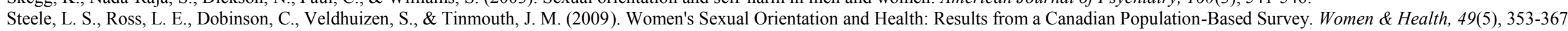

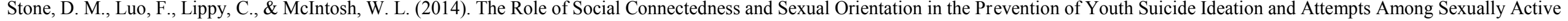
Adolescents. Suicide \& life-threatening behavior.

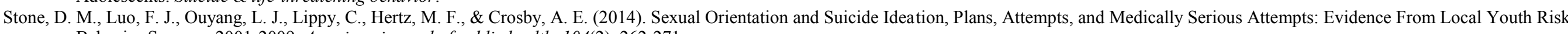
Behavior Surveys, 2001-2009. American journal of public health, 104(2), 262-271.

Strutz, K. L., Herring, A. H., \& Halpern, C. T. (2015). Health Disparities Among Young Adult Sexual Minorities in the US. American Journal of Preventive Medicine, 48(1), 76-88.

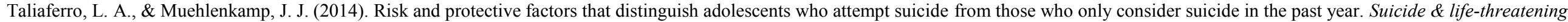
behavior, 44(1), 6-22.

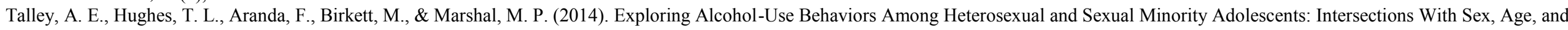
Race/Ethnicity. American journal of public health, 104(2), 295-303.

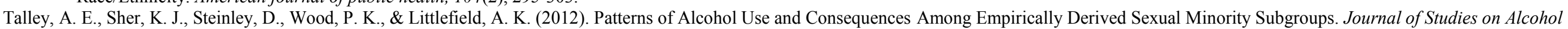
and Drugs, 73(2), 290-302.

Teasdale, B., \& Bradley-Engen, M. S. (2010). Adolescent Same-Sex Attraction and Mental Health: The Role of Stress and Support. Journal of Homosexuality, 57(2), 287-309.

Tjepkema, M. (2008). Health care use among gay, lesbian and bisexual Canadians. Health reports, 19(1), 53-64.

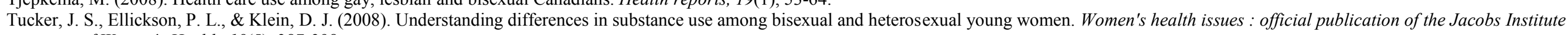
of Women's Health, 18(5), 387-398.

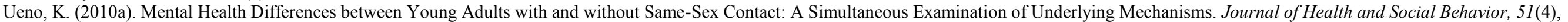
$391-407$.

Ueno, K. (2010b). Same-Sex Experience and Mental Health during the Transition between Adolescence and Young Adulthood. Sociological Quarterly, 51(3), 484-510.

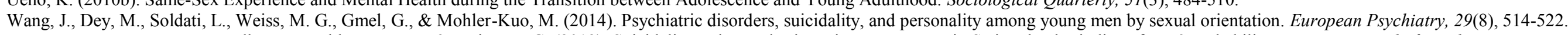

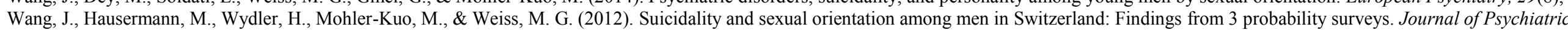
Research, 46(8), 980-986.

Wichstrom, L., \& Hegna, K. (2003). Sexual orientation and suicide attempt: A longitudinal study of the general Norwegian adolescent population. Journal of abnormal psychology, 112(1), 144-151.

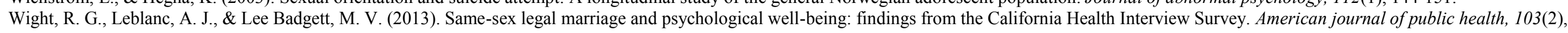
339-346.

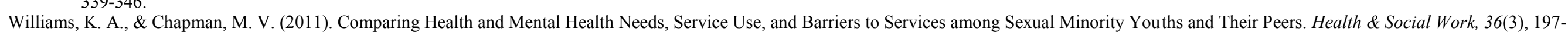
206.

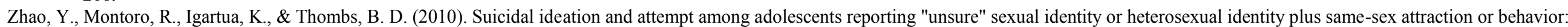
forgotten groups? Journal of the American Academy of Child and Adolescent Psychiatry, 49(2), 104-113.

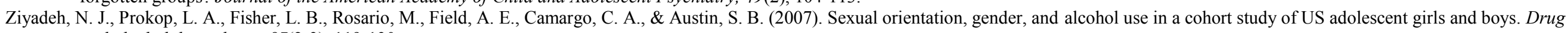
and alcohol dependence, $87(2-3), 119-130$. 